Pure Appl. Chem., Vol. 83, No. 4, pp. 931-1014, 2011.

doi:10.1351/PAC-REC-09-09-36

(C) 2011 IUPAC, Publication date (Web): 14 March 2011

\title{
Glossary of terms used in photocatalysis and radiation catalysis (IUPAC Recommendations 2011)*
}

\author{
Silvia E. Braslavsky ${ }^{1, \ddagger}$, André M. Braun², Alberto E. Cassano ${ }^{3}$, \\ Alexei V. Emeline ${ }^{4}$, Marta I. Litter ${ }^{5}$, Leonardo Palmisano ${ }^{6}$, \\ Valentin N. Parmon ${ }^{7}$, and Nick Serpone ${ }^{8, \star \star}$ \\ ${ }^{1}$ Max Planck Institute for Bioinorganic Chemistry (formerly für Strahlenchemie), \\ Mülheim/Ruhr, Germany; ${ }^{2}$ University of Karlsruhe, Karlsruhe, Germany; \\ ${ }^{3}$ Universidad Nacional del Litoral, Santa Fe, Argentina; ${ }^{4}$ V.A. Fock Institute of \\ Physics, St. Petersburg State University, St. Petersburg, Russia; ${ }^{5}$ National Atomic \\ Energy Commission, Buenos Aires, Argentina; ${ }^{6}$ University of Palermo, Palermo, \\ Italy; ${ }^{7}$ Boreskov Institute of Catalysis, Novosibirsk, Russia; ${ }^{8}$ Concordia University, \\ Montreal, Canada and University of Pavia, Pavia, Italy
}

\begin{abstract}
This glossary of terms covers phenomena considered under the very wide terms photocatalysis and radiation catalysis. A clear distinction is made between phenomena related to either photochemistry and photocatalysis or radiation chemistry and radiation catalysis. The term "radiation" is used here as embracing electromagnetic radiation of all wavelengths, but in general excluding fast-moving particles. Consistent definitions are given of terms in the areas mentioned above, as well as definitions of the most important parameters used for the quantitative description of the phenomena. Terms related to the up-scaling of photocatalytic processes for industrial applications have been included. This Glossary should be used together with the Glossary of terms used in photochemistry, $3^{\text {rd }}$ edition, IUPAC Recommendations 2006: (doi:10.1351/pac200779030293) as well as with the IUPAC Compendium of Chemical Terminology, $2^{\text {nd }}$ ed. (the "Gold Book" 2006-; doi:10.1351/goldbook), because many terms used in photocatalysis are defined in these documents.
\end{abstract}

Keywords: catalysis; glossary; IUPAC Organic and Biomolecular Chemistry Division; IUPAC Physical and Biophysical Chemistry Division; photocatalysis; photochemistry; radiation; radiative transfer; radiocatalysis.

\section{CONTENTS}

\section{INTRODUCTION}

Preliminary issues

Specific problems related to heterogeneous photocatalysis

Radiative transfer equation

Considerations on the evaluation of quantum yields of heterogeneous photocatalysed reactions

A note on the relationship between spectral, radiometric, and photon quantities

\footnotetext{
*Sponsoring bodies: IUPAC Physical and Biophysical Chemistry Division and IUPAC Organic and Biomolecular Chemistry Division: see more details on p. 1011.

¥Corresponding author: E-mail: braslavskys@mpi-muelheim.mpg.de

**For additional members of the working party and collaborators, see p. 1012.
} 


\author{
ALPHABETIC LIST OF TERMS AND DEFINITIONS \\ QUANTITIES, SYMBOLS, AND TERMS USED IN THIS DOCUMENT \\ MEMBERSHIP OF SPONSORING BODIES \\ REFERENCES
}

\title{
INTRODUCTION
}

\section{Preliminary issues}

Studies on environmental photochemistry and natural photosynthesis [1,2], as well as chemical methods for solar energy transformation, involve a variety of phenomena related to both photochemistry and catalysis. Many of these phenomena differ qualitatively and are informally located in different fields of chemistry. Thus, different terms describe similar phenomena in different working areas. It is possible to find in the literature terms such as catalysis of photochemical reactions, photoactivation of catalysts, photochemical activation of catalytic processes, etc. This situation suggested strongly that preparation of a glossary of terms was a desirable project. The resulting Glossary is based on an analysis of terminology associated with both photochemistry and catalysis. The present Glossary collects a set of terms with the aim of harmonising the language of photocatalysis and radiation catalysis. We make the remark that we use the term "radiation" as embracing electromagnetic radiation of all wavelengths but NOT fast-moving particles. The list we present is not exhaustive, but contains crucial terms in the field. The use of the terms recommended in this Glossary should facilitate the communication and the correct understanding of papers published by scientists involved in different research fields.

The term photocatalysis denotes either catalytic reactions proceeding under the action of light, or the overall phenomena connected both with photochemical and catalytic processes. An overview of some approaches in defining photocatalytic phenomena as well as the difficulties involved in the determination of quantum yields for photocatalysed reactions, very often taking place in microheterogenous media, appeared in various publications [3-6].

In this Glossary, we propose some compromises to accommodate different views, which in general originated in different fields of research. In particular, we have incorporated terms related to the upscaling of photocatalytic reactions to an industrial level, a field developed by chemical engineers.

We have adopted the definition of the terms photocatalysis and photocatalyst given in the third edition of the IUPAC "Glossary of terms used in photochemistry" [7]. Only some key entries of the definitions in the latter are included, whereas in other cases, the reader is referred to [7]. Several entries in [7] have been modified from those included in the $2^{\text {nd }}$ edition of the "Gold Book" and are marked in [7] as revised from Gold Book 2 (revG2). It is thus advisable to use the present Glossary in conjunction with [7]. Several definitions from [7] are included in the on-line edition of the "Gold Book" [8]. A few definitions from [8] are different from the latest definitions in the newest edition of the "Green Book": Quantities, Units and Symbols in Physical Chemistry, $3^{\text {rd }}$ ed. [9]. We have tried to adopt the latest definitions.

Many terms referring to solid-state physics, especially in connection with semiconductors, have been included because they are frequently used in the literature on photocatalysis. Terms are also given describing the phenomenon of radiation catalysis as a process generated by absorption of high-energy (ionising) quanta (such as vacuum UV, X-ray and $\gamma$-quanta, and high-speed $\alpha$ - and $\beta$-particles or protons and other accelerated ions) resulting in a primary nonselective excitation of a catalyst (see, e.g., [10]), although in this area we have not necessarily included all terms related to phenomena originated in nuclear excitation.

The arrangement of entries is alphabetical, and the criterion adopted some years ago by the IUPAC Physical Organic Chemistry Commission has been followed for the type of lettering used. Italicised words in a definition or at the bottom of it indicate a relevant cross-reference, a term in "quo- 
tation marks" indicates that it is NOT defined in this Glossary (see [11]). In addition, an underlined word marks its importance in the respective entry. Synonyms appear in non-bolded roman type immediately below the main term.

The same convention as in the "Glossary of terms used in photochemistry" [7] was adopted regarding the magnitudes implying energy or photons incident on a surface from all directions, i.e., the set of symbols included in the IUPAC "Green Book" [9], and recommended by the International Commission on Illumination [12] as follows: $H_{\mathrm{o}}$ or $F_{\mathrm{o}}$ for fluence, $E_{\mathrm{o}}$ for fluence rate, $H_{\mathrm{p}, \mathrm{o}}$ or $F_{\mathrm{p}, \mathrm{o}}$ for photon fluence, and $E_{\mathrm{p}, \mathrm{o}}$ for photon fluence rate; the letter o as subscript indicates radiation incident from all directions. This has been done primarily to comply with internationally agreed symbols. It is important, however, to avoid confusion with the terms used to designate an amount of energy (or photons) prior to absorption. In these cases, the superscript 0 (zero) is used.

It is expected that some of the definitions provided will be subject to change. All suggestions for improvement and updating of the Glossary are welcome. We are sure that progress of the photocatalytic techniques will require updating of this document in the future.

\section{Specific problems related to heterogeneous photocatalysis}

Several parameters used during studies of photocatalysis have much in common with the same or similar parameters in thermal catalysis, but there are specific problems when used for heterogeneous photocatalysis.

The first of such parameters is turnover and associated terms such as turnover number (TON), turnover rate (TOR), and turnover frequency (TOF). These quantities are satisfactorily determined and understood in homogeneous photocatalysis, whereas they are not easily estimated in heterogeneous photocatalysis $[13,14]$. The actual number of photocatalytically active centres is generally unknown. For this reason, the specific surface area of the catalyst (i.e., per mass of the catalyst) is very often used to characterise the specific activity of the catalyst active centres. The knowledge of the specific surface area allows estimation of the maximum possible number of active sites, provided they are uniformly distributed on the catalyst surface. The situation is more complicated in heterogeneous photocatalysis, because dispersed and/or porous particles are used and the actual operating surface is not known since some parts are not illuminated as a consequence of internal shading. Thus, the TON, TOF, and TOR values for heterogeneous photocatalysis usually appear as virtual parameters unsuitable to characterise the actual properties of the photocatalyst active sites. However, we maintain these terms in the Glossary to stress the links between thermal catalysis and photocatalysis.

An additional problem is that in heterogeneous photocatalysis, the area-related parameters (sometimes called areal parameters) do not characterise the catalyst as they do in heterogeneous catalysis, because the properties of a photocatalyst are not directly proportional to its area. Among other factors, this is because light does not reach every part or particle of the photocatalyst to an equal extent.

The second set of important parameters in photocatalysis is the one characterising the efficiency in substrate conversion. Such efficiency is characterised in photochemistry by the quantum yield, $\Phi[7]$. The definition of $\Phi$ assumes knowledge of the number of photons of a particular wavelength absorbed by the system. In homogeneous photochemistry, this quantity can be measured with great accuracy [15]; however, in heterogeneous photocatalysis one can only easily know the number of photons incident onto the surface of the photocatalyst. This is an upper limit for the actual number of absorbed photons, because the scattering phenomena in general reduce the number of active photons, which can be determined exactly only in a few cases. However, the quantum yield can be rigorously determined for nonporous materials or transparent nanoparticles.

For the above reasons, in addition to the quantum yield, the term photonic yield is convenient in photocatalysis. In this context, the term photonic is thus defined in terms of the incident amount of photons arriving at the internal surface of the irradiation window, rather than in terms of the absorbed amount of photons, with the obvious exception of the cases in which the latter quantity can be deter- 
mined. The qualifying expression "apparent photonic" was used in the past to characterise quantities with respect to incident photons. It is believed that photonic is more appropriate for the case of photocatalysis. However, the qualifying word apparent may be useful for the case of incident ionising radia-

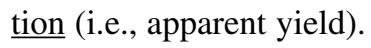

In addition, we have adopted the very generalised use of efficiency when irradiation within a wavelength range is used, whereas the term yield is reserved for excitation with monochromatic photons (i.e., photons of energy in the wavelength range $\lambda$ and $\lambda+\mathrm{d} \lambda$ ) [7]. Thus, quantum yield (similar to homogeneous photochemistry) refers to the number of monochromatic photons absorbed, whereas photonic yield refers to the number of monochromatic photons arriving at the internal surface of the irradiation window.

The prefix "true" when describing quantum yields is misleading and not recommended. The prefix "apparent" should be reserved for data measured or calculated from experimental results obtained under conditions that cannot be necessarily reproduced by others, or for conditions other than those required by the definition of quantum yield or even rate constants, such as, e.g., when quantum yields of reaction of mixtures are determined.

In any case, the term "quantum yield" is strictly valid only for monochromatic irradiation. The use of photonic referred to the number of photons incident on a surface might be considered as violating the "first law of photochemistry", which states that a photon must be absorbed in order to be effective. However, this yield or efficiency (depending on whether the light is monochromatic or polychromatic) may be interpreted as the amount of energy used for a reaction referred to the energy impinging on the surface.

Notwithstanding this fact, the characterisation of the properties of a photocatalyst by means of parameters measured only with monochromatic radiation is not exhaustive. Nonadditive cross-correlations of photocatalytic transformations induced by irradiation by different wavelengths can occur much more likely than in homogeneous photochemistry.

\section{Radiative transfer equation}

The Beer-Lambert equation for radiation absorption [8] is strictly valid only for a homogeneous, isotropic medium, such as a dilute solution, under low radiation fields and for a collimated radiation beam. In the case of scattering systems, such as those encountered in heterogeneous photocatalysis, the law of energy (light) conservation should be carefully applied. The complete radiative transfer equation (RTE) is a way of applying this law for calculations, especially in the design and use of large-scale photocatalytic reactors. This equation explicitly considers the radiation field at any point inside a photoreactor (containing a homogeneous or an inhomogeneous system) in one, two, and three-dimensional spaces. Several terms related to the application of the RTE to dispersive systems have been included in this document.

\section{Considerations on the evaluation of quantum yields of heterogeneously photocatalysed reactions}

(i) It is of fundamental importance to monitor the initial rate of reactant consumption or of product formation. Under these conditions, products do not interfere with the measurements and the possible inactivation of the catalyst is minimised. The quantum yield of substrate consumption, as well as of product formation, generally varies with the irradiation time, especially at longer times.

(ii) The catalyst concentration should not affect the reaction rate.

(iii) Mass transfer limitation must be avoided by effective stirring.

(iv) The radiation energy arriving at any point inside the reactor should be such that the reaction rate depends linearly on the spectral radiance. It is always possible to work under radiation fields of very low intensity, although the experiment might take longer times. 
(v) Monochromatic radiation should be used. However, most often polychromatic radiation is used and the spectra of the incident radiation and of the absorbed substrate must be taken into account. This causes a serious problem.

(vi) The absorbed radiation should be properly determined (either by actinometry or by proper calculation).

(vii) The adsorption of the reactant on the catalyst must have reached steady-state regime before starting irradiation.

(viii) The radiation source should have reached steady-state regime.

(ix) Catalyst accumulation at the windows of the reactor must be carefully avoided.

(x) The catalyst preparation must be carefully described.

\section{A note on the relationship between spectral, radiometric, and photon quantities [7]}

When a quantity $(G)$ expressed in photon units (index p, $G_{\mathrm{p}}$ ) covers a wavelength range (polychromatic irradiation between $\lambda_{1}$ and $\lambda_{2}$ ), then $G_{\mathrm{p}}$ is the integral between $\lambda_{1}$ and $\lambda_{2}$ of the corresponding spectral photon quantity, $G_{\mathrm{p}}(\lambda)$ :

$$
G_{\mathrm{p}}=\int_{\lambda_{1}}^{\lambda_{2}} G_{\mathrm{p}}(\lambda) \mathrm{d} \lambda
$$

(e.g., spectral photon flux in $\mathrm{s}^{-1} \cdot \mathrm{nm}^{-1}$ ).

A spectral radiometric or energetic quantity at a given wavelength $\lambda\left(G_{\mathrm{e}, \lambda}\right.$, e.g., spectral radiant power, $\left.P_{\lambda} / \mathrm{W} \mathrm{nm}{ }^{-1}\right)$ is related to the corresponding photon quantity at the same wavelength $\left(G_{\mathrm{p}, \lambda}\right.$, e.g., spectral photon flux in $\mathrm{s}^{-1} \cdot \mathrm{nm}^{-1}$ ) by the relation

$$
G_{\mathrm{e}, \lambda}=E_{\mathrm{p}, \lambda} G_{\mathrm{p}, \lambda}
$$

with $E_{\mathrm{p}, \lambda}=h c / \lambda$, the energy of a photon of wavelength $\lambda$.

The relation between photon $\left(G_{\mathrm{p}}\right)$ and corresponding radiometric (or energetic, $G_{\mathrm{e}}$ ) quantity is thus given by

$$
G_{\mathrm{e}}=h c \int_{\lambda_{1}}^{\lambda_{2}} G_{\mathrm{p}}(\lambda) 1 / \lambda \mathrm{d} \lambda
$$

Or, more useful in practice

$$
G_{\mathrm{p}}(\lambda)=1 /(h c) \int_{\lambda_{1}}^{\lambda_{2}} G_{\mathrm{e}}(\lambda) \lambda \mathrm{d} \lambda
$$

Therefore, for example, to calculate a photon flux over a wavelength interval, the spectral distribution of the radiant power is necessary. Note that in [7] and in the present document no subscript e has been used for the radiometric quantities.

Radiometric quantities ( $G_{\mathrm{e}}$ as above, radiant power and others) are needed because lamp providers usually give the spectral distribution of the lamps in these units, and not in photon units $\left(G_{\mathrm{p}}\right.$, photon flux and other photon quantities) and because of quantification of radiation using, e.g., radiometers.

\section{ALPHABETIC LIST OF TERMS AND DEFINITIONS}

\section{absorbance (decadic $A_{10}$ or napierian $A_{\mathrm{e}}$ )}

See $[7,8]$ for this and related terms.

See attenuance, extinction coefficient, specific (spectral) absorption coefficient.

absorbed local spectral photon flux density, $L_{\mathrm{p}, \lambda}^{\mathrm{a}}(\boldsymbol{x}, t)$

local volumetric rate of photon absorption

local spectral photon density absorption rate 
Local value of the spectral photon radiance, or spectral photon flux density, defined for a three-dimensional space, often in terms of napierian properties.

Note 1: Mathematical expression for one spatial dimension is

$$
L_{\mathrm{p}, \lambda}^{\mathrm{a}}(x, t)=\alpha_{\lambda}(\boldsymbol{x}, t) L_{\mathrm{p}, \lambda}(x, t)
$$

where $L_{\mathrm{p}, \lambda}(x, t)$ is the time-dependent spectral photon radiance incident from one direction at a point defined by the space coordinate $x . \alpha_{\lambda}(\boldsymbol{x}, t)$ is the linear napierian absorption coefficient. SI unit is $\mathrm{s}^{-1} \cdot \mathrm{m}^{-4}$ in photon number basis and $\mathrm{mol} \mathrm{s}-1 \cdot \mathrm{m}^{-4}$ in chemical amount basis. Common unit is $\mathrm{s}^{-1} \cdot \mathrm{cm}^{-3} \cdot \mathrm{nm}^{-1}$ and einstein $\cdot \mathrm{s}^{-1} \cdot \mathrm{cm}^{-3} \cdot \mathrm{nm}^{-1}$, respectively. The above equation is valid for a collimated beam of radiation. In this case,

$$
E_{\mathrm{p}, \lambda, 0}(\boldsymbol{x}, t)=L_{\mathrm{p}, \lambda}(\boldsymbol{x}, t)
$$

with the subindex o (the letter o) indicating radiation from all directions.

Note 2: For a divergent beam, the mathematical definition is: $L_{\mathrm{p}, \lambda}^{\mathrm{a}}(\boldsymbol{x}, t)=\alpha_{\lambda}(\boldsymbol{x}, t) E_{\mathrm{p}, \lambda, \mathrm{o}}(\boldsymbol{x}, t)$, with $\boldsymbol{x}$ a position vector in a three-dimensional space. Units are the same as above. In this case: $E_{\mathrm{p}, \lambda, \mathrm{o}}(\boldsymbol{x}, t)=\int_{4 \pi} L_{\mathrm{p}, \lambda}(\boldsymbol{x}, t) \mathrm{d} \Omega$.

In a three-dimensional space, units of $L_{\mathrm{p}, \lambda}(\boldsymbol{x}, t)$ are $\mathrm{mol} \mathrm{s}{ }^{-1} \cdot \mathrm{m}^{-3} \cdot \mathrm{sr}^{-1}$. Thus, the integral over the solid angle $\Omega$ is needed to obtain $L_{\mathrm{p}, \lambda}^{\mathrm{a}}$ in units $\mathrm{mol} \mathrm{s}^{-1} \cdot \mathrm{m}^{-4}$.

Note 3: Rigorously speaking, the general definition should be written in terms of $E_{\mathrm{p}, \lambda, \mathrm{o}}$. However, for a collimated beam of radiation, it is clearer to write it directly in terms of $L_{\mathrm{p}, \lambda}$.

Note 4: Also called local volumetric rate of photon absorption, LVRPA [either $e_{\lambda}^{\mathrm{a}}(x, t)$ for onedimensional or $e_{\lambda}^{\mathrm{a}}(\boldsymbol{x}, t)$ for three-dimensional absorbed photon flux densities, respectively] in [16].

Note 5: In practice, for polychromatic radiation, the quantity is integrated in the wavelength range used.

\section{absorbed local spectral radiance, $L_{\lambda}^{\mathrm{a}}(\boldsymbol{x}, t)$}

local volumetric rate of energy absorption

Local value of the spectral radiance, or energy density absorption rate, defined for a three-dimensional space, often in terms of napierian properties.

Note 1: Mathematical expression for one spatial dimension is

$$
L_{\lambda}^{\mathrm{a}}(x, t)=\alpha_{\lambda}(\boldsymbol{x}, t) L_{\lambda}(x, t)
$$

where $L_{\lambda}(x, t)$ is the time-dependent spectral radiance incident from one direction at a point $x$ defined in the space coordinates, $\alpha_{\lambda}(x, t)$ is the space- and time-dependent linear napierian absorption coefficient.

SI unit is $\mathrm{W} \mathrm{m}{ }^{-4}$. Common unit is $\mathrm{W} \cdot \mathrm{cm}^{-3} \cdot \mathrm{nm}^{-1}$.

The equation above is valid for a collimated beam of radiation, for which $E_{\lambda, 0}(\boldsymbol{x}, t)=L_{\lambda}(\boldsymbol{x}, t)$.

Note 2: For a divergent beam, the mathematical definition is: $L_{\lambda}^{\mathrm{a}}(\boldsymbol{x}, t)=\alpha_{\lambda}(\boldsymbol{x}, t) E_{\lambda, \mathrm{o}}(\boldsymbol{x}, t)$. In this case, $\boldsymbol{x}$ is a position vector in a three-dimensional space. Units are the same as above.

Note 3: Rigorously speaking, the general definition should be written in terms of $E_{\lambda, \mathrm{o}}$. However, for a collimated beam of radiation, it is clearer to write it directly in terms of $L_{\lambda}$. 
Note 4: Also called local volumetric rate of e energy absorption, LVREA, [either $e_{\lambda}^{\mathrm{a}}(x, t)$ for one spatial dimension or $e_{\lambda}^{\mathrm{a}}(\boldsymbol{x}, t)$ for three dimensions] in [16].

Note 5: In practice, for polychromatic irradiation, the quantity is integrated in the wavelength range used.

absorbed (spectral) photon flux density, $\left\langle L_{\mathrm{p}, \lambda}^{\mathrm{a}}(t)\right\rangle_{V}$

average volumetric rate of photon absorption

absorbed (spectral) photon radiance

Number of photons of a particular wavelength, per time interval (spectral photon radiance) coming from all directions and absorbed by a system, integrated over the whole volume and averaged per volume $V$.

Note 1: Spectral photon flux, photon number basis, $q_{\mathrm{p}, \lambda}$, (or spectral photon flux, chemical amount basis, $\left.q_{n, \mathrm{p}, \lambda}\right)$, coming from all directions and absorbed by a system integrated over the whole volume and averaged per volume $V$.

Note 2: Mathematical expression:

$$
\left\langle L_{\mathrm{p}, \lambda}^{\mathrm{a}}(t)\right\rangle_{V}=\frac{1}{V} \int_{V} L_{\mathrm{p}, \lambda}^{\mathrm{a}}(\boldsymbol{x}, t) \mathrm{d} V=\frac{1}{V} \int_{V} \alpha_{\lambda}(\boldsymbol{x}, t) E_{\mathrm{p}, \lambda, \mathrm{o}}(\boldsymbol{x}, t) \mathrm{d} V
$$

with $x$ a position vector in a three-dimensional space, $V$ the volume of reaction, $E_{\mathrm{p}, \lambda, 0}(\boldsymbol{x}, t)$ the incident from all directions space- and time-dependent spectral photon fluence rate, and $\alpha(\boldsymbol{x}, t)$ the space- and time-dependent linear napierian absorption coefficient (absorption coefficients are always spectral and the subscript $\lambda$ may be omitted).

SI unit in number basis is $\mathrm{s}^{-1} \cdot \mathrm{m}^{-4}$ and in chemical amount basis is $\mathrm{mol} \cdot \mathrm{s}^{-1} \cdot \mathrm{m}^{-4}$. Common unit in photon number basis is $\mathrm{s}^{-1} \cdot \mathrm{cm}^{-3} \cdot \mathrm{nm}^{-1}$ and in chemical amount basis is einstein $\cdot \mathrm{s}^{-1} \cdot \mathrm{cm}^{-3} \cdot \mathrm{nm}^{-1}$. Called $\left\langle e_{\lambda}^{\mathrm{a}}(t)\right\rangle_{V}$ in [16].

Note 3: $\left\langle L_{\mathrm{p}, \lambda}^{\mathrm{a}}(t)\right\rangle_{V}$ is the average over the total volume, $V$, of the absorbed local spectral photon flux density.

Note 4: Absorbed spectral photon flux density, photon number basis or chemical amount basis, should be in the denominator when calculating a differential quantum yield and using in the numerator the rate of change of the number concentration, $\mathrm{d} C / \mathrm{d} t$, or the rate of change of the amount concentration, $\mathrm{d} c / \mathrm{d} t$, respectively. The product concentration will be used to calculate the product quantum yield, whereas the reactant concentration will be used to calculate the reactant disappearance.

Note 5: In practice, for polychromatic irradiation, the quantity is integrated in the wavelength range used.

Note 6: Note 1 in this entry in [7] applies only for homogeneous, nonscattering media.

See [16].

Note 7: Comparative protocols have been proposed to determine absorbed and reflected photon numbers in systems containing powders as photocatalysts and under strictly specified conditions [17].

absorbed (spectral) photon radiance, $\left\langle L_{\mathrm{p}, \lambda}^{\mathrm{a}}(t)\right\rangle_{V}$

absorbed (spectral) photon flux density

average volumetric rate of photon absorption

(C) 2011, IUPAC

Pure Appl. Chem., Vol. 83, No. 4, pp. 931-1014, 2011 
absorbed (spectral) radiant density, $\left\langle L_{\lambda}^{\mathrm{a}}(t)\right\rangle_{V}$

Monochromatic radiant energy, per time interval (spectral radiance) coming from all directions and absorbed by a system integrated over the whole volume and averaged per volume $V,\left\langle L_{\lambda}^{\mathrm{a}}(t)\right\rangle_{V}$.

Note 1: Mathematical expression:

$$
\left\langle L_{\lambda}^{\mathrm{a}}(t)\right\rangle_{V}=\frac{1}{V} \int_{V} L_{\lambda}^{\mathrm{a}}(\boldsymbol{x}, t) \mathrm{d} V=\frac{1}{V} \int_{V} \alpha_{\lambda}(\boldsymbol{x}, t) E_{\lambda, \mathrm{o}}(\boldsymbol{x}, t) \mathrm{d} V
$$

over the absorption volume $V$, with $\boldsymbol{x}$ a position vector in a three-dimensional space, $\alpha(\boldsymbol{x}, t)$ the space- and time-dependent linear napierian absorption coefficient (absorption coefficients are always spectral and the subscript $\lambda$ may be omitted), and $E_{\lambda, 0}(x, t)$ the incident from all directions space- and time-dependent spectral fluence rate. SI unit is $\mathrm{W} \cdot \mathrm{m}^{-4}$. Common unit is $\mathrm{W} \cdot \mathrm{cm}^{-3} \cdot \mathrm{nm}^{-1}$. Called $\left\langle e_{\lambda}^{\mathrm{a}}(t)\right\rangle_{V}$ in [16].

Note 2: In practice, for polychromatic irradiation, the quantity is integrated in the wavelength range used.

absorption (of electromagnetic radiation)

Transfer of energy from an electromagnetic field to a material or a molecular entity [7].

Note 1: The absorption intensity is determined by the interaction of the electromagnetic radiation and the transition dipole moment of the entities in the medium.

Note 2: A term in the radiative transfer equation (RTE) defined as $\alpha_{\lambda}(\boldsymbol{x}, t) L_{\Omega, \lambda}(\boldsymbol{x}, t)$, with $\alpha_{\lambda}(\boldsymbol{x}, t)$ the spectral linear napierian absorption coefficient defined for a point $\boldsymbol{x}$ in a three-dimensional space and $L_{\Omega, \lambda}(\boldsymbol{x}, t)$ the incident spectral radiance. SI unit is $\mathrm{W} \cdot \mathrm{sr}^{-1} \cdot \mathrm{m}^{-4}$. Common unit is $\mathrm{W} \cdot \mathrm{sr}^{-1} \cdot \mathrm{cm}^{-3} \cdot \mathrm{nm}^{-1}$.

Note 3: Mathematical definition in terms of photons: $\alpha_{\lambda}(\boldsymbol{x}, t) L_{\mathrm{p}, \Omega, \lambda}(\boldsymbol{x}, t)$ with $L_{\mathrm{p}, \Omega, \lambda}(x, t)$ the incident spectral photon radiance. SI unit is $\mathrm{s}^{-1} \cdot \mathrm{sr}^{-1} \cdot \mathrm{m}^{-4}$ in photon number basis and $\mathrm{mol} \cdot \mathrm{s}^{-1} \cdot \mathrm{sr}^{-1} \cdot \mathrm{m}^{-4}$ in chemical amount basis. Common unit in photon number basis is $\mathrm{s}^{-1} \cdot \mathrm{sr}^{-1} \cdot \mathrm{cm}^{-3} \cdot \mathrm{nm}^{-1}$ and einstein $\cdot \mathrm{s}^{-1} \cdot \mathrm{sr}^{-1} \cdot \mathrm{cm}^{-3} \cdot \mathrm{nm}^{-1}$ in chemical amount basis.

See [8] for two meanings referred to absorption of matter or of radiation. See also local differential absorption [7].

See attenuance.

absorption coefficient [linear decadic $a(\lambda)$ or linear napierian $\alpha(\lambda)$ ]

Absorbance, $A(\lambda)$, divided by the optical pathlength, $l$ :

$$
a(\lambda)=\frac{A(\lambda)}{l}=\left(\frac{1}{l}\right) \lg \left(\frac{P_{\lambda}^{0}}{P_{\lambda}}\right)
$$

where $P_{\lambda}^{0}$ and $P_{\lambda}$ are, respectively, the incident and transmitted spectral radiant power.

When napierian logarithms are used:

$$
\alpha(\lambda)=\frac{A(\lambda)}{l}=\left(\frac{1}{l}\right) \ln \left(\frac{P_{\lambda}^{0}}{P_{\lambda}}\right)
$$

where $\alpha(\lambda)$ is the linear napierian absorption coefficient. Since absorbance is a dimensionless quantity, the coherent SI unit for $a(\lambda)$ and $\alpha(\lambda)$ is $\mathrm{m}^{-1}$; common unit is $\mathrm{cm}^{-1}$.

See attenuance, extinction coefficient [7]. 


\section{accumulation layer}

Subsurface space where the majority carriers accumulate near the surface of a semiconductor because of band bending caused by the surface potential.

Note: The surface charge is of opposite sign to that of the majority charge carriers in the semiconductor. This leads to accumulation of the majority charge carriers in a subsurface layer.

See band bending, depletion layer, inversion layer.

See, e.g., [18].

\section{actinic}

See [7]. This term and its derived terms (e.g., actinometer) should not be confused with the element actinium and the actinoids.

\section{action spectrum}

Plot of a relative biological or chemical photoresponse per number of incident photons (this means using photonic yields), vs. wavelength, or energy of radiation, or frequency or wavenumber.

Note 1: This form of presentation is frequently used in studies of biological or solid-state systems, where the nature of the absorbing species is unknown. It is advisable to control that the fluence dependence of the photoresponse is the same (e.g., linear) for all the wavelengths studied.

Note 2: The action spectrum is sometimes called spectral responsivity or sensitivity spectrum. The precise action spectrum (e.g., in homogeneous photocatalysis) is a plot of the spectral (photon or quantum) effectiveness [7]. By contrast, a plot of the biological or chemical change or response per absorbed photon (quantum yield in homogeneous medium) vs. wavelength is the efficiency spectrum [7].

Note 3: The definition of quantum efficiency given in Note 2 applies for homogeneous photoreactions but does not correspond to the concepts and terms used in heterogeneous photocatalysis. In the latter case, photonic yield is defined in terms of the amount of incident photons and monochromatic excitation.

Note 4: In cases where the fluence dependence of the photoresponse is not linear (as is often the case in catalysed photoresponses) a plot of the photoresponse vs. fluence should be made at several wavelengths and a standard response should be chosen (e.g., two-lg reduction). A plot of the inverse of the "standard response" level versus wavelength is then the action spectrum of the photoresponse.

See photonic yield spectrum $[7,8]$.

\section{active state of a surface photoadsorption centre}

Electronically excited surface centre, or a surface centre (surface site) with a trapped photogenerated charge carrier that interacts with species at the solid/liquid, liquid/liquid, solid/gas, or liquid/gas interfaces with formation of, e.g., chemisorbed species.

See chemisorption, photoadsorption centre. 


\section{active state of a surface photocatalytic centre}

Electronically excited surface centre, or a surface centre (surface site) with a trapped photogenerated charge carrier or reactive intermediate that interacts with molecular entities at an interface with the formation of reaction products or intermediates.

See photocatalytic centre.

adsorbate (adsorbed species)

Species (molecule, ion, or atom) adsorbed on the surface of a solid due to physical (physisorption) or chemical (chemisorption) interactions between the solid and the molecule.

See $[8,9]$.

\section{adsorption}

Process by which an entity (molecule, atom, or ion) is accumulated at a surface of a solid (or more rarely of a liquid) through either a physical (physisorption) or chemical (chemisorption) interaction between the entity and the surface.

See $[8,9]$.

\section{albedo}

scattering albedo

apparent yield (in radiation catalysis)

See Note 2 in radiation yield.

\section{area-related parameters}

areal parameters

Quantities expressed in terms of "per area" are denoted as area-related parameters.

Note 1: In photocatalysis, the area-related parameters do not characterise the catalyst as they do in heterogeneous dark catalysis. In general, the properties of a photocatalyst are not directly proportional to its area, especially in view of the fact that light may not equally reach every part of the surface of a particle. The light may not enter the pores of a porous surface and in this case only the outer area behaves as catalyst. A similar situation may occur when particles are aggregated.

Note 2: When the surface area that is accessed by the light cannot be established, it is recommended to use the total surface area in terms of the $\operatorname{BET}\left(\mathrm{N}_{2}\right)$ area. This implies that most probably a lower limit of the quantity is obtained, because the surface determined by $\mathrm{N}_{2}$ adsorption will be larger than that reached by the radiation.

See $\operatorname{BET}\left(\mathrm{N}_{2}\right)$ area.

\section{areal parameters}

area-related parameters

\section{artificial photosynthesis}

Photocatalytic production of substances from simple compounds (e.g., $\mathrm{H}_{2}$ and $\mathrm{O}_{2}$ from water, $\mathrm{H}_{2}$ from hydrogen sulfide, etc.) using ultraviolet, visible, or infrared radiation absorbed by chromophoric sys- 
tems, often included in microheterogeneous media, mimicking the action of antennae and reaction centres in natural photosynthetic organisms.

Note: Antennae (also called light-harvesting centres) are molecular structures enhancing the wavelength range and sensitivity of the photosynthetic units, due to an increase in light absorption.

See natural photosynthesis, photosynthesis.

See $[19,20]$.

attenuance (decadic $D_{10}$ or napierian $D_{\mathrm{e}}$ )

optical thickness $(\tau)$

Logarithm to the base 10 (or e) of the incident spectral radiant power, $P_{\lambda}^{0}$ (or incident spectral photon flux) divided by the transmitted spectral radiant power, $P_{\lambda}$ (or transmitted spectral photon flux) [7]

$$
D_{10}(\lambda)=\lg \left(\frac{P_{\lambda}^{0}}{P_{\lambda}}\right)=-\lg T(\lambda) \text { or } D_{\mathrm{e}}(\lambda)=\ln \left(\frac{P_{\lambda}^{0}}{P_{\lambda}}\right)=-\ln T(\lambda)
$$

where $T(\lambda)$ is the transmittance.

Note 1: Attenuance reduces to absorbance if the incident beam is only either transmitted or absorbed, but not reflected or scattered.

Note 2: In practice, for polychromatic irradiation, the attenuance is integrated over a wavelength range.

Note 3: Called extinction or extinctance or optical thickness when comprising absorption and scattering. The sum of the absorption and scattering coefficients at the same wavelength $\lambda_{\mathrm{i}}$ is called the extinction coefficient or attenuance coefficient, i.e.,

$$
\beta\left(\lambda_{\mathrm{i}}\right)=\alpha\left(\lambda_{\mathrm{i}}\right)+\sigma\left(\lambda_{\mathrm{I}}\right) .
$$

See $[7,8]$.

\section{attenuance coefficient}

extinction coefficient

See attenuance (Note 3).

\section{average volumetric rate of photon absorption}

absorbed (spectral) photon flux density

absorbed (spectral) photon radiance

See [16].

\section{band bending}

Bending of the (valence or conduction) bands in semiconductors at the surface owing to the existence of a surface charge potential, due to adsorption of (donor or acceptor) entities, or to a different distribution of defects in the subsurface region compared to the distribution in the bulk. Bending is also due to the potential drop when the semiconductor is brought in contact with an electrolyte (creation of a space charge region below the semiconductor surface) [21].

See [21-23]. 


\section{bandgap}

bandgap energy

Note: Band gap is an accepted writing form.

See extrinsic absorption, intrinsic absorption [21-23].

\section{bandgap energy, $E_{\mathrm{g}}$}

bandgap

Energy difference between the nonconductive and the conductive state of a material.

In semiconductors and insulators (dielectrics), it is the energy difference between the bottom of the conduction band and the top of the valence band.

Note: Bandgap energy is the preferred expression.

See [8].

\section{band-to-band recombination}

Recombination of charge carriers occurring via a one-step electron transition from the conduction band to the valence band in a semiconductor or insulator.

See $[22,23]$.

\section{BET surface area}

Abbreviation for Brunauer-Emmett-Teller surface area.

\section{$\operatorname{BET}\left(\mathbf{N}_{2}\right)$}

See Brunauer-Emmett-Teller surface area as determined by nitrogen absorption.

\section{black body}

Idealised object absorbing all incident radiant power, regardless of wavelength and transforming it all into heat. No electromagnetic radiation passes through it and none is reflected. A black body is a perfect absorber of radiant energy. Therefore, it is also a perfect emitter of radiation. The wavelength distribution of the radiation of a black body depends only on its temperature and not on its internal nature or structure.

\section{black-body-like photocatalytic reactor}

Reactor used to determine quantum yields of photoadsorption and photocatalysed reactions when the catalyst is in the form of a powdered layer (or powder dispersion). The incident photons are (nearly) all absorbed by the catalyst, provided that the dispersion layer is sufficiently thick (or the dispersion is sufficiently concentrated).

Note 1: To use the black-body-like photocatalytic reactor for the determination of quantum yields, the radiation arriving at the device should be monochromatic $(\lambda+\mathrm{d} \lambda)$ and the temperature well controlled.

See $[16,24]$.

\section{bleaching}

See [8]. 


\section{Brunauer-Emmett-Teller surface area}

Specific surface area $\left(\mathrm{m}^{2} \cdot \mathrm{kg}^{-1}\right)$ determined from the adsorption isotherm of a gas with the help of the Brunauer, Emmett, and Teller model using the conventional value for the molecular cross-section.

Note 1: The most common gas used is $\mathrm{N}_{2}$ with the conventional molecular cross-section of $0.162 \mathrm{~nm}^{2}$. With nitrogen, the total area is measured; this area includes the area in pores (that may not be reached by light). In this case, the expression $\operatorname{BET}\left(\mathrm{N}_{2}\right)$ is used.

Note 2: The area-related parameters do not characterise the catalyst in photocatalysis as they do in heterogeneous dark catalysis. The properties of a photocatalyst are not directly proportional to their area, in view of the fact that light does not equally reach every part of the surface area or particle.

See [25].

carrier

See charge carrier.

Note: Definition different from that given in [8], which refers to mass carriers.

\section{catalysis}

Action of a catalyst.

See $[9,11]$.

\section{catalyst}

Substance that participates in a particular chemical reaction and thereby increases its rate (due to a change in mechanism) but without a net change in the amount of that substance in the system.

Note: At a molecular level, the catalyst is used and regenerated during each set of microscopic chemical events leading from a molecular entity of reactant to a molecular entity of product.

Modified from [9,11].

\section{catalyst photoactivation (photomodification)}

Generation or alteration of the catalytic activity (selectivity) of a substance after its interaction with light.

Note: Typically, the term refers to catalytic activity (selectivity) occurring in the dark after irradiation has terminated.

See catalysis, photocatalysis.

\section{catalytic site}

Site at which catalysed chemical transformations take place.

Note 1: The area-related parameters do not characterise the catalyst in heterogeneous photocatalysis as they do in heterogeneous dark catalysis, inasmuch as the density and concentration of active sites are often unknown. Thus, the properties of a photocatalyst are not directly proportional to their area, in view of the fact that light does not equally reach every part of the surface or particle. Note also that photocatalysts may include several phases, in which case the overall area is physically not meaningful. 
Note 2: If the assumption can be made that the active sites are distributed over the entire (photo)catalyst surface, then the heterogeneous reactions are described "per area". The generally accepted $\operatorname{BET}\left(\mathrm{N}_{2}\right)$ surface area [26] is used. In spite of the fact that there is no direct correlation between the number of active sites and the BET $\left(\mathrm{N}_{2}\right)$ area, the use of the latter may help when no alternative exists.

Note 3: Quantities expressed in terms of "per BET $\left(\mathrm{N}_{2}\right)$ area" should be denoted as area-related, which also implies that a lower limit of the quantity is given, because the surface determined by $\mathrm{N}_{2}$ adsorption will be larger than that reached by the radiation.

See BET surface area.

\section{catalysed photochemical reaction}

Enhancement (alteration) of efficiency of photochemical reactions upon direct excitation of photoactive reactants via an intermediate interaction of these reactants with certain compounds that act as catalysts (promoters) of the appropriate chemical transformations of the reactants.

Note: $\quad$ Sometimes this process can be identical with photocatalysis.

\section{charge carrier}

Charge-carrying entity, especially an electron or a hole, in a semiconductor.

Note 1: In photocatalysis, the electrons or holes are produced on irradiating a semiconductor or insulator with appropriate energy through intrinsic absorption or extrinsic absorption of the actinic light.

Note 2: The majority carriers (higher density) are electrons in n-type semiconductors, whereas the minority carriers are holes. The majority charge carriers are holes in p-type semiconductors, whereas the minority carriers are electrons [21].

See $[22,23]$.

\section{chemisorption}

Adsorption of an entity (molecule, atom, or ion) onto a surface by way of a chemical interaction between a surface and the entity.

Note 1: Chemical interaction means that the Gibbs energy of interaction is relatively high, i.e., of the order of that of a chemical reaction.

Note 2: An example of chemisorption is the dissociative adsorption of water onto a metal-oxide particle surface.

Note 3: In some cases chemisorption may inactivate the photocatalytic (or photoadsorption) centre.

Note 4: The alternative form of adsorption is physisorption, where the Gibbs energy of interaction is relatively low.

See $[8,9]$. 


\section{Coekelbergs effect}

Adsorption at the surface of a pre-irradiated adsorbent (the photocatalyst) occurring in the dark, after the irradiation in the presence of the photoadsorbate entities is terminated, i.e., after preliminary photoadsorption.

See memory effect [27].

Compare with Solonitsyn effect.

\section{Coekelbergs-Solonitsyn effect}

See Coekelbergs effect, memory effect, Solonitsyn effect.

\section{colour centres}

Lattice defects (typically vacancies) that trap one or more electrons or holes in ionic crystals. They constitute a long-lived deep trap with a low efficiency of trapping of a carrier of the opposite sign.

Note 1: Colour centres accumulate in irradiated solids and give rise to photoinduced extrinsic absorption bands (photoinduced colour) in semiconductors and insulators. The dominant decay pathway of colour centres upon irradiation at moderate temperatures is photoionisation with the formation of free carriers and empty traps.

Note 2: The surface $\mathrm{F}$ (anion vacancies occupied by electrons) and $\mathrm{V}$ centres (trapped holes) in semiconductors and insulators are also colour centres with the distinction that they act as metastable active states of photocatalytic centres.

Note 3: Monitoring the photocolouration of a metal oxide during a surface photochemical reaction probes whether the reaction is photocatalytic [28].

See charge carriers, deep energy trap [23,29].

\section{compact layer \\ inner layer \\ Stern layer \\ Helmholtz layer}

\section{conduction band}

Vacant, or only partially occupied, set of many closely spaced electronic levels resulting from an array of a large number of atoms forming a solid system in which electrons can move freely or nearly so. Term used to describe the electrical properties (among several others) of metals, semiconductors, and insulators.

Note: In semiconductor and photocatalysis terminology, conduction band indicates the lowest conduction band, to which the electrons located in the highest valence band are transferred with energy higher than the bandgap energy [21].

See $[22,23]$.

\section{conversion spectrum}

Plot of a quantity related to the absorption (absorbance, cross-section [7], etc.) multiplied by the quantum yield for the considered process (e.g., the conversion cross-section [7]) against a suitable measure of photon energy, such as frequency, $v$, wavenumber, $\widetilde{v}$, or wavelength, $\lambda$.

See [8]. 


\section{coverage}

Occupied adsorption sites over the total number of adsorption sites. Related to the Langmuir-Hinshelwood kinetic model. Dimension one (it is a fractional number).

\section{deep energy trap}

Trap defined by an energy level (of trapped carriers) within the bandgap energy of a semiconductor or insulator with an energy difference between it and the conduction band (for electrons) or valence band (for holes) much greater than the energy of thermal excitation of the solid at a given temperature.

Note 1: The dominant decay pathway of the trap is either by trapping of carriers of the opposite sign (see recombination centre) or by photoionisation (see colour centres).

Note 2: Some active states of a photocatalytic centre are called a deep trap (as in solid-state physics).

See [23].

\section{depletion layer}

Subsurface space where the majority carriers are forced away owing to band bending caused by the surface potential. (i) The subsurface layer of an n-type semiconductor becomes depleted of electrons, which are the majority carriers, as a consequence of upward band bending. (ii) The subsurface layer of a p-type semiconductor becomes enriched by electrons (depleted of holes) as a consequence of downward band bending.

Note: The surface charge sign is identical to the sign of the majority charge carriers. The surface charge (surface potential) in semiconductors is produced as a result of adsorption or contact with the medium (an electrolyte or another surface).

See inversion layer [21].

\section{dielectrics}

insulator

Materials with electric conductivity, $\kappa$, far smaller than either a metal or a semiconductor.

See $[22,23]$.

\section{differential quantum yield}

See quantum yield.

\section{diffuse layer}

Layer in solution adjacent to a charged surface that contains an excess of counterions and a deficit of co-ions diffusely bound to the surface, as a result of coulombic interactions and thermal motion.

Note: The diffuse layer starts at a small distance from the surface, after the outer Helmholtz plane (OHP), several nm from the surface. Hydrated counter and co-ions in direct contact with the surface are located in the Stern layer.

See $[8,21,30]$. 


\section{direct bandgap semiconductor}

Semiconductor or dielectric materials whose first band-to-band transition occurs from the highest level in the valence band to the lowest level in the conduction band possessing the same electron momentum, i.e., $\boldsymbol{p}_{\max }=\boldsymbol{p}_{\min }$, with $\boldsymbol{p}_{\max }$ and $\boldsymbol{p}_{\min }$ the momentum vectors of electrons of the highest level in the valence band and the lowest level in the conduction band, respectively.

Note: On photoexcitation, the electrons change their energy owing to the absorption of photons and maintain the same momentum.

Compare with indirect bandgap semiconductor [22,23].

doping (a semiconductor)

Process to increase the electric conductivity of a semiconductor by the intentional addition of certain impurities in small quantities $\left(\mu \mathrm{mol} \cdot \mathrm{mol}^{-1}\right)$.

Note 1: In photocatalysis doping is a word loosely applied to describe the modification of the photocatalyst by addition of the desired chemical dopant (impurity) either in the bulk or on the surface. However, using this term for any kind of deposit at the surface is not appropriate.

Note 2: For doping silicon, the dopants are called 13-15 compounds, referring to the columns in the Periodic Table of the Elements [31].

See [8].

dose (energy or photon dose)

Energy or amount of photons absorbed per surface area or per volume by an irradiated object during a particular exposure time.

Note 1: SI unit is $\mathrm{J} \cdot \mathrm{m}^{-2}$ or $\mathrm{J} \cdot \mathrm{m}^{-3}$ and $\mathrm{mol} \cdot \mathrm{m}^{-2}$ or $\mathrm{mol} \cdot \mathrm{m}^{-3}$, respectively. Common unit in case of photon number basis is $\mathrm{m}^{-2}$ or $\mathrm{m}^{-3}$, respectively, and in chemical amount basis einstein $\cdot \mathrm{m}^{-2}$ or einstein $\cdot \mathrm{m}^{-3}$, respectively.

Note 2: In some research areas (e.g., photopolymerisation and water purification through irradiation), dose is used in the sense of fluence, i.e., the energy or amount of photons per surface area (or per volume) received by an irradiated object during a particular exposure time. SI unit is $\mathrm{J} \cdot \mathrm{m}^{-2}$ or $\mathrm{J} \cdot \mathrm{m}^{-3}$ and $\mathrm{mol} \cdot \mathrm{m}^{-2}$ or $\mathrm{mol} \cdot \mathrm{m}^{-3}$, respectively. Common unit in case of photon number basis is $\mathrm{m}^{-2}$ or $\mathrm{m}^{-3}$, respectively, and in chemical amount basis einstein $\cdot \mathrm{m}^{-2}$ or einstein $\cdot \mathrm{m}^{-3}$, respectively.

See also $U V$ dose [7].

\section{downward band bending}

See depletion layer.

\section{driving force}

The negative of the standard Gibbs energy change for a particular reaction $\left(\Delta_{\mathrm{r}} G^{\circ}\right)$, e.g., for energy transfer or electron transfer $\left(\Delta_{\mathrm{ET}} G^{\circ}\right)$.

See $[7,9]$. 


\section{dynamic photoadsorption process}

Simultaneous (or synchronous) formation of adsorbed species (adsorbate) via photoadsorption and detachment by photodesorption to yield the same species, both processes occurring under photoexcitation of the solid photocatalyst (adsorbent).

\section{effective radiation-catalytic activity}

Number of product entities (atoms, molecules, or radicals) generated by each $100 \mathrm{eV}$ of radiation incident on the system. Unit $(100 \mathrm{eV})^{-1}$.

Note 1: In SI units, it refers to the amount of substance in $\mu \mathrm{mol}$ generated for each joule of radiation incident on the system. SI unit is $\mu \mathrm{mol} \cdot \mathrm{J}^{-1}$.

Note 2: It is convenient to use the amount of substance B in $10^{-7} \mathrm{~mol}$ of $\mathrm{B}$, generated for each joule of radiation incident on the system. By choosing this (submultiple of) SI unit, a number almost identical to the $G$ value (within $4 \%)$ in the obsolete unit $(100 \mathrm{eV})^{-1}$ is obtained, as $(100 \mathrm{eV})^{-1}=1.036 \times 10^{-7} \mathrm{~mol} \cdot \mathrm{J}^{-1}$.

See radiation-chemical yield.

efficiency (of a step; $\eta$ )

Ratio between the useful energy delivered or bound and the energy supplied, i.e., energy output/energy input.

Note 1: Concept also used in the sense of a quantitative measure of the relative rate of a given step involving a species with respect to the sum of the rates of all of the parallel steps that depopulate that species, i.e., $\eta=k_{\mathrm{i}} /\left(\Sigma_{\mathrm{i}} k_{\mathrm{i}}\right)$ with $k_{\mathrm{i}}$ the rate constants of each of the decay steps of the species.

Note 2: In the context of photocatalysis in heterogeneous media, quantum efficiency and photonic efficiency refer to the use of wavelength in a range $\left(\lambda_{1}-\lambda_{2}\right)$ (in contrast to the use of monochromatic radiation in conjunction with the word yield), for absorbed radiation in the case of quantum efficiency and for incident radiation in the case of photonic efficiency.

See also quantum yield $[7,8]$.

\section{efficiency spectrum}

See action spectrum, photonic yield spectrum.

See [7].

\section{einstein}

One mol of photons. Widely used, although it is neither an SI unit nor recognised to be used with the SI.

Note 1: Einstein sometimes is defined as the energy of one mole of photons. This particular use is discouraged.

Note 2: The energy of one einstein of frequency $v$ is $E=N_{\mathrm{A}} h v$ with $h$ the Planck constant and $N_{\mathrm{A}}$ the Avogadro constant.

See [7]. 


\section{electrical double layer}

Array of charged species and oriented dipoles existing at a solid-solution interface. The solid may be a metal or a semiconductor. Double refers to the fact that the surface charge and its counter charge at the solution side are included.

Note: $\quad$ Often the solution side is considered to be composed of a thin inner layer of (hydrated) ions in contact with the surface and a diffuse layer further out in solution.

See diffuse layer, inner layer [21,22,30].

See also interfacial double layer in [8].

\section{electron transfer}

Transfer of an electron from one molecular entity to another or between two localised sites in the same molecular entity.

See [8].

\section{electron transfer photosensitisation}

Photochemical process in which a reaction of a nonabsorbing substrate is induced by electron transfer to or from an ultraviolet, visible, or infrared radiation-absorbing sensitiser.

Note: Should the sensitiser be recycled in the overall process, then the photosensitiser is acting as a photocatalyst. Depending on the action of the sensitiser as electron donor or acceptor the sensitisation is called reductive or oxidative.

Related term: photosensitisation.

See [8].

electronic energy migration (or hopping)

Movement of electronic excitation energy from one molecular entity to another entity of the same species, or from one part of a molecular entity to another of the same kind (e.g., excitation migration between chromophores of an aromatic polymer). The migration may occur by radiative or nonradiative (radiationless) processes.

Note: This effect describes migration of excitons from one to another trapped position. See [8].

\section{Eley-Rideal (ER) mechanism}

Mechanism of photocatalysis involving the migration of entities in the liquid or gas phase to the surface-active centres.

Note: In photocatalysis, taking into account the very short lifetimes of the active (photoexcited) catalytic sites, the kinetic equation for the Eley-Rideal mechanistic model becomes the same as the kinetic equation for the Langmuir-Hinshelwood mechanism.

See, e.g., [32]. 


\section{energy transfer}

excitation transfer

Process by which a molecular entity is excited (e.g., by absorption of ultraviolet, visible, or infrared radiation or by chemiexcitation) and a phenomenon (a physical or a chemical process) originates from the excited state of another molecular entity, which has interacted with the originally absorbing entity.

Note 1: In mechanistic photochemistry, the term has been reserved for the photophysical process in which an excited state of one molecular entity (the donor) is deactivated to a lower-lying state by transferring energy to a second molecular entity (the acceptor), which is thereby raised to a higher energy state. The excitation may be electronic, vibrational, rotational, or translational. The donor and acceptor may be two parts of the same molecular entity, in which case the process is an intramolecular energy transfer.

Note 2: Energy transfer is also called exciton migration in solids, when no charge transfer occurs.

For related terms, see [7].

\section{ER mechanism}

Eley-Rideal mechanism

\section{excitation transfer}

energy transfer

\section{excited state}

State of higher energy than the ground state of a chemical entity.

Note 1: In photochemistry, an electronically excited state is usually meant [7].

Note 2: Photogenerated free electrons in the conduction band and free photogenerated holes in the valence band are excited states of the photocatalyst. Free and bound excitons are also excited states of photocatalysts. The photocatalyst with a photocarrier trapped by bulk and surface defects, even in the absence of free photocarriers at the end of irradiation, is in an excited state in relation to the ground state of the solid without trapped photocarrier. Thus, the active state of a photocatalytic (photoadsorption) centre is an excited state of the photocatalyst. At the same time, this state is in its lowest energy state with respect to the set of possible electronic states in the solid subsystem (e.g., empty defect and carrier).

See [8].

\section{exciton}

In some applications, it is useful to consider electronic excitation as a quasi-particle that is capable of migrating.

Note 1: In organic materials two models are used: the band or wave model (low temperature, high crystalline order) and the hopping model (higher temperature, low crystalline order or amorphous state). Energy transfer in the hopping limit is identical with energy migration. In semiconductors and insulators, a free exciton is a bound electron-hole (neutral quasi-particle) capable of migrating and transferring its energy to the solid lattice. 
Note 2: A localised exciton is an exciton trapped by a defect or self-trapped at a regular lattice site by lattice polarisation, which leads to the electronically excited state of the defect or to the localised excitation of the regular lattice, respectively. In the latter case, exciton decay may result in the formation of new defects. The decay of self-trapped excitons on the surface may lead to the formation of surface-active centres with catalytic activity.

Note 3: Excitons are the emissive entities produced by recombination of bipolarons in electroluminescent materials.

See electronic energy migration [7].

\section{exciton absorption}

Absorption of ultraviolet, visible, or infrared radiation yielding optical information on the excitons in solids. Exciton absorption bands in a spectrum of a semiconductor or insulator are located on the long wavelength side of the fundamental absorption edge.

See, e.g., [33].

\section{extinctance}

attenuance

\section{extinction coefficient, $\beta(\lambda)$}

attenuance coefficient

Sum of the absorption and scattering coefficients at the same wavelength, i.e., $\lambda_{\mathrm{i}}$ :

$$
\beta\left(\lambda_{\mathrm{i}}\right)=\alpha\left(\lambda_{\mathrm{i}}\right)+\sigma\left(\lambda_{\mathrm{i}}\right)
$$

with $\alpha\left(\lambda_{\mathrm{i}}\right)$ the linear napierian absorption coefficient and $\sigma\left(\lambda_{\mathrm{i}}\right)$ the linear napierian scattering coefficient. SI unit is $\mathrm{m}^{-1}$.

Note 1: This term should be reserved for scattering media and not used for transparent media in which no scattering or dispersion occurs.

Note 2: It can also be defined in terms of the decadic absorption and scattering coefficients.

See attenuance .

extrinsic absorption (of solid photocatalysts)

Absorption of ultraviolet, visible, or infrared radiation in semiconductors and insulators corresponding to optical transitions of electrons from defect energy levels (within the bandgap energy) to conduction band levels, or holes to valence band levels (photoionisation of defects), or from the ground state level to excited levels of a given intrinsic defect of any sort.

Note 1: Typically, extrinsic absorption bands are a few tenths to a few $\mathrm{eV}$ distant from the fundamental absorption edge in semiconductors and insulators. The red limits of photocatalytic reactions are determined by photoexcitation of photocatalysts in the extrinsic absorption bands.

Note 2: Doping of a catalyst creates an extrinsic defect.

See, e.g., [34]. 


\section{extrinsic (surface) defect}

Imperfection in a solid semiconductor (or insulator) connected with surface defects of any sort involving extrinsic chemical units in relation to the chemical formula of the solid, particularly adsorbed species of any sort.

Note 1: These extrinsic surface defects play an essential role in heterogeneous photocatalysis. For example, they can serve as photocatalytic centres (sites). Surface defects, as well as bulk defects located in the near-surface region, can serve as entities that absorb ultraviolet, visible, or infrared radiation and generate free charge carriers. Surface defects can trap charge carriers and serve as recombination centres, thereby influencing the concentration of photocarriers at the surface of photocatalyst particles.

Note 2: Doping of a catalyst creates an extrinsic defect.

See [34].

\section{F centres}

Anion vacancies occupied by electrons in semiconductors.

See colour centres [35].

\section{Fermi energy}

Energy associated with the Fermi level.

Fermi level, $E_{\mathrm{F}}$

Level associated with the energy of the least tightly held electrons within a solid. The value of the Fermi level at absolute zero temperature $(0 \mathrm{~K})$ is the Fermi energy and is a constant for each solid.

Note 1: The Fermi level changes as the solid is warmed and as electrons are added to or withdrawn from the solid. The Fermi level (the chemical potential of the electrons) has the probability to be exactly half-filled with electrons, i.e., the Fermi-Dirac distribution function is 0.5 .

Note 2: Doping shifts the Fermi level and, therefore, its energy $E_{\mathrm{F}}$.

See $[8,21]$.

\section{Fermi level splitting, $\Delta \mathrm{F}$}

Splitting of the Fermi level in irradiated semiconductors and insulators into two Fermi quasi-levels for electrons $\left(E_{\mathrm{F}}^{\mathrm{e}}\right)$ and for holes $\left(E_{\mathrm{F}}^{\mathrm{h}}\right)$, displaced toward the bottom of the conduction band and toward the top of the valence band, respectively.

Note 1: The larger the radiant power of the radiation, the larger is the Fermi level energy splitting, $\Delta \mathrm{F}=E_{\mathrm{F}}{ }^{\mathrm{e}}-E_{\mathrm{F}} \mathrm{h}$.

Note 2: The positions of the Fermi quasi-levels within the bandgap energy determine the concentration of free thermalised photocarriers in irradiated solid photocatalysts.

See bandgap energy, conduction band, valence band [22].

\section{flash photolysis}

Transient-spectroscopy and transient kinetic technique in which an ultraviolet, visible, or infrared radiation pulse is used to produce transient species. 
Note 1: Commonly, an intense pulse of short duration is used to produce a sufficient concentration of transient species suitable for spectroscopic observation. The most common use is for the observation of absorption of transient species (transient absorption spectroscopy).

Note 2: If only photophysical processes are involved, a more appropriate term would be "pulsed photoactivation". The term flash photolysis would only be correct, should chemical bonds be broken (the Greek "lysis" means dissolution or decomposition and in general "lysis" is used to indicate breaking). However, historically, the name has been used to describe the technique of pulsed excitation, independently of the process that follows the excitation.

See [36] for a list of recommended procedures and optical data on transient species. See [7].

\section{flatband potential}

If effects of surface states are negligible, $\Delta \varphi^{\mathrm{sc}}$, the potential drop associated with the space charge in the semiconductor, vanishes as the charge on the semiconductor becomes zero. The potential of the semiconductor corresponding to this condition is the flat band potential, which plays the same role as the potential of zero charge for metals [8].

Note 1: Potential at which no excess charge exists in the semiconductor (potential of zero charge) and there is no electric field and no space charge region so that the (conduction and valence) bands are not bent.

Note 2: Flat band potential is an accepted writing form.

See also band bending [21,23].

fluence, $H_{\mathrm{o}}, F_{\mathrm{o}}$ radiant energy fluence

Radiant energy, $Q$, incident on a small sphere from all directions divided by the cross-sectional area of that sphere. SI unit is $\mathrm{J} \cdot \mathrm{m}^{-2}$.

Note 1: The term is used in photochemistry to specify the energy delivered in a given time interval (e.g., by a laser pulse).

Note 2: Mathematical definition: $H_{\mathrm{o}}=\mathrm{d} Q / \mathrm{d} S$, where $S$ is the area of cross-section. If the radiant energy is constant over the area $S$, then $H_{\mathrm{o}}=Q / S$.

Note 3: Equivalent definition: $H_{\mathrm{o}}=\int_{t} E_{\mathrm{o}} \mathrm{d} t$ where $E_{\mathrm{o}}$ is the fluence rate and $t$ the duration of the irradiation. If the fluence rate is constant over the time interval, then $H_{\mathrm{o}}=E_{\mathrm{o}} t$.

Note 4: Fluence is identical to spherical radiant exposure and reduces to radiant exposure, $H$, for a beam not scattered or reflected by the target or its surroundings.

Note 5: The subindex o (letter o) indicates radiation from all directions.

Related terms: dose, photon fluence [8].

fluence rate, $E_{\mathrm{o}}$ radiant energy fluence rate

Total radiant power, $P$, incident from all directions onto a small sphere divided by the cross-sectional area of that sphere. SI unit is $\mathrm{W} \cdot \mathrm{m}^{-2}$. 
Note 1: Mathematical definition: $E_{\mathrm{o}}=\mathrm{d} P / \mathrm{d} S=\mathrm{d} H_{\mathrm{o}} / \mathrm{d} t$. If the radiant power is constant over the area $S$, then $E_{\mathrm{o}}=P / S$.

Note 2: For a divergent beam $E_{\mathrm{o}}=\int_{\Omega} L_{\Omega} \mathrm{d} \Omega$, with $L_{\Omega}$ the spherical radiance distributed over the solid angle $\Omega$. The maximum integration value is $\Omega=4 \pi$.

Note 3: Fluence rate is identical to spherical irradiance and reduces to irradiance, E, for a parallel and perpendicularly incident beam not scattered or reflected by the target or its surroundings.

Note 4: For a collimated or quasi-collimated beam, the fluence rate and irradiance become the same in the limit of purely parallel beams.

Note 5: The subindex o (letter o) indicates radiation from all directions.

Related term: photon fluence rate [8].

See intensity, irradiance, radiance.

\section{flux density}

photon radiance

\section{formal quantum efficiency (FQE)}

See photonic efficiency.

\section{FQE}

Abbreviation of formal quantum efficiency.

See photonic efficiency.

\section{free charge carriers}

Applies to free electrons or free holes.

\section{free electrons}

Photoexcited electrons from the valence band into the conduction band in semiconductors and insulators.

Note 1: Thermoexcitation or doping with certain impurities also leads to formation of free electrons in the conduction band of semiconductors. In metals and n-type semiconductors free electrons exist originally.

Note 2: The expression "free electron carrier" is redundant and not recommended.

See [23].

fundamental absorption (of solid photocatalysts)

intrinsic absorption

Absorption of ultraviolet, visible, or infrared radiation in semiconductors and insulators inducing optical transitions of electrons originating exclusively from the valence band to the conduction band to yield free electron-hole pairs and/or exciton absorption bands.

See extrinsic absorption [34]. 
fundamental absorption edge (threshold - of solid photocatalysts)

High wavelength limit of the fundamental absorption band in the spectrum of semiconductors and of insulators.

See [34].

\section{Gibbs energy of photoinduced electron transfer}

For photoinduced electron transfer between an acceptor (A) and a donor (D) (either one of them may be the electronically excited molecular entity) of any charge type, $z(\mathrm{~A})$ and $z(\mathrm{D})$, the change in standard Gibbs energy can be approximated as (the notation is for the case of neutral species D and A)

$$
\Delta_{\mathrm{ET}} G^{\circ}=N_{\mathrm{A}}\left\{e\left[E^{\circ}\left(\mathrm{D}^{\bullet+} / \mathrm{D}\right)-E^{\circ}\left(\mathrm{A} / \mathrm{A}^{\bullet-}\right)\right]+w\left(\mathrm{D}^{\bullet+} \mathrm{A}^{\bullet-}\right)-w(\mathrm{DA})\right\}-\Delta E_{0,0}
$$

where $e=1.602176487 \times 10^{-19} \mathrm{C}$ is the elementary charge, $N_{\mathrm{A}}=6.02214179 \times 10^{23} \mathrm{~mol}^{-1}$ is the Avogadro constant, $E^{\circ}\left(\mathrm{D}^{\circ+} / \mathrm{D}\right)$ in $\mathrm{V}$ is the standard electrode potential of the donor cation radical resulting from the electron transfer, $E^{\circ}\left(\mathrm{A} / \mathrm{A}^{*-}\right)$ in $\mathrm{V}$ is the standard electrode potential of the acceptor (both relative to the same reference electrode) and $\Delta E_{0,0}$ in $\mathrm{J} \cdot \mathrm{mol}^{-1}$ is the vibrational zero-zero electronic energy of the excited partner (provided that a vibrationally equilibrated excited state at energy $E_{0,0}$ takes part in the reaction), all data referring to the same solvent.

$w\left(\mathrm{D}^{\bullet+} \mathrm{A}^{\bullet-}\right)$ and $w(\mathrm{DA})$ are the electrostatic work terms that account for the effect of coulombic attraction in the products and reactants, respectively,

$$
\begin{aligned}
& w\left(\mathrm{D}^{\bullet+} \mathrm{A}^{\bullet-}\right) / \mathrm{J}=z\left(\mathrm{D}^{\bullet+}\right) z\left(\mathrm{~A}^{\bullet-}\right) e^{2} /\left(4 \pi \varepsilon_{0} \varepsilon_{\mathrm{r}} a\right) \\
& w(\mathrm{DA}) / \mathrm{J}=z(\mathrm{D}) z(\mathrm{~A}) e^{2} /\left(4 \pi \varepsilon_{0} \varepsilon_{\mathrm{r}} a\right)
\end{aligned}
$$

where $a$ is the distance of the charged species after electron transfer, $\varepsilon_{\mathrm{r}}$ is the relative medium static permittivity (formerly called dielectric constant), $\varepsilon_{0} \approx 8.854 \times 10^{-12} \mathrm{C}^{2} \mathrm{~J}^{-1} \cdot \mathrm{m}^{-1}$ is the vacuum permittivity (formerly called electric constant), and $z(\mathrm{X})$ the charge of the species $\mathrm{X}$.

In SI units, the factor $e^{2} /\left(4 \pi \varepsilon_{0}\right) \approx 2.307 \times 10^{-28} \mathrm{~J} \mathrm{~m}$. For the case of neutral species $\mathrm{A}$ and $\mathrm{D}$, $z(\mathrm{~A})=z(\mathrm{D})=0$.

Note 1: Several approximations are in use for the calculation of the term $w\left(\mathrm{D}^{\bullet+} \mathrm{A}^{\bullet-}\right)$, depending on the nature of the species formed such as contact or solvent-separated radical ion pairs or extended and/or linked D and A molecular entities. In the latter case, the stabilisation of a dipole $\mu$ in a cavity of radius $\rho$ could be an appropriate model [37] and

$$
w\left(\mathrm{D}^{\bullet+} \mathrm{A}^{\bullet-}\right)=\left[N_{\mathrm{A}}\left(\mu^{2}\right) /\left(4 \pi \varepsilon_{0} \rho^{3}\right)\right]\left[\left(\varepsilon_{\mathrm{r}}-1\right) /\left(2 \varepsilon_{\mathrm{r}}+1\right)\right]
$$

Note 2: In the above definitions, the IUPAC recommendations for the sign and symbols of standard potentials are used. Although not complying with the IUPAC recommended nomenclature for the standard electrode potentials, traditionally the equation has been written as

$$
\Delta_{\mathrm{ET}} G^{\circ}=N_{\mathrm{A}}\left\{e\left(E^{\circ}{ }_{\text {ox }}-E_{\text {red }}^{\circ}\right)+\left[z\left(\mathrm{D}^{\bullet+}\right) z\left(\mathrm{~A}^{\bullet-}\right)-z(\mathrm{D}) z(\mathrm{~A})\right] e^{2} /\left(4 \pi \varepsilon_{0} \varepsilon_{\mathrm{r}} a\right)\right\}-\Delta E_{0,0}
$$

with $E^{\circ}{ }_{\text {ox }}$ the standard electrode potential at which the oxidation occurs, and $E_{\text {red }}^{\circ}$ the standard electrode potential at which the reduction occurs. This form of the first term within the wiggly brackets is misleading and not recommended (corrected from [7]).

Note 3: The standard potentials of oxidation and reduction are often called, respectively, "oxidation" and "reduction" potential. These terms are intrinsically confusing and should be avoided altogether, because they conflate the chemical concept of reaction with the physical concept of electrical potential. 
Note 4: The equation used for the calculation of the Gibbs energy of photoinduced electrontransfer processes should not be called the Rehm-Weller equation [7].

Related terms: driving force [8], polar driving force in [7].

For a list of electrode potentials, see [38].

See [39].

\section{ground state}

State of a chemical entity associated with the lowest energy. Corrected from [8].

Note: In photochemistry, ground electronic state is usually meant.

\section{$G$-value}

radiation-chemical yield

\section{Helmholtz layer}

inner layer

Stern layer

compact layer

\section{heterogeneous photocatalysis}

Photocatalysis taking place at the interfacial boundary between two phases (solid/liquid, solid/gas, liquid/gas).

\section{heterogeneous radiation catalysis}

Radiation catalysis taking place in heterogeneous systems.

\section{hole transfer}

Charge migration process in which the majority carriers are positively charged.

See [8].

\section{homogeneous photocatalysis}

Photocatalysis taking place in homogeneous systems.

\section{homogeneous radiation catalysis}

Radiation catalysis taking place in homogeneous systems.

\section{IHP}

Abbreviation of inner Helmholtz plane.

\section{indirect bandgap semiconductor}

Semiconductor or dielectric materials with a first band-to-band transition from the highest level in the valence band to the lowest level in the conduction band requiring the change of electron momentum, that is $\boldsymbol{p}_{\text {max }} \neq \boldsymbol{p}_{\text {min }}$, where $\boldsymbol{p}_{\text {max }}$ and $\boldsymbol{p}_{\text {min }}$ are the momentum vectors of electrons of the highest level in the valence band and the lowest level in the conduction band, respectively. 
Note: Direct band-to-band electronic transitions between two bands of different momentum (e.g., $\boldsymbol{p}_{\max } \neq \boldsymbol{p}_{\min }$ ) are forbidden because momentum must be conserved. Upon excitation, such changes occur owing to interaction of the electronic subsystem with the phonons.

Compare with direct bandgap semiconductor [23].

\section{inner Helmholtz plane (IHP)}

Locus of electrical centres of specifically adsorbed ions and outer boundary of the inner Helmholtz layer.

See outer Helmholtz plane [8,30].

\section{inner layer}

compact layer

Stern layer

Helmholtz layer

Closest layer to the semiconductor surface (in an electrical double layer) that contains solvent molecules and other species (ions or molecules) specifically adsorbed.

Note 1: This layer corresponds with the inner + outer Helmholtz planes.

Note 2: Solvent properties in the Stern layer can be different from the bulk solvent. In the diffuse layer, located after the inner layer, solvent properties are considered to be the same as in the bulk.

See specific adsorption $[8,30]$.

\section{in-scattering}

scattering in (into the beam under consideration)

Physical phenomenon considering scattered radiative energy from rays in any direction $\Omega^{\prime}$ into the direction $\Omega$ of the rays considered in the radiative transfer equation (RTE).

Note 1: Mathematical definition:

$$
\mathrm{Sc}_{\text {in }}=\frac{\sigma_{\lambda}(x, t)}{4 \pi} \int_{4 \pi} B_{\lambda}\left(\Omega^{\prime} \rightarrow \Omega\right) L_{\Omega, \lambda}(x, t) \mathrm{d} \Omega^{\prime}
$$

where $\boldsymbol{x}$ is the spatial coordinate in a three-dimensional space along the propagation of the beam, $t$ is the time coordinate, $\sigma_{\lambda}(x, t)$ is the space- and time-dependent napierian linear scattering coefficient, $L_{\Omega, \lambda}(\boldsymbol{x}, t)$ is the incident time- and space-dependent radiance and $B_{\lambda}$ is the phase function, i.e., a distribution function acting as a source of photons from any direction $\Omega^{\prime}$ into the direction $\Omega$ under consideration. Unit is $\mathrm{W} \cdot \mathrm{sr}^{-1} \cdot \mathrm{m}^{-4}$. Common unit is $\mathrm{W} \cdot \mathrm{sr}^{-1} \cdot \mathrm{cm}^{-3} \cdot \mathrm{nm}^{-1}$.

Note 2: Mathematical expression in photon terms:

$$
\mathrm{Sc}_{\mathrm{in}}=\frac{\sigma_{\lambda}(\boldsymbol{x}, t)}{4 \pi} \int_{4 \pi} B_{\lambda}\left(\Omega^{\prime} \rightarrow \Omega\right) L_{\mathrm{p}, \Omega, \lambda}(x, t) \mathrm{d} \Omega^{\prime}
$$

with $L_{\mathrm{p}, \Omega, \lambda}(\boldsymbol{x}, t)$ the incident spectral photon radiance. 
SI unit is $\mathrm{s}^{-1} \cdot \mathrm{sr}^{-1} \cdot \mathrm{m}^{-4}$ in photon number basis and $\mathrm{mol} \cdot \mathrm{s}^{-1} \cdot \mathrm{sr}^{-1} \cdot \mathrm{m}^{-4}$ in chemical amount basis. Common unit is $\mathrm{s}^{-1} \cdot \mathrm{sr}^{-1} \cdot \mathrm{cm}^{-3} \cdot \mathrm{nm}^{-1}$ in photon number basis and einstein $\cdot \mathrm{s}^{-1} \cdot \mathrm{sr}^{-1} \cdot \mathrm{cm}^{-3} \cdot \mathrm{nm}^{-1}$ in chemical amount basis.

See out-scattering, radiative transfer equation [16,40,41].

\section{insulator \\ dielectrics}

\section{integral quantum yield}

See quantum yield.

\section{integrating sphere}

Hollow sphere having a highly reflecting inside surface used as a device to collect, with very high efficiency, ultraviolet, visible, or infrared radiation scattered or emitted from a sample contained in it or located outside and near one of the ports. Small ports allow the entrance of the radiation and access to a detector.

See [7].

\section{intensity}

Traditional term indiscriminately used for photon flux, fluence rate, irradiance, or radiant power. In terms of an object exposed to radiation, the term should be used only for qualitative descriptions.

intensity (of an ultraviolet, visible, or infrared radiation source), $I$ radiant intensity

intensity (of a spectral feature)

Describes the magnitude of the particular feature in the spectrum.

\section{interparticle electron transfer (IPET)}

Electron transfer taking place from a photoexcited semiconductor onto another semiconductor (or insulator) in its ground state leading to vectorial (irreversible) charge transfer.

\section{intrinsic absorption}

fundamental absorption

\section{intrinsic emission}

Band-to-band recombination of electrons and holes (or exciton decay) in a semiconductor or insulator leading to luminescence.

\section{intrinsic (surface) defects}

Imperfections connected with surface point defects (zero-dimensional), linear defects (one-dimensional), or square defects (two-dimensional) not including extrinsic chemical units in relation to the chemical formula of the solid. 
Note: Surface vacancies, anionic vacancies (in n-type semiconductors), cationic vacancies (in p-type semiconductors), interstitial cations, edges, corners and kinks are some examples of intrinsic surface defects.

Related term: extrinsic (surface) defect.

See [34].

\section{inversion layer}

Layer formed when band bending places the Fermi level on the surface close to the band of the bulk minority charge carriers. This leads to a higher concentration of the minority charge carriers on the surface relative to the concentration of the majority charge carriers, an opposite situation to that in the bulk. See accumulation layer, depletion layer [21].

\section{IPET}

Acronym for interparticle electron transfer.

Note: $\quad$ Also used for intramolecular photoinduced electron transfer.

irradiance (at a point of a surface), $E$

Radiant power, $P$, of all wavelengths incident from all upward directions on a small element of surface containing the point under consideration divided by the area of the element. SI unit is $\mathrm{W} \cdot \mathrm{m}^{-2}$.

Note 1: Integral, taken over the hemisphere visible from the given point, of the expression $L_{\mathrm{e}} \cos \theta \mathrm{d} \Omega$, where $L_{\mathrm{e}}$ is the radiance at the given point in the various directions of the incident elementary beams of solid angle $\Omega$ and $\theta$ is the angle between any of the beams and the normal to the surface at the given point, i.e., $E=\int_{2 \pi} L_{\mathrm{e}} \cos \theta \mathrm{d} \Omega$.

Note 2: Alternative mathematical definition: $E=\mathrm{d} P / \mathrm{d} S$. If $P$ is constant over the surface area considered, then $E=P / S$.

Note 3: This term refers to a beam not scattered or reflected by the target or its surroundings. For a beam incident from all directions, fluence rate $\left(E_{\mathrm{o}}\right)$ is the preferred term, with the subindex o indicating radiation from all directions.

Note 4: For polychromatic irradiation, $\int_{\lambda} E_{\lambda} \mathrm{d} \lambda$, where $E_{\lambda}$ is the spectral irradiance at wavelength $\lambda$.

Note 5: For a collimated or quasi-collimated beam, the irradiance and fluence rate become the same in the limit of purely parallel beams.

For related terms, see [8].

\section{Langmuir-Hinshelwood (LH) kinetic model}

Model describing the dependence of the rate of product formation, $r_{\mathrm{LH}}$, on the reagent amount concentration $c$ in bulk solution (pressure, $p$, for gases). $r_{\mathrm{LH}}$ is given by the equation

$$
r_{\mathrm{LH}}=-\mathrm{d} c / \mathrm{d} t=k K c /(1+K c)
$$

where $k$ is the apparent rate constant (SI unit is $\mathrm{L} \cdot \mathrm{mol}^{-1} \cdot \mathrm{s}^{-1}$ in amount concentration) of the process occurring at the active site on the photocatalyst surface and $K=k_{\mathrm{ads}} / k_{\mathrm{des}}$ is the adsorption/desorption equilibrium constant, both obtained at the same radiant power as that of the actinic light. 
Note 1: This model assumes a fast adsorption/desorption equilibrium step according to the Langmuir isotherm and a subsequent slow surface step. Thus, the pre-adsorbed entities react with surface-active centres, other than by the Eley-Rideal mechanism, in which it is assumed that the molecular species in the liquid or gas phase react with the surfaceactive centres.

Note 2: Observation of Langmuir-Hinshelwood kinetics does not mean that the Langmuir-Hinshelwood mechanism is operative. For example, the alternative Eley-Rideal mechanism yields the same type of kinetics provided that the active state of the surface catalytic (photoadsorption) centre has a limited lifetime.

Note 3: In photocatalysis, taking into account the very short lifetimes of the active (photoexcited) catalytic sites, the kinetic equation for the Eley-Rideal mechanistic model becomes the same as the kinetic equation for the Langmuir-Hinshelwood model.

See physisorption $[32,42,43]$.

\section{LH kinetic model}

Langmuir-Hinshelwood kinetic model

\section{lifetime, $\tau$}

Lifetime of a molecular entity, which decays by first-order kinetics, is the time needed for a concentration of the entity to decrease to $1 / \mathrm{e}$ of its original value, i.e., $c(t=\tau)=c(t=0) / \mathrm{e}$. Statistically, it represents the life expectation of the entity. It is equal to the reciprocal of the sum of the first-order rate constants of all processes causing the decay of the molecular entity.

Note 1: Mathematical definition: $\tau=1 / k=1 /\left(\Sigma_{i} k_{i}\right)$ with $k_{i}$ the first-order rate constants for all decay processes of the decaying state.

Note 2: Lifetime is used sometimes for processes that are not first order. However, in such cases, the lifetime depends on the initial concentration of the entity, or of a quencher and therefore only an initial or a mean lifetime can be defined. In this case it should be called apparent lifetime or decay time.

Note 3: Occasionally, the term half-life $\left(\tau_{1 / 2}\right.$ or $\left.t_{1 / 2}\right)$ is used, representing the time needed for the concentration of an entity to decrease to one half of its original value, i.e.,

$$
c\left(t=\tau_{1 / 2}\right)=c(t=0) / 2
$$

See [8].

\section{light-initiated thermocatalytic phenomena}

See thermophotocatalysis.

\section{local differential absorption}

Absorption term in the radiative transfer equation (RTE).

Mathematical expression is

$$
\alpha_{\lambda}(x, t) L_{\Omega, \lambda}(x, t)
$$

with $\alpha_{\lambda}(\boldsymbol{x}, t)$ the space- and time-dependent linear napierian absorption coefficient and $L_{\Omega, \lambda}(\boldsymbol{x}, t)$ the spectral radiance arriving at position $\boldsymbol{x}$ and time $t$ from every possible angular directions $\Omega$. See [16]. 
local volumetric rate of energy absorption (LVREA), $L_{\lambda}^{\mathrm{a}}(\boldsymbol{x}, t)$

absorbed local spectral radiance

See [16].

local volumetric rate of photon absorption (LVRPA), $L_{\mathrm{p}, \lambda}^{\mathrm{a}}(x, t)$

absorbed local spectral photon flux density

See [16].

\section{LVREA}

Abbreviation of local volumetric rate of energy absorption.

\section{LVRPA}

Abbreviation of local volumetric rate of photon absorption.

\section{mass transfer}

Mass diffusion or convection, arising from differences in concentration or in electrical potential at the initial and final locations under consideration.

Note 1: This is particularly significant in heterogeneous photocatalysis when the photocatalyst is anchored on a support and the resulting kinetics of the photoprocess may be masstransfer limited, as well as in large agglomerates of solid photocatalyst particles.

Note 2: In solid suspended catalyst it can be equally observed due to (a) the irreducible non-uniformities in the existing radiation field or (b) the use of very high fluence rates that turns the photocatalytic reaction rate independent of the absorbed (spectral) radiant power density. In the latter case (b) limitations other than mass transfer, e.g., light saturation, may be the reason for the independence of the photocatalysis rate on the absorbed radiation power.

See [8].

maximal photoadsorption capacity, $\theta_{\mathrm{m}}$

photoadsorption capacity

maximal photoadsorption stoichiometry

photoadsorption capacity

\section{memory effect}

Coekelbergs-Solonitsyn effect

Adsorption at the surface of a pre-irradiated adsorbent (photocatalyst) occurring in the dark after irradiation is terminated.

Note: The memory effect can be quantified by the post-adsorption memory coefficient, $K(t)$ :

$$
K(t)=\frac{N_{\text {post }}(t)}{N_{\text {phot }}(t)}
$$


where $N_{\text {post }}(t)$ is the number of entities (molecules, atoms, or ions) adsorbed after termination of irradiation time, $t$, and $N_{\text {phot }}(t)$ is the number of photoadsorbed entities during the irradiation time.

See Coekelbergs effect, Solonitsyn effect [27,44].

\section{mineralisation}

Complete conversion of an organic compound into carbon dioxide, water, and other simple inorganic compounds, depending on the hetero-atoms in the compound.

See photomineralisation.

\section{natural photosynthesis}

Production of complex organic substances by higher plants, algae and photosynthesising bacteria from simple compounds (e.g., carbon dioxide and water, hydrogen sulfide, etc.) using ultraviolet, visible, or infrared radiation energy absorbed by, e.g., chlorophyll, chlorophyll-protein complexes, and natural chromoproteins.

See also artificial photosynthesis, photosynthesis.

\section{OHP}

Abbreviation of $\underline{\text { outer }} \underline{\text { Helmholtz }}$ plane.

\section{optical thickness}

attenuance (napierian, $D_{\mathrm{e}}$ )

\section{outer Helmholtz plane (OHP)}

Locus of centres of nearest solvated ions non-specifically adsorbed.

Note: The layer between the inner Helmholtz plane (IHP) and the outer Helmholtz plane (OHP) is called the outer Helmholtz layer.

See inner Helmholtz plane [8,30].

\section{out-scattering}

scattering out (out of the beam under consideration)

Physical process by which the incoming electromagnetic radiation is forced to deviate from a straight trajectory by non-uniformities in the medium.

Note 1: Physical phenomenon taking into account scattered radiative energy from the rays in the direction $\Omega$ considered in the radiative transfer equation (RTE) into any direction $\Omega^{\prime}$.

Note 2: Mathematical definition: $\mathrm{Sc}_{\text {out }}=\sigma_{\lambda}(\boldsymbol{x}, t) L_{\Omega, \lambda}(\boldsymbol{x}, t)$ where $\boldsymbol{x}$ is the coordinate in a threedimensional space in the path of the beam, $t$ is the time coordinate, $\sigma_{\lambda}(x, t)$ is the spaceand time-dependent linear napierian scattering coefficient, $L_{\Omega, \lambda}(x, t)$ is the incident space- and time-dependent radiance in the direction $\Omega$.

$\mathrm{SI}$ unit is $\mathrm{W} \cdot \mathrm{sr}^{-1} \cdot \mathrm{m}^{-4}$. Common unit is $\mathrm{W} \cdot \mathrm{sr}^{-1} \cdot \mathrm{cm}^{-3} \cdot \mathrm{nm}^{-1}$.

Note 3: The mathematical definition in photon terms is: $\operatorname{Sc}_{\text {out }}=\sigma_{\lambda}(\boldsymbol{x}, t) L_{\mathrm{p}, \Omega, \lambda}(\boldsymbol{x}, t)$ with $L_{\mathrm{p}, \Omega, \lambda}(\boldsymbol{x}, t)$ the incident space- and time-dependent photon radiance. SI unit is $\mathrm{s}^{-1} \cdot \mathrm{sr}^{-1} \cdot \mathrm{m}^{-4}$ in photon number basis and $\mathrm{mol} \cdot \mathrm{s}^{-1} \cdot \mathrm{sr}^{-1} \cdot \mathrm{m}^{-4}$ in chemical amount basis. 
Common unit in photon number basis is $\mathrm{s}^{-1} \cdot \mathrm{sr}^{-1} \cdot \mathrm{cm}^{-3} \cdot \mathrm{nm}^{-1}$ and einstein $\cdot \mathrm{s}^{-1} \cdot \mathrm{sr}^{-1} \cdot \mathrm{cm}^{-3} \cdot \mathrm{nm}^{-1}$ in chemical amount basis.

See in-scattering, radiative transfer equation $[16,41]$.

$\boldsymbol{P}_{\mathrm{AE}}$

Photoadsorption efficiency

\section{PCA}

Abbreviation of photocatalytic activity

PCO

Abbreviation of photocatalytic oxidation

PE

Acronym for photonic efficiency

\section{PEC}

Acronym for photoelectrohemistry

phase function (for scattering), $B(\lambda)$

Distribution function representing the fraction of photons scattered from any direction $\Omega^{\prime}$ into the direction $\Omega$ under consideration in the radiative transfer equation (RTE). Dimension one.

See $[40,45]$.

\section{phonon}

Elementary excitation in the quantum mechanical treatment of vibrations in a crystal lattice. An energy bundle that behaves as a particle of energy $h v$, with $v$ the vibration frequency and $h$ the Planck constant.

Note 1: A phonon can be considered as an acoustic mode of thermal vibration of a crystal lattice (or liquid helium II).

Note 2: Every harmonic vibration can be decomposed in phonons, which are the elementary vibrations. The total number of phonons in a system that has internal vibrations (e.g., a crystal) is related to the temperature of the system.

Note 3: The concept of phonons provides a simplification in the theories of thermal and electrical conduction in solids.

Note 4: For example, interactions between phonons and electrons are thought to be responsible for such phenomena as "superconductivity".

See [8].

\section{photoadsorbate}

Entity (molecule, atom, or ion) bound to the surface of a solid (semiconductor or insulator) particle (typically to a photoadsorption centre) as the result of photoexcitation of either the solid or the entity (molecule, atom, or ion). 
Note: When photoadsorption is a step in a photocatalytic reaction, the photoadsorbed entity may be considered an intermediate in the given reaction.

\section{photoadsorbed species}

photoadsorbate

\section{photoadsorption}

photosorption

Adsorption, typically chemisorption, initiated by ultraviolet, visible, or infrared radiation absorbed either by the adsorbate or by the adsorbent.

Note: Photoadsorption is often considered as the primary chemical step in a heterogeneous photocatalytic reaction. There are, however, many cases in which the photocatalysed reaction is not initiated by adsorption onto the photocatalyst surface. Such is the case, e.g., of the photocatalysed oxidation of chlorinated hydrocarbons [46].

See $[8]$.

photoadsorption area number, $\gamma^{\mathrm{s}}$

Maximum amount of substance photoadsorbed per area on the uniformly irradiated surface of an adsorbent.

Note 1: Photoadsorption area number should be preferred to photoadsorption capacity (the original name given to this term [35]), since the latter term is reserved for the fractional number, referring to the number of regular sites available for photoadsorption.

Note 2: Mathematical expression, $\gamma^{\mathrm{s}}=N_{\max } / S$, where $N_{\max }$ is the maximal number of entities or moles photoadsorbed and $S$ the total surface area. SI unit is $\mathrm{m}^{-2}$ or $\mathrm{mol} \cdot \mathrm{m}^{-2}$. Common unit is $\mathrm{cm}^{-2}$ or $\mathrm{mol} \cdot \mathrm{cm}^{-2}$. Experimental values of $\gamma^{\mathrm{s}}$ in entities are in the range $10^{10}-10^{12} \mathrm{~cm}^{-2}$.

Note 3: The quantity $N_{\max }$ can be obtained by approximation from photoadsorption kinetics $N(t)$ on a uniformly irradiated surface provided that

(i) the powdered sample is uniformly irradiated. Then, the area of the irradiated surface is $S=m a^{\mathrm{s}}$, where $m$ is the mass of the sample and $a^{\mathrm{s}}$ is the specific surface area $\left(\mathrm{m}^{2} \cdot \mathrm{kg}^{-1}\right)$ of a given adsorbent, or

(ii) the efficiency of desorption of the adsorbed species (via a physical process) is negligible. Then the number of photocatalytic centres can be estimated from the photoadsorption area number, $\gamma^{\mathrm{s}}$, to determine TON and TOF for a given photocatalytic reaction.

See also photoadsorption capacity, photoadsorption fraction.

\section{photoadsorption capacity, $\theta_{\max }$}

Maximal number of entities (molecules, atoms, or ions) per regular surface site that can be photoadsorbed at a uniformly irradiated surface of an adsorbent,

$$
\theta_{\max }=N_{\max } / N^{\mathrm{ss}}
$$

where $N_{\max }$ is the maximal number of entities photoadsorbed and $N^{\mathrm{ss}}$ is the number of surface sites (mainly regular surface sites, i.e., regular lattice ions or atoms; typically, $N^{\mathrm{ss}} \mathrm{ca} .10^{15} \mathrm{~cm}^{-2}$ ). 
Note 1: $\theta_{\max }$ is a quantity of dimension one (a fractional number). $\theta_{\max }$ is about $10^{-5}-10^{-3}$.

Note 2: This term has been often called maximal photoadsorption capacity. This denomination is redundant, because capacity already refers to the maximal possible value of a property.

See dynamic photoadsorption process, photoadsorption fraction, turnover frequency, turnover number.

\section{photoadsorption centre}

Surface site or defect in its active state after photoexcitation capable of photoadsorbing species by a chemical interaction with the adsorbate entities (molecules, atoms, or ions).

See also active state of a surface photoadsorption centre.

\section{photoadsorption efficiency, $P_{\mathrm{AE}}$}

Ratio of the maximal (typically the initial) rate of photoadsorption of entities B (molecules, atoms, or ions) to the integrated incident (superscript 0 , zero) photon flux, $\int_{\lambda_{1}}^{\lambda_{2}} q_{\mathrm{p}, \lambda}^{0} \mathrm{~d} \lambda=q_{\mathrm{p}}^{0}=\mathrm{d} N_{\mathrm{p}}^{0} / \mathrm{d} t$, in the wavelength range $\lambda_{1}-\lambda_{2}$. Both rates should be measured under otherwise identical conditions (preferably as initial rates) in order to avoid concentration and/or surface area effects.

Note 1: Mathematical expression:

$$
P_{\mathrm{AE}}=\frac{\mathrm{d} N_{\mathrm{B}} / \mathrm{d} t}{\int_{\lambda_{1}}^{\lambda_{2}} q_{\mathrm{p}, \lambda}^{0} \mathrm{~d} \lambda} \text { for } t \rightarrow 0
$$

where $\mathrm{d} N_{\mathrm{B}} / \mathrm{d} t$ is the rate of adsorption of entities (the initial quasi-stationary rate of photoadsorption).

Note 2: The initial rate of photoadsorption in SI units is $\mathrm{d} p / \mathrm{d} t\left(\mathrm{~Pa} \cdot \mathrm{s}^{-1}\right)$ for gas/solid systems, with $p$ the pressure and in SI-coherent units $\mathrm{d} c / \mathrm{d} t\left(\mathrm{~mol} \cdot \mathrm{L}^{-3} \cdot \mathrm{s}^{-1}\right)$ for liquid/solid systems, with $c$ the amount concentration of the adsorbate in solution.

Note 3: For $P_{\mathrm{AE}}$ determined under first-order conditions on photon flux and zero-order on concentration (or pressure), it corresponds to the efficiency of formation of the active photoadsorption centre.

See [4].

photoadsorption fraction, $\theta_{\mathrm{ph}}$

Number of entities (atoms, molecules or ions) photoadsorbed per regular surface site at a uniformly irradiated surface of an adsorbent.

Note 1: Mathematical expression: $\theta_{\mathrm{ph}}=N / N^{\mathrm{ss}}$ with $N$ the number of entities photoadsorbed and $N^{\text {ss }}$ the number of surface sites (mainly regular surface sites, i.e., regular lattice ions or atoms; typically, $N^{\mathrm{ss}} \mathrm{ca} .10^{15} \mathrm{~cm}^{-2}$ ). $\theta_{\mathrm{ph}}$ is a quantity of dimension one (it is a fractional number).

Note 2: $\theta_{\mathrm{ph}}$ has been called photoadsorption number; fraction is preferred. It should not be called capacity, since the term capacity should be reserved for the maximal adsorption number. 
Note 3: The photoadsortion fraction, $\theta_{\mathrm{ph}}$, is smaller than the photoadsorption capacity, $\theta_{\max }$ (i.e., $\theta_{\mathrm{ph}}<\theta_{\mathrm{max}}$ ).

See photoadsorption area number, photoadsorption capacity.

\section{photoadsorption photonic yield}

Ratio of the maximal (typically the initial) rate of photoadsorption of entities B (molecules, atoms, or

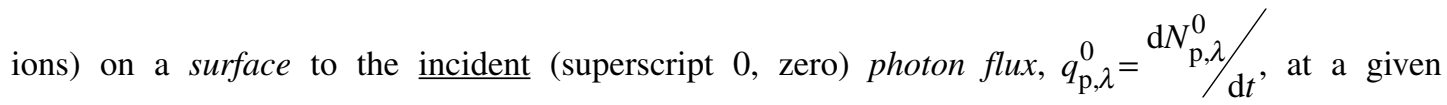
wavelength. Both rates should be measured under otherwise identical conditions (preferably as initial rates) in order to avoid concentration and/or surface area effects.

Note 1: Mathematical expression: $\frac{\mathrm{d} N_{\mathrm{B}} / \mathrm{d} t}{q_{\mathrm{p}, \lambda}^{0}}$ for $t \rightarrow 0$.

Note 2: Yield refers to the use of monochromatic irradiation, whereas efficiency is reserved for irradiation within a wavelength range. Photonic refers to incident radiation.

Compare with photoadsorption efficiency, photoadsorption quantum yield.

photoadsorption quantum yield, $\Phi_{\text {ads }}$

Ratio of the number of photoadsorbed entities B (molecules, atoms, or ions) on a surface to the number of photons of ultraviolet, visible, or infrared radiation (at a given wavelength $\lambda$ ) absorbed by the system (typically by the adsorbent) during the initial stage of irradiation.

Note: $\quad$ Mathematical expression: $\Phi_{\mathrm{ads}}=\frac{\mathrm{d} N_{\mathrm{B}} / \mathrm{d} t}{\mathrm{~d} N_{\mathrm{p}, \lambda} / \mathrm{d} t}$, for $t \rightarrow 0$, i.e., the ratio of the initial rates of photoadsorption and monochromatic photons absorbed, with $N_{\mathrm{B}}$ the number of entities adsorbed and $N_{\mathrm{p}, \lambda}$ the number of photons of wavelength $\lambda$ absorbed. The photoadsorption quantum yield has been called $K_{\mathrm{a}}$ in [47].

See the initial section Specific problems related to heterogeneous photocatalysis for a discussion of the problems related to the proper determination of quantum yields in heterogeneous photocatalysis.

Compare with photoadsorption efficiency, photoadsorption photonic yield.

See [47].

photoassisted catalysis

Catalytic transformations taking place under the action of ultraviolet, visible, or infrared radiation.

Note: The use of this term is recommended only for situations when it is still not known whether the ultraviolet, visible, or infrared radiation absorbing substance is a reactant species or a photocatalyst particle.

See also catalysed photochemical reaction, photocatalysis.

\section{photocarrier}

Nonequilibrium free electron or free hole generated in a solid by ultraviolet, visible, or infrared absorption and occupying energy levels in the conduction band or the valence band, respectively. 
Note: Electrons occupying energy levels near the bottom of the conduction band and holes occupying energy levels near the top of the valence band (thermalised carriers) are considered as quasi-chemical intermediates. Nonthermalised (hot) carriers can also be involved in photocatalysed reactions.

See charge carrier.

\section{photocatalysis}

Change in the rate of a chemical reaction or its initiation under the action of ultraviolet, visible, or infrared radiation in the presence of a substance — the photocatalyst - that absorbs light and is involved in the chemical transformation of the reaction partners.

See also photoassisted catalysis [4,7].

\section{photocatalyst}

Substance able to produce, by absorption of ultraviolet, visible, or infrared radiation, chemical transformations of the reaction partners, repeatedly coming with them into intermediate chemical interactions and regenerating its chemical composition after each cycle of such interactions.

See also photocatalysis.

\section{photocatalytic active centre}

Surface centre, i.e., either a surface defect or a regular surface site, at which a chemical transformation takes place after converting the centre into an active state by a photophysical process, e.g., by photoexcitation.

Note 1: The number of photoactive centres is very often difficult to establish, particularly on solid surfaces and in suspended particles.

Note 2: When the density of the active centres is unknown, the reaction rates may be related to the $\operatorname{BET}\left(\mathrm{N}_{2}\right)$ area with the understanding that the parameter or property of the system will be a lower limit of the actual value, because the surface determined by $\mathrm{N}_{2}$ adsorption will be larger than that reached by the radiation. See Note 2 under the definition of BET.

Note 3: The photophysical process may include one or several processes such as direct excitation of the photocatalytic centre via absorption of ultraviolet, visible, or infrared radiation, via photocarrier trapping, via interaction with excitons, or via energy and/or electron transfer from a photoexcited reagent or intermediate.

See $\operatorname{BET}\left(N_{2}\right)$, catalytic site.

\section{photocatalytic activity (PCA)}

Number of entities (product) formed in a photocatalytic process (alternatively the number of reactant entities disappearing) divided by the number of photons absorbed by the photocatalyst at one single wavelength or within a defined wavelength range. For one wavelength it has the same meaning as quantum yield, whereas within a defined wavelength range it has the same meaning as quantum efficiency.

Note: This quantity has a well-defined meaning only when the reaction conditions are also stated and the results reported in terms of the initial conditions.

See the section Considerations on the evaluation of quantum yields of heterogeneous photocatalytic reactions. 


\section{photocatalytic centre}

Surface centre, i.e., a surface defect or a regular surface site, in an active or electronically excited state at which a chemical transformation takes place.

See also catalytic site, photocatalytic active centre.

\section{photocatalytic efficiency}

Number of entities (product) formed (alternatively the number of reactant entities disappearing) divided by the number of ultraviolet, visible, or infrared photons incident (prior to absorption) on the system and in a wavelength range $\lambda_{1}-\lambda_{2}$.

Note 1: Quantity relevant in applications of photocatalysis where the interest is to assess an engineering efficiency of energy out vs. energy in. In this context, the quantity has a well-defined meaning only when the operating conditions are also stated and results reported in terms of the initial conditions.

Note 2: When using photon flux (number basis or chemical amount basis) the term photonic efficiency is preferred, as efficiency refers to excitation within a wavelength range, whereas for monochromatic irradiation (and incident photons) the term photonic yield should be used.

See photonic efficiency, photonic yield, quantum yield [7,8].

See the section Specific problems related to heterogeneous photocatalysis for a discussion of the difficulties associated with the determination of quantum yields in heterogeneous photocatalysis.

\section{photocatalytic oxidation (PCO)}

Commonly used to describe the oxidation of pollutants by the action of ultraviolet, visible, or infrared radiation and a catalyst.

Note: Often used to describe the oxidation of organic pollutants at low concentrations by UV-light irradiation of $\mathrm{TiO}_{2}$.

See [48].

photocatalytic site

photocatalytic active centre

See catalytic site.

\section{photocharge carrier}

photocarrier

See charge carrier.

\section{photochemistry}

See [7].

\section{photochemical reaction}

See [7]. 


\section{photoconductivity}

Increase in electric conductivity due to the photoproduction of charge carriers. See [8].

\section{photocorrosion}

Decomposition of the photocatalyst or of the electrodes as a result of a photoinduced reaction.

Note 1: Photocorrosion is one of the factors limiting the turnover number in heterogeneous photocatalysis.

Note 2: Process taking place during photoelectrochemical etching.

\section{photocurrent}

Photogeneration and movement of charge between two electrodes in a photovoltaic cell or a photoelectrochemical cell as a result of photoprocesses induced by light absorption.

\section{photocurrent yield}

Quantum yield of charge photogeneration between the two electrodes of a photovoltaic cell or a photoelectrochemical cell.

See [8].

\section{photodegradation}

Photochemical transformation of a molecule into lower molecular mass fragments taking place in an oxidation or reduction process.

Note 1: The process usually implies the incorporation of other atoms, especially $\mathrm{O}$ atoms during the oxidation processes.

Note 2: This term is widely used in the oxidative or reductive destruction of pollutants by UVor visible-light-based processes.

See $[8,26]$.

\section{photodesorption}

Desorption induced by the absorption of ultraviolet, visible, or infrared radiation either by the adsorbate or by the adsorbent.

Note 1: Photodesorption can be a step in the overall mechanism in heterogeneous photocatalysis.

Note 2: Photodesorption is the inverse process of photoadsorption. For a particular system, both processes (or reactions) are induced by ultraviolet, visible, or infrared radiation of the same wavelength region.

See dynamic photoadsorption process.

[8].

\section{photodesorption quantum efficiency}

See Note 4 in photodesorption quantum yield. 


\section{photodesorption quantum yield, $\Phi_{\text {des }}$}

Number of entities evolving into the medium from a surface divided by the number of photons absorbed at a given wavelength by a solid or by an adsorbed entity treated as a surface extrinsic defect (centre).

Note 1: Mathematical expression of the integral photodesorption quantum yield:

$$
\Phi_{\mathrm{des}}=\frac{N_{\mathrm{des}}}{N_{\mathrm{p}, \lambda}}
$$

where $N_{\text {des }}$ is the number of species desorbed and $N_{\mathrm{p}, \lambda}$ the number of photons of a particular wavelength absorbed during a given irradiation time.

Note 2: The differential expression is: $\Phi_{\mathrm{des}}=\frac{\mathrm{d} N_{\mathrm{des}} / \mathrm{d} t}{q_{\mathrm{p}, \lambda}}$ where $\mathrm{d} N_{\mathrm{des}} / \mathrm{d} t$ is the initial (quasistationary) or maximal photodesorption rate and $q_{\mathrm{p}, \lambda}=\mathrm{d} N_{\mathrm{p}, \lambda} / \mathrm{d} t$ is the spectral photon flux absorbed by a solid or by an adsorbed entity treated as a surface extrinsic defect (centre).

Note 3: Should photoexcitation of a system be due to absorption by pre-adsorbed species (see extrinsic absorption), $\Phi_{\mathrm{des}}$ would not depend on the concentration of pre-adsorbed species.

Note 4: In practice, for polychromatic irradiation, integration is performed in the wavelength range used and the quantity should be called photodesorption quantum efficiency.

\section{photoelectrocatalysis}

Electrochemically assisted photocatalysis. The role of the photocatalyst is played by a photoelectrode, often a semiconductor.

Note: In photoelectrocatalytic transformations it is possible to identify an electrode and an electrolyte combination in the system along with an explicit, generally macroscopic, electrical circuit for the charge transfer and/or for applying an additional electric potential to the system. This is not so in photocatalysis by colloids or fine dispersions of semiconductors.

See, e.g., $[49,50]$.

\section{photoelectrochemical cell}

Electrochemical cell in which current and a voltage are simultaneously produced upon absorption of ultraviolet, visible, or infrared radiation by one or more of the electrodes.

Note: Usually at least one of the electrodes is a semiconductor.

See $[8,51]$.

\section{photoelectrochemical etching}

Dissolution of a semiconductor in an electrolytic solution upon exposure to ultraviolet, visible, or infrared radiation. Photocorrosion takes place.

Note: $\quad$ Method used in the photopatterning of semiconductor surfaces.

See [8]. 


\section{photoelectrochemical transformation}

photoelectrocatalysis

\section{photoelectrochemistry (PEC)}

Hybrid field of chemistry employing techniques that combine photochemical and electrochemical methods for the study of the oxidation-reduction chemistry of the ground or excited states of molecules or ions.

Note: In general, it is the chemistry resulting from the interaction of ultraviolet, visible, or infrared radiation with electrochemical systems.

See $[8,21,52,53]$.

\section{photoelectrode}

Electrode capable of initiating electrochemical transformations after absorbing ultraviolet, visible, or infrared radiation.

\section{photoelectrosynthesis}

Process by which ultraviolet, visible, or infrared radiation is absorbed by a semiconductor; electron transfer occurs at the interface between the semiconductor and a liquid and the output is chemical energy, i.e., radiant energy is stored as chemical energy.

See [52].

\section{photoexcitation}

Production of an excited state by the absorption of ultraviolet, visible, or infrared radiation.

See [8].

\section{photogalvanic cell}

Electrochemical cell in which current or voltage changes result from photochemically generated changes in the relative concentrations of reactants in a solution phase oxidation-reduction couple.

Compare with photovoltaic cell [8].

\section{photogenerated catalysis}

Initiation of chemical transformations through the photochemical formation of molecular species, which (once formed) are catalytically active even without the action of ultraviolet, visible, or infrared radiation.

\section{photogenerated charge carrier}

Charge carrying entity, especially an electron or a hole, in a semiconductor, produced by the absorption of ultraviolet, visible, or infrared radiation.

\section{photoinduced adsorption}

photoadsorption

See [8]. 
photoinduced catalysis

photogenerated catalysis

photoinitiated catalysis

photogenerated catalysis

\section{photoinitiation}

Photoproduction of a free radical, a hole-electron pair, or an ion capable of initiating a chain reaction such as, e.g., polymerisation, halogenation, and nitrosylation.

See photoinitiator [8].

\section{photoinitiator}

Agent initiating certain chemical transformations under the action of ultraviolet, visible, or infrared radiation and consumed therewith.

Note: A photocatalyst can also act as a photoinitiator that, however, will not be consumed.

\section{photolysis}

Bond cleavage induced by ultraviolet, visible, or infrared radiation.

Note: Term often used incorrectly to describe irradiation of a sample or chemical effect induced by light other than a bond cleavage, although in the combination flash photolysis this usage is accepted.

See [8].

\section{photomineralisation}

Complete photodegradation of an organic compound into carbon dioxide and water, and other simple inorganic compounds (depending on the hetero-atom in the compound) upon absorption of ultraviolet, visible, or infrared radiation by the photocatalyst or by the adsorbate.

See mineralisation.

photon flux, $q_{\mathrm{p}}, \Phi_{\mathrm{p}}$

Number of photons (quanta of radiation, $N_{\mathrm{p}}$ ) per time interval. SI unit is $\mathrm{s}^{-1}$.

Note 1: Mathematical definition: $q_{\mathrm{p}}=\mathrm{d} N_{\mathrm{p}} / \mathrm{d} t$. If the number of photons is constant over the time interval, then $q_{\mathrm{p}}=N_{\mathrm{p}} / t$.

Note 2: This quantity can be used on a chemical amount basis by dividing the photon flux, number basis, $q_{\mathrm{p}}$, by the Avogadro constant, the symbol then being $q_{n, \mathrm{p}}$, the name "photon flux, amount basis". SI unit is $\mathrm{mol} \cdot \mathrm{s}^{-1}$ and common unit is einstein $\cdot \mathrm{s}^{-1}$.

Note 3: Although the symbol recommended by CIE (Commission Internationale de L'éclairage) [12] is $\Phi_{\mathrm{p}}$, the symbol $q_{\mathrm{p}}$ is preferred since $\Phi$ is reserved for quantum yield.

Note 4: Term as defined in [12]. It is a term not in accordance with the usual definition of flux as defined in [9].

See spectral photon flux.

For related terms see $[7,8]$. 


\section{photon irradiance, $E_{\mathrm{p}}$}

Number of photons (quanta of radiation, $N_{\mathrm{p}}$ ) per time interval (photon flux), $q_{\mathrm{p}}$, incident from all upward directions on a small element of surface containing the point under consideration divided by the area of the element. SI unit is $\mathrm{m}^{-2} \cdot \mathrm{s}^{-1}$.

Note 1: Mathematical definition: $E_{\mathrm{p}}=\mathrm{d} q_{\mathrm{p}} / \mathrm{d} S$. If the photon flux is constant over the surface considered, then $E_{\mathrm{p}}=q_{\mathrm{p}} / S$.

Note 2: This term refers to a beam not scattered or reflected by the target or its surroundings. For a beam incident from all directions, photon fluence rate $\left(E_{\mathrm{p}, \mathrm{o}}\right)$ is the preferred term (subindex o).

Note 3: This quantity can be used on a chemical amount basis by dividing $E_{\mathrm{p}}$ by the Avogadro constant, the symbol then being $E_{n \text {,p }}$, the name "photon irradiance, amount basis". SI unit is $\mathrm{mol} \cdot \mathrm{m}^{-2} \cdot \mathrm{s}^{-1}$ and common unit is einstein $\cdot \mathrm{m}^{-2} \cdot \mathrm{s}^{-1}$.

See spectral photon irradiance.

For related terms, see [7,8].

\section{photon quantities}

Quantities characterising electromagnetic radiation in terms of the number of photons. The symbols of photon quantities may be distinguished by adding a subscript $\mathrm{p}$.

See [8].

photon radiance, $L_{\mathrm{p}, \Omega}$

Number of photons (quanta of radiation, $N_{\mathrm{p}}$ ) per time interval (photon flux), $q_{\mathrm{p}}$, leaving or passing through a small transparent element of surface in a given direction from the source about the solid angle $\Omega$, divided by the solid angle and by the orthogonally projected area of the element in a plane normal to the given beam direction, $\mathrm{d} S_{\perp}=\mathrm{d} S \cos \theta$, with $\theta$ the angle between the normal to the surface and the direction of the beam.

Note 1: Equivalent definition: Integral taken over the hemisphere visible from the given point, of the expression $L_{\mathrm{p}} \cos \theta \mathrm{d} \Omega$, with $L_{\mathrm{p}}$ the photon radiance at the given point in the various directions of the incident beam of solid angle $\Omega$ and $\theta$ the angle between any of these beams and the normal to the surface at the given point.

Note 2: Mathematical definition [9]:

$$
L_{\mathrm{p}, \Omega}=\lim _{(\Delta \Omega, \Delta S, \Delta t) \rightarrow 0} \frac{\Delta N_{\mathrm{P}}}{\Delta \Omega \Delta S \cos \theta \Delta t}
$$

for a divergent beam propagating in an elementary cone of the solid angle $\Omega$ containing the direction $\theta$. SI unit is $\mathrm{m}^{-2} \cdot \mathrm{s}^{-1} \cdot \mathrm{sr}^{-1}$.

Note 3: Photon radiance of a divergent beam is the quantity used when working in three-dimensional reaction spaces, in order to obtain useful intrinsic kinetic results for up-scaling calculations. The mathematical expression above takes into account scattering and has a local value at any time for any point inside the reaction space.

Note 4: For a parallel beam, it is the number of photons (quanta of radiation, $N_{\mathrm{p}}$ ) per time interval (photon flux), $q_{\mathrm{p}}$, leaving or passing through a small element of surface in a given direction from the source divided by the orthogonally projected area of the element in a plane normal to the given direction of the beam, $\theta$. Mathematical definition in this 
case: $L_{\mathrm{p}}=\mathrm{d} q_{\mathrm{p}} /(\mathrm{d} S \cos \theta)$. If $q_{\mathrm{p}}$ is constant over the surface area considered, $L_{\mathrm{p}}=$ $q_{\mathrm{p}} /(S \cos \theta)$, SI unit is $\mathrm{m}^{-2} \cdot \mathrm{s}^{-1}$.

Note 5: This quantity can be used on a chemical amount basis by dividing $L_{\mathrm{p}}$ by the Avogadro constant, the symbol then being $L_{n, \mathrm{p}}$, the name "photon radiance, amount basis". For a divergent beam, SI unit is $\mathrm{mol} \cdot \mathrm{m}^{-2} \cdot \mathrm{s}^{-1} \cdot \mathrm{sr}^{-1} ;$ common unit is einstein $\cdot \mathrm{m}^{-2} \cdot \mathrm{s}^{-1} \cdot \mathrm{sr}^{-1}$. For a parallel beam, SI unit is $\mathrm{mol} \cdot \mathrm{m}^{-2} \cdot \mathrm{s}^{-1}$; common unit is einstein $\cdot \mathrm{m}^{-2} \cdot \mathrm{s}^{-1}$.

Note 6: Photon radiance has been called flux density (of particles or photons) in radiation chemistry areas $[54,55]$.

See [7].

photonic efficiency, $\xi$

Ratio of the rate of the photoreaction measured for a specified time interval (usually the initial conditions) to the rate of incident photons within a defined wavelength interval inside the irradiation window of the reactor.

See [56].

Note 1: A quantity called formal quantum efficiency (FQE) is sometimes used as synonymous for photonic efficiency and calculated from the known spectral distribution of the excitation source and the known absorption spectrum of the reaction system. It is calculated as the ratio between the rate of reaction and the incident photon flux [56].

Note 2: When monochromatic radiation is used, formal quantum efficiency is $\xi$; otherwise formal quantum efficiency $\neq \xi$.

Note 3: Mathematical definition: $\xi=\frac{\mathrm{d} n / \mathrm{d} t}{\int_{\lambda_{1}}^{\lambda_{2}} q_{n, \mathrm{p}, \lambda}^{0} \mathrm{~d} \lambda}$ when using the incident spectral photon flux, chemical amount basis, $q_{n, \mathrm{p}, \lambda}^{0}$, with $n$ (mol) the amount of substance produced or consumed, and $\xi=\frac{\mathrm{d} N / \mathrm{d} t}{\int_{\lambda_{1}}^{\lambda_{2}} q_{\mathrm{p}, \lambda}^{0} \mathrm{~d} \lambda}$ when using the incident spectral photon flux, number basis, $q_{\mathrm{p}, \lambda}^{0}$ with $N$ the number of entities produced or consumed. The superscript 0 (zero) emphasises the fact that the incident number of photons (prior to absorption) is used.

See also photocatalytic efficiency, photonic yield, quantum efficiency, relative photonic efficiency.

\section{photonic yield}

Ratio of the rate of the photoreaction measured for a specified time interval (usually the initial conditions) to the rate of incident photons of monochromatic light inside the irradiation window of the reactor.

Note: Mathematical definition: $\frac{\mathrm{d} n / \mathrm{d} t}{q_{n, \mathrm{p}, \lambda}^{0}}$ when using the incident spectral photon flux, chemical amount basis, $q_{n, \mathrm{p}, \lambda}^{0}$, with $n$ (mol) the amount of substance produced or consumed, and $\frac{\mathrm{d} N / \mathrm{d} t}{q_{\mathrm{p}, \lambda}^{0}}$ when using the incident spectral photon flux, number basis, $q_{\mathrm{p}, \lambda}^{0}$, with 
$N$ the number of entities produced or consumed. The superscript 0 (zero) emphasises the fact that the incident number of photons (prior to absorption) is used.

See photonic efficiency, quantum efficiency, quantum yield.

\section{photonic yield spectrum}

Plot of the photonic yield of a given photoprocess, measured at the same photon flux of incident (prior to absorption) light, as a function of a measure of the energy of the photons, i.e., for monochromatic irradiation.

Note 1: In practice, the photonic yield spectrum is obtained by normalising the rate of the given process to a certain number of incident photons provided that the photoresponse is linear on the incident photon fluence.

Note 2: Should the fluence dependence of the photoresponse be nonlinear (as is often the case in catalysed photoresponses), a plot of the photoresponse vs. fluence should be made at several wavelengths and a standard response should be chosen (e.g., two-lg reduction). A plot of the inverse of the "standard response" level as a function of wavelength is then the action spectrum of the photoresponse.

Note 3: Note that in photocatalysis the term yield is reserved for monochromatic irradiation, whereas the term efficiency is used for irradiation within a wavelength range. This is different from the use of efficiency as defined in [7].

See action spectrum.

\section{photooxidation}

See [8].

\section{photooxygenation}

See [7].

\section{photoradiation catalysis}

Radiation catalysis under conditions not allowing a distinction between the effect of ultraviolet, visible, or infrared and of ionising radiation as well as the case in which the ionising radiation alone generates emission of chemically active light (e.g., the "Cherenkov" light emission).

\section{photoreaction}

See [7].

\section{photoreduction}

See [8].

\section{photosensitisation}

sensitisation

Photochemical or photophysical alteration occurring in one molecular entity as a result of initial absorption of radiation by another molecular entity called a photosensitiser.

See [8]. 


\section{photosensitised reaction}

Reaction initiated by photosensitisation.

See catalysed photochemical reaction [7].

\section{photosensitiser}

See [8].

\section{photosorption}

photoadsorption

Photoadsorption is the preferred term.

\section{photostationary state}

See [8].

photosynthesis (in relation to nonbiological systems)

Synthesis of molecular species by the action of ultraviolet, visible, or infrared radiation.

See artificial photosynthesis, natural photosynthesis.

\section{photothermocatalysis}

Catalysis occurring by the combined action of electronic excitation due to absorption of ultraviolet or visible radiation and thermal energy (infrared radiation or exothermic reactions) including catalytic phenomena.

Note: This often occurs upon exposure of a heterogeneous photocatalyst to infrared-light, laser emission, or solar radiation.

\section{photovoltaic cell}

Solid-state device, usually based on a semiconductor (e.g., silicon), which absorbs photons with energies higher than or equal to the bandgap energy and simultaneously produces electric power.

Compare with photogalvanic cell.

See [8].

\section{physical pathway}

Set of physical processes leading to the relaxation of the electronically excited system without chemical transformations.

Note: Examples are radiative and nonradiative relaxations in molecules and surface complexes from the excited state to the ground state, recombination and trapping of free charge carriers and recombination decay of trapped carriers (particularly, recombination decay of carriers trapped by surface-active centres).

\section{physisorption}

Adsorption of an entity (molecules, atoms, or ions) onto a surface by way of a physical interaction (usually van der Waals forces, dipolar interactions, etc.) between the surface and the species. The Gibbs energy of interaction is relatively low in these cases.

Note: An example is the physisorption of a hydrocarbon onto a metal-oxide particle surface. See chemisorption $[8,9]$. 
point of zero charge (PZC)

Condition for which the electrical charge on a surface of a material is zero, i.e., the surface is electrically neutral.

Note: $\quad$ PZC will be affected by surface adsorbed species.

See [8].

post-adsorption

See Coekelbergs effect, memory effect, Solonitsyn effect [44].

post-adsorption memory coefficient, $K(t)$

See memory effect [44].

primary photochemical process (primary photoreaction)

Elementary chemical process undergone by an electronically excited (molecular) entity and yielding a primary photoproduct.

See primary photoprocess [8].

primary photoprocess

The term primary photoprocess for a photophysical process could lead to inconsistencies and its use is discouraged.

See primary photochemical process [8].

primary (photo)product

First observable chemical entity produced in the primary photochemical process and that is chemically different from the reactant.

See primary photochemical process, primary photoprocess [8].

\section{PSA}

Abbreviation of photostimulated adsorption.

PZC

Abbreviation of point of zero charge.

\section{quantum efficiency}

Rate of a given photophysical or photochemical process divided by the total absorbed photon flux.

Note 1: This applies especially for polychromatic excitation, i.e., within a wavelength range.

Note 2: Mathematical expression for homogeneous systems:

$$
\Phi(\Delta \lambda)=\frac{\int_{\lambda_{1}}^{\lambda_{2}} \frac{\mathrm{d} x(\lambda)}{\mathrm{d} t} \mathrm{~d} \lambda}{\int_{\lambda_{1}}^{\lambda_{2}} q_{n, \mathrm{p}, \lambda}^{0}\left[1-10^{-A(\lambda)}\right] \mathrm{d} \lambda}
$$

where $\mathrm{d} x(\lambda) / \mathrm{d} t$ is the rate of change of a quantity (spectral or any other property) that measures the progress of a catalytic reaction, and $q_{n, \mathrm{p}, \lambda}^{0}$ the amount of photons (mol or 
its equivalent non-SI unit einstein) incident (prior to absorption, superscript 0 , zero) per time interval of the particular wavelength used (spectral photon flux, amount basis). $A(\lambda)$ is the decadic absorbance at the excitation wavelength.

Note 3: For a divergent beam

$$
\Phi(\Delta \lambda)=\frac{\int_{\lambda_{1}}^{\lambda_{2}}[\mathrm{~d} x(\lambda) / \mathrm{d} t] \mathrm{d} \lambda}{\int_{\lambda_{1}}^{\lambda_{2}}\left[\int_{V} \alpha_{\lambda}(\boldsymbol{x}, t) E_{n, \mathrm{p}, \lambda_{, 0}}(\boldsymbol{x}, t) \mathrm{d} V\right] \mathrm{d} \lambda}
$$

where the integral over the volume $V$ in the denominator is the total absorbed spectral photon flux, chemical amount basis.

Note 4: In homogeneous photochemistry, and when the quantum yield is independent of excitation wavelength in the spectral region used, quantum efficiency is identical to quantum yield [8], which is always referred to monochromatic excitation.

Note 5: In heterogeneous photocatalysis, the photonic efficiency $(\xi)$ is commonly used, i.e., per photon incident in the system within a specified wavelength range.

See efficiency, formal quantum efficiency, photonic efficiency, photonic yield, quantum yield [7].

quantum yield, $\Phi$

Number of defined events, occurring per photon absorbed by the system at a specified wavelength.

Note 1: The integral quantum yield $\Phi(\lambda)$ is the number of events divided by the number of photons absorbed at a specified wavelength.

Note 2: For a photochemical reaction $\Phi(\lambda)$ is the amount of reactant consumed or product formed divided by the amount of photons absorbed [57].

Note 3: The differential quantum yield for a homogeneous system is (in terms of rates),

$$
\Phi(\lambda)=\frac{\mathrm{d} x / \mathrm{d} t}{q_{n, \mathrm{p}, \lambda}^{0}\left[1-10^{-A}\right]}
$$

where $\mathrm{d} x / \mathrm{d} t$ is the rate of change of a quantity $x$ that measures the progress of a reaction and $q_{n, \mathrm{p}, \lambda}^{0}$ is the spectral amount of photons (mol or its equivalent non SI-unit einstein) incident per time interval (spectral photon flux, amount basis). $A(\lambda)$ is the decadic absorbance at the excitation wavelength.

Note 4: When $x$ in the equation for the differential quantum yield (see above) is either a number concentration, or an amount concentration, it is convenient to use in the denominator the rate of absorbed spectral photons per volume (absorbed photon flux density) number basis, or chemical amount basis, respectively.

Note 5: $\Phi$ can be used for photophysical processes or photochemical reactions.

Note 6: Strictly, the term quantum yield applies only for monochromatic excitation.

Note 7: For a photocatalytic reaction in a large photoreactor of volume $V$, the average differential quantum yield is

$$
\Phi(\lambda)=\frac{\mathrm{d} x / \mathrm{d} t}{\left\langle L_{\mathrm{p}, \lambda}^{\mathrm{a}}(t)\right\rangle_{V}}
$$


where $\left\langle L_{\mathrm{p}, \lambda}^{\mathrm{a}}(t)\right\rangle_{V}$ is the volume-averaged absorbed (spectral) photon flux density and $\mathrm{d} x / \mathrm{d} t$ is the time evolution of the amount concentration of the compound formed (or reactant consumed). This concept becomes necessary in cases of large photoreactors with heterogeneous distribution of radiation and high radiation scattering. In view of the size of the large photoreactors, actinometry is not possible in most of these cases and calculations with $\left\langle L_{\mathrm{p}, \lambda}^{\mathrm{a}}(t)\right\rangle_{V}$ are needed [16,41].

Compare with quantum efficiency.

See efficiency, photonic efficiency, photonic yield [8].

\section{quencher}

Molecular entity that deactivates (quenches) an excited state of another entity, either by energy transfer, electron transfer, or by a chemical mechanism.

See quenching [8].

\section{quenching}

Deactivation of an excited molecular entity intermolecularly by an external environmental influence (such as a quencher) or intramolecularly by a substituent through a nonradiative process.

Note 1: When the external environmental influence (quencher) interferes with the decay of the excited state after its formation, the process is referred to as dynamic quenching. Common mechanisms include energy transfer, charge transfer, etc.

Note 2: When the environmental influence inhibits the excited state formation, the process is referred to as static quenching.

See [8].

\section{radiance}

Radiant power, $P$, leaving or passing through a small transparent element of surface in a given direction from the source about the solid angle $\Omega$, divided by the solid angle and by the orthogonally projected area of the element in a plane normal to the given beam direction, $\mathrm{d} S_{\perp}=\mathrm{d} S \cos \theta$.

Note 1: Mathematical definition [9]:

$$
L_{\Omega}=\lim _{(\Delta \Omega, \Delta S, \Delta t) \rightarrow 0} \frac{\Delta P}{\Delta \Omega \Delta S \cos \theta}
$$

for a divergent beam propagating in an elementary cone of the solid angle $\Omega$ containing the direction $\theta$. SI unit is $\mathrm{W} \cdot \mathrm{m}^{-2} \cdot \mathrm{sr}^{-1}$.

Note 2: For a parallel beam it is the radiant power, $P$, of all wavelengths leaving or passing through a small element of surface in a given direction from the source divided by the orthogonally projected area of the element in a plane normal to the given direction of the beam, $\theta$. Mathematical definition in this case: $(\mathrm{d} P /(\mathrm{d} S \cos \theta)$. If the radiant power is constant over the surface area considered, $L=P /(S \cos \theta)$. SI unit is $\mathrm{W} \cdot \mathrm{m}^{-2}$.

Note 3: Equivalent to $L=\int_{\lambda} L_{\lambda} \mathrm{d} \lambda$, where $L_{\lambda}$ is the spectral radiance at wavelength $\lambda$. See [8]. 


\section{radiant dose}

Radiant energy ( $Q$, SI unit is $\mathbf{J}$ ) or amount of photons (in mol or the non-SI unit einstein) absorbed per area or volume by an object irradiated during a given exposure time.

radiant energy, $Q, \mathrm{~W}$

Total energy emitted, transferred or received as radiation of all wavelengths in a defined period of time. SI unit is J.

Note 1: Mathematical definition: $Q=\int_{\lambda} Q_{\lambda} \mathrm{d} \lambda$.

Note 2: If the radiant power $P$ is constant over the time interval, then $Q=P t$.

See $[8,12]$.

radiant energy fluence

fluence

radiant (energy) flux, $P, \Phi$

This obsolete term is not recommended.

In photochemistry, radiant power, $P$ is adopted. SI unit is $\mathrm{W}$.

Note: $\quad P$ is preferred, because in photochemistry $\Phi$ is reserved for quantum yield.

Related terms: photon flux, radiant energy.

See $[8,12,58]$.

\section{radiant intensity, $I$}

intensity

Radiant power, $P$, at all wavelengths per solid angle, $\Omega$. The radiant power emitted in a given direction by a source or an element of the source in a small cone containing the given direction divided by the solid angle of the cone. SI unit is $\mathrm{W} \cdot \mathrm{sr}^{-1}$.

Note 1: Mathematical definition: $I=\mathrm{d} P / \mathrm{d} \Omega$. If the radiant power is constant over the solid angle considered, then $I=P / \Omega$.

Note 2: Equivalent to $I=\int_{\lambda} I_{\lambda} \mathrm{d} \lambda$, where $I_{\lambda}$ is the spectral radiant intensity at wavelength $\lambda$.

Note 3: It is not recommended to abbreviate this term to just intensity because it is confusing. See $[8,12]$.

\section{radiant power, $P$}

Power emitted, transferred or received as radiation. SI unit is $\mathrm{J} \cdot \mathrm{s}^{-1}=\mathrm{W}$.

Note 1: Mathematical definition: $P=\mathrm{d} Q / \mathrm{d} t$. If the radiant energy, $Q$, is constant over the time interval, $P=Q / t$.

Note 2: In radiometry, flux $(\Phi)$ is used with the same units as $P$. The symbol $\Phi$ is reserved for quantum yield in photochemistry. Therefore, the use of flux $(\Phi)$ is not recommended.

See spectral radiant power $[8,9]$. 


\section{radiant quantities}

Quantities characterising electromagnetic radiation in terms of energy. The symbols of radiant quantities may be distinguished by adding a subscript e, for energy.

See [8].

\section{radiation}

Term embracing electromagnetic waves as well as fast-moving particles. In radioanalytical chemistry the term usually refers to radiation emitted during a nuclear process (e.g., radioactive decay, nuclear reaction, nuclear fission, accelerators).

Note: In this Glossary, however, in general we refer to radiation as embracing electromagnetic waves from all wavelength and not fast-moving particles. For terms related to this area, see $[54,55,58]$.

See [8].

radiation-activation of a catalyst

radioactivation of a catalyst

Increasing of catalytic activity or alteration of selectivity of a substance after its exposure to highenergy radiation.

\section{radiation adsorption}

radiation-induced adsorption

radioadsorption

The word combination may be misleading. Usually radiation is absorbed [7].

\section{radiation catalysis}

Change in the rate of a chemical reaction under the action of ionising radiation in the presence of a radiation catalyst.

Note: When using ionising radiation, from vacuum ultraviolet to higher energy, the phenomenon of photocatalysis cannot be distinguished from the phenomenon of radiation catalysis. Due to the nonselective absorption of ionising radiation, excitation of all reaction partners (both the reactants and the reaction catalysts) is possible. Thus, the phenomena globally designated as radiation catalysis include both direct radiation-induced and catalysed processes.

\section{radiation catalyst}

Substance absorbing ionising radiation and able to produce chemical transformations of the reaction partners, repeatedly producing intermediate transformations and regenerating its chemical composition after each cycle of such interactions.

radiation-chemical yield, $G$ value

Number of entities of a product formed (alternatively, the number of entities of a reactant disappearing) generated per $100 \mathrm{eV}$ of energy absorbed from ionising radiation.

Note 1: Mathematical expression: $G=100\left(N_{\mathrm{B}} / Q\right)$ where $N_{\mathrm{B}}$ is the number of entities B formed (or disappeared) and $Q$ is the absorbed radiation energy in $\mathrm{eV}$. Unit is $(100 \mathrm{eV})^{-1}$. 
Note 2: In SI units it refers to the amount of substance in $\mu$ mol generated for each joule of radiation absorbed by the system. SI unit is $\mu \mathrm{mol} \cdot \mathrm{J}^{-1}$.

Note 3: It is convenient to use the amount of substance B in $10^{-7} \mathrm{~mol}$ of $\mathrm{B}$, generated for each joule of radiation absorbed on the system. By choosing this (submultiple of) SI coherent unit, a number almost identical to the $G$ value (within $4 \%$ ) in the obsolete unit $(100 \mathrm{eV})^{-1}$ is obtained, as $(100 \mathrm{eV})^{-1}=1.036 \times 10^{-7} \mathrm{~mol} \cdot \mathrm{J}^{-1}$.

Note 4: When the energy absorbed by the radiation catalyst cannot be calculated (as is often the case in radiation catalysis), the radiation chemical yield is calculated in terms of the energy absorbed by the system. In this case, it is an apparent yield.

See effective radiation-catalytic activity $[8,59,60]$.

\section{radiation desorption}

Desorption from solid or liquid interfaces induced by absorption of radiation by the solid or liquid adsorbent.

\section{radiation-generated catalysis}

Initiation of chemical transformations through the formation, by absorption of high-energy radiation, of substances that are catalytically active even in the absence of the radiation.

Compare with radiation catalysis.

\section{radiation-induced adsorption}

radioadsorption

radiation adsorption

Adsorption of a molecular entity at solid or liquid interfaces induced by absorption of high-energy radiation by the solid or the liquid adsorbent.

Note: The word combination radiation adsorption is misleading. Usually, radiation is absorbed.

\section{radiation-induced desorption}

radiation desorption

\section{radiation modification}

See radiation-activation of a catalyst.

\section{radiation sensitisation}

\section{radiosensitisation}

Chemical change or physical process occurring in one entity (e.g., biological molecules, cells, and living organisms) as a result of initial absorption of high-energy radiation by the medium in the presence of another entity called the radiation sensitiser.

Note: High-energy radiation is absorbed usually nonspecifically in the medium (e.g., solvent) and all chemical reactions arise from the products of this primary energy absorption.

See $[59,60]$. 
radiation sensitiser

radiosensitiser

Chemical substance that increases the radiation sensitivity of, in particular, biological molecules, cells and biological organisms.

See radiation sensitisation.

\section{radiation-stimulated diffusion}

radiostimulated diffusion

Increase of diffusion of an adsorbate under the action of ionising radiation absorbed by either the adsorbent and/or the adsorbate.

\section{radiation thermal catalysis}

radiothermal catalysis

Radiation catalysis under the action of large radiation fluxes that simultaneously heat the system as a consequence of absorption of energy from radiationless deactivation.

\section{radiative transfer equation (RTE)}

When a photon beam of radiance $L_{\lambda}$ and angular distribution $\Omega$ passes along a single direction of propagation through a medium, which is totally absorbing and/or scattering, i.e., the processes occurring are described by the following equation:

$$
\begin{aligned}
& \frac{\mathrm{d} L_{\Omega, \lambda}(\boldsymbol{x}, t)}{\mathrm{d} s}+\underbrace{\alpha_{\lambda}(\boldsymbol{x}, t) L_{\Omega, \lambda}(\boldsymbol{x}, t)}_{\text {absorption }}+\underbrace{\sigma_{\lambda}(\boldsymbol{x}, t) L_{\Omega, \lambda}(\boldsymbol{x}, t)}_{\text {out-scattering }}= \\
& \underbrace{j_{\lambda}(\boldsymbol{x}, t)}_{\text {emission }}+\underbrace{\frac{\sigma_{\lambda}(\boldsymbol{x}, t)}{4 \pi} \int_{4 \pi} B_{\lambda}\left(\Omega^{\prime} \rightarrow \Omega\right) L_{\Omega, \lambda}(\boldsymbol{x}, t) \mathrm{d} \Omega^{\prime}}_{\text {in-scattering }}
\end{aligned}
$$

where $\frac{\mathrm{d} L_{\Omega, \lambda}(\boldsymbol{x}, t)}{\mathrm{d} s}$ is the directional derivative along the direction $s$ at a point $\boldsymbol{x}$ in the reaction space of the incident radiance, $\alpha_{\lambda}(\boldsymbol{x}, t)$ is the space- and time-dependent spectral linear napierian absorption coefficient, $\sigma_{\lambda}(x, t)$ is the space- and time-dependent spectral linear napierian scattering coefficient, $j_{\lambda}(\boldsymbol{x}, t)$ represents the spontaneous and/or induced emission, and $B_{\lambda}$ is the phase function. All quantities are a function of time. The RTE is written for the direction $s$.

Note 1: Absorption and out-scattering reduce the radiance along the axis of propagation, whereas in-scattering and emission, increases it. Normally, emission can be safely neglected in photocatalysis.

Note 2: The RTE is a representation of the (radiation) energy conservation law and its solution in cases with complex geometry and in the presence of scattering remains a challenging issue.

Note 3: Back scattering and forward scattering are global effects derived from the integration of absorption, in-scattering, out-scattering, and emission.

See local differential absorption, in-scattering, out-scattering [40,61].

\section{radioactivation of a catalyst}

radiation-activation of a catalyst

(C) 2011, IUPAC

Pure Appl. Chem., Vol. 83, No. 4, pp. 931-1014, 2011 


\section{radioadsorption}

radiation-induced adsorption

\section{radiodesorption}

radiation-induced desorption

\section{radiolysis}

Bond cleavage induced by high-energy radiation.

Note: Term loosely used for any chemical and physical process brought about by high-energy radiation as well as to refer to the irradiation technique itself ("pulse radiolysis").

See $[8]$.

radiomodification of catalysts

radiation activation of catalysts

radiosensitisation

radiation sensitisation

radiosensitiser

radiation sensitiser

radiostimulated diffusion

radiation-stimulated diffusion

radiothermal catalysis

radiation thermal catalysis

rate of (photo)generation of (charge) carriers, $g$

Number of carriers (or electron-hole pairs, or excitons) produced per time interval and per volume of a solid photocatalyst particle due to the absorption of light.

Note 1: The rate of photogeneration in the bulk of optical uniform photocatalyst particles depends on the distance $(x)$ from the irradiated surface.

Note 2: The rate of photogeneration of carriers, $g(x)$, has the general mathematical expression:

$$
g(x)=\alpha(\lambda) E_{\mathrm{p}, \lambda}^{0}(1-\rho) \exp [-\alpha(\lambda) x]
$$

where $\alpha(\lambda)$ is the napierian (spectral) absorption coefficient [7], $E_{\mathrm{p}, \lambda}^{0}$ is the incident (superscript 0) spectral photon irradiance and $\rho$ the reflectance. SI unit of $g(x)$ is $\mathrm{m}^{-3} \cdot \mathrm{s}^{-1}$ for $E_{\mathrm{p}, \lambda}^{0}$ in photon number basis and $\mathrm{mol} \cdot \mathrm{m}^{-3} \cdot \mathrm{s}^{-1}$ for $E_{n, \mathrm{p}, \lambda}^{0}$ in chemical amount basis.

Note 3: $g(x)$ is related to the number of photogenerated electrons $\left(N_{\mathrm{e}}\right)$ and holes $\left(N_{\mathrm{h}}\right)$ as $N_{\mathrm{e}}=$ $g(x) \tau_{\mathrm{e}}$ and $N_{\mathrm{h}}=g(x) \tau_{\mathrm{h}}$, where $\tau_{\mathrm{e}}$ and $\tau_{\mathrm{h}}$ are the lifetimes of the free electrons and holes, respectively. 
Note 4: In kinetic studies of photocatalysed reactions, the rate of generation is typically considered as a constant $g=\alpha(\lambda) E_{\mathrm{p}, \lambda}^{0}$ (uniform generation), which corresponds to the socalled weak light absorption case $[\alpha(\lambda) x<<1]$.

Note 5: This term refers to charge carriers. The definition of carriers is different from that given in [8], which refers to mass carriers.

See rate of reaction in [8].

rate of surface recombination, $s$

Decay of carriers at the surface of a solid or interface between different phases, e.g., at the crystal surface.

Note: Inverse of the lifetime of free carriers in the bulk of the particle. The greater $s$ is, the smaller is the stationary concentration of photocarriers at the surface (and vice versa). SI unit is $\mathrm{s}^{-1}$.

See surface concentration of photocarrier, surface recombination.

\section{reactive oxygen species $(\mathrm{ROS})$}

ROS are oxygen-containing species. They include the highly reactive oxygen atoms, free radicals, singlet molecular dioxygen, hydrogen peroxide as well as other peroxides, both inorganic and organic. The radical-anion called superoxide and its conjugated acid (hydroperoxyl radical) are poorly reactive with organic compounds in aqueous solution because they disproportionate very efficiently with each other to yield the highly reactive hydrogen peroxide and $\mathrm{O}_{2}[62]$.

Note 1: During a photocatalytic process, ROS are formed by the absorption of ultraviolet, visible, or infrared radiation by a semiconductor or insulator followed by oxidation of the surface hydroxyl groups or water by the valence band holes and/or by the reduction of surface chemisorbed molecular oxygen by the conduction band electrons [e.g., HO;, $\mathrm{O}_{2}{ }^{--}, \mathrm{HOO}^{\bullet}, \mathrm{H}_{2} \mathrm{O}_{2}$ ]. ROS may be also produced by ionising radiation.

Note 2: Singlet molecular dioxygen, $\mathrm{O}_{2}\left({ }^{1} \Delta_{\mathrm{g}}\right)$, one of the reactive oxygen species, is generally formed by energy transfer from an excited state to the ground state of dioxygen [7].

recombination (of free electrons and holes in solid photocatalyst)

Disappearance of free electrons and holes due to the transition of electrons from the conduction band to the valence band. Recombination can also occur by interaction of trapped or intra-bandgap electrons and holes or by the influence of impurities or defects in the crystalline structure.

Note 1: The recombination rate of photocarriers determines the stationary concentration of nonequilibrium carriers in a continuosly irradiated solid photocatalyst and thus determines the rates and quantum yields of a majority of heterogeneous photocatalytic reactions.

Note 2: The recombination of carriers obeys the energy and momentum conservation laws. Consequently, in wide bandgap energy solids, the radiative recombination is more favourable. In solids containing lattice imperfections (lattice defects) at moderate photoexcitation fluences, recombination through such defects (recombination centres) is more efficient than direct band-to-band recombination. 
recombination of photocarriers (via defects)

Two-step transition of an electron from the conduction band to a defect centre and then further to the valence band, or from the defect centre to the valence band (first step) and a subsequent transition of the conduction band electron to the defect centre (second step).

See also recombination centre.

\section{recombination centre}

Defect in semiconductors and insulators capable of sequential capture of free charge carriers of both signs with high efficiency.

Note: Provides a path for the recombination of free electrons and holes at high rates. Typically, in imperfect wide-bandgap energy solids, the rate of photoinduced carrier recombination through recombination centres at moderate photon irradiance is larger than the rate of band-to-band recombination.

\section{reflectance, $\rho$}

Fraction of light reflected by a system. In full generality it must be treated as a directional property that is a function of the reflected direction, the incident direction, and the incident wavelength. However, it is also commonly averaged over the reflected hemisphere to give the hemispherical spectral reflectivity.

Note: $\quad$ Mathematical expression for the hemispherical spectral reflectivity: $\rho=P_{\lambda}^{\text {refl }} / P_{\lambda}^{0}$, where superscript 0 indicates incident radiant power (prior to absorption and reflection) and superscript refl means reflected radiant power.

See $[8,9]$.

\section{regular surface}

Perfect surface of a solid without heterogeneity or defect.

Note: Rigorously speaking, this is a theoretical notion. In practice, however, this term is used to refer to local space regions of real relaxed and reconstructed surfaces if the perturbations caused by the nearest adjacent (surface) defects can be neglected.

See also surface.

\section{regular surface site}

Zero-dimension site (atom, ion, or interstitial position) at a regular surface.

Note: It can be a photocatalytic centre (site) in the ground (not active) state in some particular cases of heterogeneous photocatalysis as, e.g., in the case when pre-physisorbed entities trap photocarriers in the course of the photocatalysed reaction.

\section{relative photonic efficiency, $\xi_{\text {rel }}$}

Ratio of the photonic efficiency $(\xi)$ of a chemical process to the corresponding photonic efficiency of a standard process under otherwise identical conditions in the same reactor configuration and operation.

Note 1: For measurements carried out at constant photon flux, $\xi_{\text {rel }}$ can be estimated as the ratio of the corresponding reaction initial rates (for $t \rightarrow 0$ ), determined under identical conditions. 
Note 2: Protocols for the determination of $\xi_{\text {rel }}$ have been reported [4].

Note 3: See the restrictions about the determination of quantum yields in photocatalysis mentioned in the Introduction.

\section{ROS}

Acronym for reactive oxygen species.

\section{RTE}

Abbreviation of radiative transfer equation.

\section{sacrificial acceptor}

Molecular entity that acts as the electron acceptor in a photoinduced electron transfer process and is not restored in a subsequent oxidation process, but is destroyed by irreversible chemical conversion.

See [8].

\section{sacrificial donor}

Molecular entity that acts as the electron donor in a photoinduced electron transfer process and is not restored in a subsequent oxidation process, but is destroyed by irreversible chemical conversion.

See [8].

\section{scattering albedo}

Efficiency of scattering, i.e., ratio of scattering coefficient, $\sigma\left(\lambda_{\mathrm{i}}\right)$, to the total attenuance coefficient, $\beta\left(\lambda_{\mathrm{i}}\right)$, at a particular wavelength $\lambda_{\mathrm{i}}$.

Note 1: Mathematical expression: $\sigma\left(\lambda_{\mathrm{i}}\right) / \beta\left(\lambda_{\mathrm{i}}\right)$. Dimension one.

Note 2: A scattering albedo 1 indicates total scattering, whereas a scattering albedo 0 indicates that all attenuance is due to absorption.

\section{scattering coefficient (linear), $\sigma(\lambda)$}

Fraction of light scattered per unit distance in a participating medium.

Note 1: Component of attenuance due to scattering divided by the pathlength, $l$, within the scattering medium.

Note 2: The fraction due to absorbance at $\lambda_{\mathrm{i}}, A\left(\lambda_{\mathrm{i}}\right)$, should be subtracted from the total attenuance at the same wavelength, $D\left(\lambda_{\mathrm{i}}\right)$.

Note 3: Mathematical expression $\sigma\left(\lambda_{\mathrm{i}}\right)=\left[D_{\mathrm{e}}\left(\lambda_{\mathrm{i}}\right)-A_{\mathrm{e}}\left(\lambda_{\mathrm{i}}\right)\right] / l$. SI unit is $\mathrm{m}^{-1}$.

In practice, the spectral naperian scattering coefficient, $\sigma_{\lambda}$, is used. The subscript can be omitted but the wavelength should be specified.

See specific napierian (spectral) scattering coefficient. See [8].

\section{scattering in}

in-scattering 
scattering out

out-scattering

\section{semiconductor}

Material whose electric conductivity, $\kappa$, increases exponentially with increasing temperature owing to the thermal generation of free charge carriers, following van't Hoff's law:

$$
\kappa=\kappa_{0} \exp \left(-\Delta_{\mathrm{c}} H / \mathrm{RT}\right)
$$

where $\Delta_{c} H$ is the enthalpy of conduction.

Note 1: The bandgap energy of an intrinsic semiconductor can be as high as about $2 \mathrm{eV}$. An intrinsic semiconductor is a material with negligible concentration of defects and impurities and for which thermal excitation leads to band-to-band generation of both electrons and holes with identical concentrations of both types of carriers. This requires a small bandgap energy of the semiconductor.

Note 2: As crystals of these materials are grown, they are doped with traces of other elements to make regions that are $\mathrm{n}$ - or $\mathrm{p}$-type.

Note 3: An n-type semiconductor is a material in which electrons are the majority carriers owing to the presence of shallow donor intrinsic defects and/or impurities in the lattice. A p-type semiconductor is a material in which the holes are the majority carriers owing to the presence of shallow acceptor intrinsic defects and/or impurities in the lattice.

Note 4: The presence of defects and impurities (doping) in n- or p-type semiconductors leads to the observation of semiconductor behaviour in materials with a wider bandgap energy than in intrinsic semiconductors. For example, $\mathrm{ZnO}$ and $\mathrm{TiO}_{2}$, having a bandgap energy of about $3 \mathrm{eV}$, behave as n-type semiconductors.

Note 5: Where n- and p-type regions adjoin, a junction appears that will pass current in one direction (from $\mathrm{p}$ to $\mathrm{n}$ ) but not the other, giving a diode.

Compare with dielectrics, see $[8,21,22]$.

\section{sensitisation}

photosensitisation

radiation sensitisation

See [8].

\section{sensitised photoreactions}

See catalysed photochemical reaction, energy transfer, photocatalysis.

\section{sensitiser}

See [8].

\section{shallow (energy) trap}

Energy level (of trapped carrier) within the bandgap energy, near the bottom of the conduction band (electron shallow trap) and near the top of the valence band (hole shallow band) at an energy such that $E_{\text {trap }} \approx k T$, with $k$ the Boltzmann constant and $T$ the thermodynamic temperature. See [22]. 


\section{Solonitsyn effect}

post-adsorption

Adsorption (or reaction) after preliminary irradiation of a photocatalyst in vacuum (or in the absence of reagent).

Compare with Coekelbergs effect.

See memory effect $[27,44,47]$.

\section{sonophotocatalytic reactor}

Reactor in which a hybrid technique combining "sonochemical" and photocatalytic reactions is used.

Note: This type of reactor has been proposed for the treatment of wastewaters.

See [63] for a critical evaluation of this technology.

\section{space charge region}

Region formed within a semiconductor in contact with another phase in which there is a band bending that can be upward or downward (see depletion layer).

See [21].

\section{specific adsorption}

Refers to adsorption by short-range interactions (in addition to possible long-range coulombic interactions) between a species and the liquid/solid interphase in a semiconductor, allowing the penetration of the species into the inner layer and enabling its contact with the surface.

\section{specific photoadsorption number}

Maximum number of entities (molecules, atoms or radicals) adsorbed as a result of irradiation divided by the surface area and by the mass of adsorbent.

Note: This definition, per area and per mass, is misleading. It is included for historical reasons and was originally called specific photoadsorption capacity [64].

\section{specific (spectral) absorption coefficient (napierian $\alpha_{\lambda}^{*}$ or decadic $a_{\lambda}^{*}$ )}

Linear napierian, $\alpha_{\lambda}$, or linear decadic, $a_{\lambda}$, absorption coefficient divided by the mass concentration, $\rho$.

Note 1: The asterisk as superscript indicates that it is a specific property.

Note 2: Absorption coefficient is always a spectral property; the subscript $\lambda$ may be omitted but the wavelength should be specified.

Note 3: Mathematical definition:

$$
\alpha_{\lambda}^{*}=\frac{\alpha_{\lambda}}{\rho} \text { or } a_{\lambda}^{*}=\frac{a_{\lambda}}{\rho}
$$

SI unit is $\mathrm{m} \cdot \mathrm{kg}^{-1}$, common unit is $\mathrm{m} \cdot \mathrm{g}^{-1}$ or $\mathrm{cm}^{2} \cdot \mathrm{g}^{-1} \cdot \mathrm{nm}^{-1}$.

Note 4: For reacting systems with mass fluctuations and high scattering, space- and timedependent specific, napierian and decadic, absorption coefficients are necessary, i.e., $\alpha_{\lambda}^{*}(\boldsymbol{x}, t)$ and $a_{\lambda}^{*}(\boldsymbol{x}, t)$, respectively; $\boldsymbol{x}$ defines the position in a three-dimensional space.

See [8]. 
specific (spectral) napierian attenuance coefficient, $\beta_{\lambda}^{*}$

specific napierian (spectral) extinction coefficient

Attenuance (napierian), $D_{\mathrm{e}}$, divided by the absorption pathlength, $l$, and the mass concentration, $\rho$.

Note 1: Mathematical expression:

$$
\beta_{\lambda}^{*}=\left(\frac{1}{\rho l}\right) \ln \left(\frac{P_{\lambda}^{0}}{P_{\lambda}}\right)=\frac{D_{\mathrm{e}, \lambda}}{\rho l}
$$

where $P_{\lambda}^{0}$ and $P_{\lambda}$ are, respectively, the incident and transmitted spectral radiant power or the incident and transmitted spectral photon flux.

Note 2: In common use for $l$ in $\mathrm{cm}$ and $\rho$ in $\left(\mathrm{g} \cdot \mathrm{cm}^{-3}\right), \beta_{\lambda}^{*}$ is in $\mathrm{cm}^{2} \cdot \mathrm{g}^{-1}$.

Note 3: Sometimes called mass napierian (spectral) attenuance coefficient.

Note 4: Strictly speaking, the spectral quantity should be used, which is the derivative of the specific napierian attenuance coefficient with respect to $\lambda$, i.e., $\mathrm{d} \beta *(\lambda) / \mathrm{d} \lambda$ in common units $\mathrm{cm}^{2} \cdot \mathrm{g}^{-1} \cdot \mathrm{nm}^{-1}$ or in $\mathrm{cm} \cdot \mathrm{g}^{-1}$. In practice, the subscript is omitted and the respective unit is not taken into account. The wavelength should be specified.

Note 5: The specific napierian attenuance coefficient reduces to specific napierian absorption coefficient for a non-reflecting and non-scattering sample.

See absorbance, absorption coefficient, Beer-Lambert law in [7].

specific (spectral) napierian scattering coefficient, $\sigma_{\lambda}^{*}$

Fraction of attenuance (napierian) not due to absorbance, divided by the pathlength, $l$, within the scattering medium and the mass concentration, $\rho$.

Note 1: Mathematical expression: $\sigma_{\lambda}^{*}=\sigma_{\lambda} / \rho=\left[D_{\mathrm{e}, \lambda}-A_{\mathrm{e}, \lambda}\right] /(l \rho)$, where $\rho$ is the mass concentration. The absorbance should be subtracted from the total attenuance for the calculation of $\sigma_{\lambda}$ and $\sigma_{\lambda}^{*}$. Common unit is $\mathrm{cm}^{2} \cdot \mathrm{g}^{-1}$.

Note 2: The superscript asterisk indicates that it is a specific property.

Note 3: Sometimes called mass napierian (spectral) scattering coefficient.

Note 4: Strictly speaking, the spectral quantity should be used, which is the derivative of the specific napierian scattering coefficient with respect to $\lambda$, i.e., $\mathrm{d} \sigma *(\lambda) / \mathrm{d} \lambda$ in common units $\mathrm{cm}^{2} \cdot \mathrm{g}^{-1} \cdot \mathrm{nm}^{-1}$ or in $\mathrm{cm} \cdot \mathrm{g}^{-1}$. In practice, the subscript is omitted and the respective unit is not taken into account. The wavelength should be specified.

See [8].

specific surface area, $a^{s}$

Surface area divided by the mass of the relevant phase. Common unit is $\mathrm{m}^{2} \cdot \mathrm{g}^{-1}$.

See [8].

spectral fluence rate

See fluence rate [8]. 
spectral irradiance, $E_{\lambda}$

Derivative of irradiance, $E$, with respect to wavelength, $\lambda$. SI unit is $\mathrm{W} \cdot \mathrm{m}^{-3}$, common unit is $\mathrm{W} \cdot \mathrm{m}^{-2} \cdot \mathrm{nm}^{-1}$.

Note: All spectral terms may also be defined as derivatives with respect to frequency, $v$, or wavenumber, $\widetilde{v}$ and are referred to, when necessary, as in terms of wavelength, or frequency or wavenumber, respectively.

See [8].

spectral photon flux, $q_{\mathrm{p}, \lambda}, \Phi_{\mathrm{p}, \lambda}$

Derivative of photon flux, number basis, $q_{\mathrm{p}}$, with respect to wavelength, $\lambda$.

SI unit is $\mathrm{s}^{-1} \cdot \mathrm{m}^{-1}$; Coherent SI unit is $\mathrm{s}^{-1} \cdot \mathrm{nm}^{-1}$.

Note 1: This quantity can be expressed on a chemical amount basis by dividing $q_{\mathrm{p}, \lambda}$ by the Avogadro constant, the name then being "spectral photon flux, amount basis", the symbol $q_{n, \mathrm{p}, \lambda}$ and the SI unit $\mathrm{mol} \cdot \mathrm{s}^{-1} \cdot \mathrm{m}^{-1}$; common unit is einstein $\cdot \mathrm{s}^{-1} \cdot \mathrm{nm}^{-1}$.

Note 2: $q_{\mathrm{p}, \lambda}$ is preferred, because in photochemistry $\Phi$ is reserved for quantum yield.

Note 3: All spectral terms may also be defined as derivatives with respect to frequency, $v$, or wavenumber, $\widetilde{v}$ and are referred to, when necessary, as in terms of wavelength, or frequency or wavenumber, respectively.

See [8].

spectral photon irradiance, $E_{\mathrm{p}, \lambda}$

Derivative of photon irradiance, $E_{\mathrm{p}}$, with respect to wavelength, $\lambda$.

Note 1: Mathematical expression: $E_{\mathrm{p}, \lambda}=\mathrm{d} E_{\mathrm{p}} / \mathrm{d} \lambda$.

SI unit is $\mathrm{s}^{-1} \cdot \mathrm{m}^{-3}$; common unit is $\mathrm{s}^{-1} \cdot \mathrm{m}^{-2} \cdot \mathrm{nm}^{-1}$.

Note 2: This quantity can be expressed on a chemical amount basis by dividing $E_{\mathrm{p}, \lambda}$ by the Avogadro constant, the name then being "spectral photon irradiance, amount basis", the symbol $E_{n, \mathrm{p}, \lambda}$ and the SI unit $\mathrm{mol} \cdot \mathrm{s}^{-1} \cdot \mathrm{m}^{-3} ;$ common unit is einstein $\cdot \mathrm{s}^{-1} \cdot \mathrm{m}^{-2} \cdot \mathrm{nm}^{-1}$.

Note 3: All spectral terms may also be defined as derivatives with respect to frequency, $v$, or wavenumber, $\widetilde{v}$ and are referred to, when necessary, as in terms of wavelength, or frequency or wavenumber, respectively.

See $[8]$.

spectral photon radiance, $L_{\mathrm{p}, \Omega, \lambda}$

Derivative of photon radiance for a divergent beam, $L_{\mathrm{p}, \Omega}$, with respect to wavelength, $\lambda$.

SI unit is $\mathrm{s}^{-1} \cdot \mathrm{m}^{-3} \cdot \mathrm{sr}^{-1}$; common unit is $\mathrm{s}^{-1} \cdot \mathrm{m}^{-2} \cdot \mathrm{sr}^{-1} \cdot \mathrm{nm}^{-1}$.

Note 1: Mathematical expression for a divergent beam leaving or passing through a small transparent element of surface in a given direction from the source about the solid angle $\Omega$, divided by the solid angle and by the orthogonally projected area of the element in a plane normal to the given beam direction, $\mathrm{d} S_{\perp}=\mathrm{d} S \cos \theta$, with $\theta$ the angle between the normal to the surface and the direction of the beam.

$$
L_{\mathrm{p}, \Omega, \lambda}=\lim _{(\Delta \Omega, \Delta S, \Delta t, \Delta \lambda) \rightarrow 0} \frac{\Delta N_{\mathrm{p}}}{\Delta \Omega \Delta S \cos \theta \Delta t \Delta \lambda}
$$


Note 2: Spectral photon radiance of a divergent beam is the quantity used when working in three-dimensional reaction spaces, such as large photochemical reactors and the corresponding up-scaling calculations. The mathematical expression above takes into account scattering and has a local value at any time for any point inside the reaction space.

Note 3: This quantity can be expressed on a chemical amount basis by dividing $L_{\Omega, \mathrm{p}, \lambda}$ by the Avogadro constant, the name then is "spectral photon radiance, chemical amount basis".

$\mathrm{SI}$ unit is $\mathrm{mol} \cdot \mathrm{s}^{-1} \cdot \mathrm{m}^{-3} \cdot \mathrm{sr}^{-1}$; common unit is einstein $\cdot \mathrm{s}^{-1} \cdot \mathrm{m}^{-2} \cdot \mathrm{sr}^{-1} \cdot \mathrm{nm}^{-1}$.

Note 4: All spectral terms may also be defined as derivatives with respect to frequency, $v$, or wavenumber, $\widetilde{v}$, and are referred to, when necessary, as in terms of wavelength, frequency, or wavenumber, respectively.

See [8].

\section{spectral photonic yield}

Dependence of the photonic yield on the photon energy (wavelength, frequency, or wavenumber) of the radiation for a given photoreaction obtained at a certain incident (prior to absorption) photon irradiance in a given spectral range.

Note 1: In practice, it is normalised to a certain spectral photon flux provided that photoresponse scales linearly with it.

Note 2: Information concerning the photoexcitation of a solid photocatalyst can be obtained from a comparison of the spectral quantum yield with the spectral photonic efficiency.

\section{spectral quantum yield}

Dependence of the quantum yield on the photon energy (wavelength, frequency or wavenumber) of the radiation absorbed by the reactant for a given photoreaction.

Note: To obtain information on the spectral variation of activity of a photocatalyst, the measured photoresponse must scale linearly with the photon flux at all wavelengths.

spectral radiance, $L_{\lambda}$

Derivative of radiance, $L$, with respect to wavelength, $\lambda$.

Note 1: Mathematical expression:

$$
L_{\Omega, \lambda}=\lim _{(\Delta \Omega, \Delta S, \Delta \lambda, \Delta t) \rightarrow 0} \frac{\Delta P}{\Delta \Omega \Delta S \cos \theta \Delta \lambda}
$$

For a divergent beam, SI unit is $\mathrm{W} \cdot \mathrm{m}^{-3} \cdot \mathrm{sr}^{-1}$; common unit is $\mathrm{W} \cdot \mathrm{m}^{-2} \cdot \mathrm{sr}^{-1} \cdot \mathrm{nm}^{-1}$. For a parallel beam, SI unit is $\mathrm{W} \cdot \mathrm{m}^{-3}$; common unit is $\mathrm{W} \cdot \mathrm{m}^{-2} \cdot \mathrm{nm}^{-1}$.

Note 2: All spectral terms may also be defined as derivatives with respect to frequency, $v$, or wavenumber, $\widetilde{v}$, and are referred to, when necessary, as in terms of wavelength, frequency, or wavenumber, respectively.

See spectral photon radiance.

See [8]. 
spectral radiant power, $P_{\lambda}$

Derivative of radiant power, $P$, with respect to wavelength $\lambda$. SI unit is $\mathrm{W} \cdot \mathrm{m}^{-1}$; common unit is $\mathrm{W} \cdot \mathrm{nm}^{-1}$.

Note: All spectral terms may also be defined as derivatives with respect to frequency, $v$, or wavenumber, $\widetilde{v}$ and are referred to, when necessary, as in terms of wavelength, or frequency or wavenumber, respectively.

See [8].

Stern layer

Helmholtz layer

inner layer

compact layer

Counter and co-ions in immediate contact with a surface are said to be located in the Stern layer.

Note: It may have a solvent structure that differs from that of the bulk solution. Solvent differences may occur due to interactions with the surface and strong solvation (e.g., hydration) of the ions in the Stern layer.

See $[8,30]$.

\section{Stern plane}

outer Helmholtz plane

\section{surface}

External monolayer of a solid particle consisting of the regular array of surface atoms (or ions) and intrinsic and extrinsic surface defects of various types.

Note: Surface properties are denoted by a superscript s, such as amount of adsorbed substance, e.g., surface concentration of photocarriers $n^{\mathrm{s}}[9]$.

See [8].

\section{surface-active centre}

surface-active site

\section{surface-active site}

surface-active centre

Surface regular or defect site that can initiate the chemical transformation of reagents.

Note: See [65] for an updated discussion of the role of surface-active centres in photocatalytic reactions.

See $F$ centres, $V$ centres.

surface concentration of photocarriers, $n^{\mathrm{s}}$

Number of photocarriers per volume in the spatial region of the surface of an irradiated solid (photocatalyst) particle. SI unit is $\mathrm{m}^{-3}$ or $\mathrm{mol} \cdot \mathrm{m}^{-3}$. 
Note 1: $n^{\mathrm{s}}$ is determined by the rate of carrier generation within the depth of radiation penetration, by the lifetimes of free photocarriers, by the rate of surface recombination, by the diffusion length of carriers, by the electric field in the subsurface area, and by the characteristic particle size.

Note 2: $n^{\mathrm{s}}$ is often used in theoretical models of photocatalytic reactions.

\section{surface coverage, $\theta$}

Number of adsorbed entities (molecules, ions or radicals) on a surface divided by the number of entities in a filled monolayer on that surface [8].

Note: Dimension one (it is a fractional number).

See adsorption.

surface $\mathbf{F}$ centres

See colour centres [23,29].

surface recombination

Recombination of charge carriers at a solid surface or at an interface between different phases.

Note: In theoretical models of heterogeneous photocatalysis, the photocarriers exchange between the solid and the reactants yielding products that may be considered as a specific type of surface recombination products.

See rate of surface recombination.

\section{surface states}

Energy levels localised in the surface region of semiconductors, which do not bear any direct relation to the bulk energy distribution, but which can exchange electrons with the bulk. The energy levels are located particularly within the energy gap of semiconductors and insulators and originate from a regular surface and from any surface defects, including adsorbed species.

See [8].

\section{surface $\mathbf{V}$ centres}

See colour centres [35].

\section{thermal bleaching}

Loss of absorption by the colour centres because of heating of the solid semiconductor or insulator.

\section{thermophotocatalysis (light-initiated thermocatalytic phenomenon)}

A photocatalytic phenomenon occurring when the system is also heated by the ultraviolet, visible, or infrared radiation.

Note: In case of high photon flux, thermochemical processes may take place as a result of radiationless deactivation of excited species, in addition to photochemical processes. This often occurs upon exposure of a heterogeneous photocatalyst to pulsed IR-light or pulsed laser emission.

See photocatalysis. 


\section{TOF}

Acronym for turnover frequency.

Same acronym used for time-of-flight, in particular in connection with mass spectrometry.

\section{TON}

Acronym for turnover number.

\section{transmittance}

See attenuance.

\section{turnover frequency, $\left(\tau_{\mathrm{F}}\right.$, TOF $)$}

turnover rate

Number of photoinduced transformations (product formed or reactant consumed), per catalytic site and per time period.

Note 1: Mathematical expression:

$$
\tau_{\mathrm{F}}=\frac{1}{N_{\mathrm{a}}} \frac{\mathrm{d} N}{\mathrm{~d} t}
$$

where $N_{\mathrm{a}}$ is the number of the catalyst active sites, $N$ is the number of photocatalytic events. This definition of TOF should be used whenever possible. SI unit is $\mathrm{s}^{-1}$.

Note 2: In heterogeneous photocatalysis, when the number of active sites is not known, the surface area should be used to normalise the number of turnovers and a different quantity results, i.e., the areal turnover frequency, $\tau_{\text {het }}$

$$
\tau_{\text {het }}=\frac{1}{S} \frac{\mathrm{d} N}{\mathrm{~d} t}
$$

where $S$ is the $\operatorname{BET}\left(\mathrm{N}_{2}\right)$ area of the photocatalyst. SI unit is $\mathrm{m}^{-2} \cdot \mathrm{s}^{-1}$.

Note 3: In homogeneous photocatalysis, the $\tau_{\text {hom }}$ value is used per photocatalyst entity. In this case, the turnover frequency can be expressed also as the number of photocatalytic cycles per number concentration of the photocatalyst, $C_{\mathrm{m}}$. SI unit is s-1.

$$
\tau_{\text {hom }}=\frac{1}{C_{\mathrm{m}}} \frac{\mathrm{d} N}{\mathrm{~d} t}
$$

See also turnover number.

Note 4: Same acronym is used for time-of-flight, in particular in connection with mass spectrometry.

See $[13,66]$.

\section{turnover number (TON)}

Number of times, $n$, that the overall reaction (the photochemical transformation) goes through a photocatalytic cycle.

Note 1: It can be equally applied to homogeneous and heterogeneous systems. 
Note 2: TON is a number, whereas in traditional catalysis, e.g., enzyme kinetics, it is defined per certain time period.

Note 3: In heterogeneous photocatalysis it is the ratio of the number of photoinduced transformations for a given period of time to the number of active sites or photocatalytic centres in their ground state. Should the number of active sites be known, TON could be expressed per number of sites.

Note 4: TON is a quantity of dimension one attributed in photocatalysis to the overall system that contains the photocatalyst, even if, in heterogeneous photocatalysis, part of the photocatalyst active centres appears to be not irradiated.

Note 5: In homogeneous photocatalysis it is the ratio of the number of photoinduced transformations for a given period to the number of ground state photocatalyst entities.

\section{turnover rate (TOR)}

turnover frequency

\section{upward band bending}

See depletion layer.

\section{UV dose}

Dose of UV radiation.

Note: This term is also used widely in UV disinfection applications having the same meaning as fluence; this use is discouraged.

See [8].

\section{V centres}

Trapped holes in semiconductors.

See colour centres [35].

\section{valence band}

Highest energy continuum of energy levels in a solid that is fully occupied by electrons at $0 \mathrm{~K}$.

Note 1: The valence band is lower in energy than the conduction band and is generally completely full in semiconductors. When heated, electrons from the valence band jump out of the band across the band gap and into the conduction band, making the material conductive. The Fermi level at energy $E_{\mathrm{F}}$ separates the valence band from the conduction band.

Note 2: In metals, the valence band is the conduction band.

See bandgap energy, conduction band, Fermi level [8,22].

\section{VOC}

Acronym for volatile organic compound. 
volatile organic compounds (VOCs)

Term commonly used to designate organic gas-phase pollutants frequently degraded by photocatalytic procedures.

Note: Often used for any organic compound with a vapor pressure higher than $10 \mathrm{~Pa}$ at $T=$ $293 \mathrm{~K}$.

See [68].

\section{QUANTITIES, SYMBOLS, AND TERMS USED IN THIS DOCUMENT ${ }^{1}$}

\begin{tabular}{|c|c|c|c|c|}
\hline Name & Symbol & Definition & SI unit & Notes \\
\hline $\begin{array}{l}\text { absorbance } \\
\text { decadic } \\
\text { napierian }\end{array}$ & $\begin{array}{l}A, A_{10} \\
A_{\mathrm{e}}\end{array}$ & $\begin{array}{l}A=\lg \left(P_{\lambda}^{0} / P_{\lambda}\right)=-\lg T \\
A_{\mathrm{e}}=\ln \left(P_{\lambda}^{0} / P_{\lambda}\right)=-\ln T \\
\text { with } P_{\lambda}^{0} \text { and } P_{\lambda} \text { the spectral radiant } \\
\text { power incident (superscript } 0, \text { zero) } \\
\text { and transmitted (no superscript), } \\
\text { respectively. }\end{array}$ & 1 & 2 \\
\hline $\begin{array}{l}\text { absorbed local } \\
\text { spectral photon } \\
\text { flux density, } \\
\text { local volumetric } \\
\text { rate of photon } \\
\text { absorption, LVRPA }\end{array}$ & $L_{\mathrm{p}, \lambda}^{\mathrm{a}}(\boldsymbol{x}, t)$ & $\begin{array}{l}L_{\mathrm{p}, \lambda}^{\mathrm{a}}(\boldsymbol{x}, t)=\alpha_{\lambda}(\boldsymbol{x}, t) E_{\mathrm{p}, \lambda, \mathrm{o}}(\boldsymbol{x}, t) \\
\text { with } E_{\mathrm{p}, \lambda, \mathrm{o}}(\boldsymbol{x}, t)=\int_{4 \pi} L_{\mathrm{p}, \lambda}(\boldsymbol{x}, t) \mathrm{d} \Omega \\
\text { the spectral photon fluence rate incident } \\
\text { from all directions at } \boldsymbol{x}, \text { and } \alpha_{\lambda}(\boldsymbol{x}, t) \\
\text { the space- and time-dependent linear } \\
\text { napierian absorption coefficient. }\end{array}$ & $\begin{array}{l}\mathrm{s}^{-1} \cdot \mathrm{m}^{-4} \\
\text { or } \\
\mathrm{mol} \cdot \mathrm{s}^{-1} \cdot \mathrm{m}^{-4}\end{array}$ & 3 \\
\hline $\begin{array}{l}\text { absorbed local } \\
\text { spectral radiance, } \\
\text { local volumetric } \\
\text { rate of energy } \\
\text { absorption, LVREA }\end{array}$ & $L_{\lambda}^{\mathrm{a}}(\boldsymbol{x}, t)$ & $\begin{array}{l}L_{\lambda}^{\mathrm{a}}(\boldsymbol{x}, t)=\alpha_{\lambda}(\boldsymbol{x}, t) E_{\lambda, \mathrm{o}}(\boldsymbol{x}, t) \\
E_{\lambda, \mathrm{o}}(\boldsymbol{x}, t) \text { is the space- and time-dependent } \\
\text { spectral fluence rate incident from all } \\
\text { directions at } \boldsymbol{x}, \alpha_{\lambda}(\boldsymbol{x}, t) \text { is the } \\
\text { space- and time-dependent linear } \\
\text { napierian absorption coefficient. }\end{array}$ & $\mathrm{W} \cdot \mathrm{m}^{-4}$ & 4 \\
\hline $\begin{array}{l}\text { absorbed (spectral) } \\
\text { photon flux } \\
\text { density, absorbed } \\
\text { (spectral) photon } \\
\text { radiance }\end{array}$ & $\left\langle L_{\mathrm{p}, \lambda}^{\mathrm{a}}(t)\right\rangle_{V}$ & $\begin{array}{l}\left\langle L_{\mathrm{p}, \lambda}^{\mathrm{a}}(t)\right\rangle_{V}=\frac{1}{V} \int_{V} L_{\mathrm{p}, \lambda}^{\mathrm{a}}(\boldsymbol{x}, t) \mathrm{d} V= \\
\frac{1}{V} \int_{V} \alpha_{\lambda}(\boldsymbol{x}, t) E_{\mathrm{p}, \lambda, \mathrm{o}}(\boldsymbol{x}, t) \mathrm{d} V \\
E_{\mathrm{p}, \lambda, \mathrm{o}}^{\prime}(\boldsymbol{x}, t) \text { is the space- and } \\
\text { time-dependent spectral photon fluence } \\
\text { rate incident from all directions at } \\
\boldsymbol{x}, \alpha_{\lambda}(\boldsymbol{x}, t) \text { is the space- and } \\
\text { time-dependent linear napierian } \\
\text { absorption coefficient. }\end{array}$ & $\begin{array}{l}\mathrm{s}^{-1} \cdot \mathrm{m}^{-4} \\
\text { or } \\
\mathrm{mol} \cdot \mathrm{s}^{-1} \cdot \mathrm{m}^{-4}\end{array}$ & 5 \\
\hline
\end{tabular}


(Continued)

\begin{tabular}{|c|c|c|c|c|}
\hline Name & Symbol & Definition & SI unit & Notes \\
\hline $\begin{array}{l}\text { absorbed } \\
\text { (spectral) radiant } \\
\text { density }\end{array}$ & $\left\langle L_{\lambda}^{\mathrm{a}}(t)\right\rangle_{V}$ & $\begin{array}{l}\left\langle L_{\lambda}^{\mathrm{a}}(t)\right\rangle_{V}=\frac{1}{V} \int_{V} L_{\lambda}^{\mathrm{a}}(\boldsymbol{x}, t) \mathrm{d} V= \\
\frac{1}{V} \int_{V} \alpha_{\lambda}(\boldsymbol{x}, t) E_{\lambda, \mathrm{o}}(\boldsymbol{x}, t) \mathrm{d} V \\
E_{\lambda, \mathrm{o}}(\boldsymbol{x}, t) \text { is the space- and time-dependent } \\
\text { spectral fluence rate incident from } \\
\text { all directions at } \boldsymbol{x}, \alpha_{\lambda}(\boldsymbol{x}, t) \text { is the } \\
\text { space- and time-dependent linear } \\
\text { napierian absorption coefficient. }\end{array}$ & $\mathrm{W} \cdot \mathrm{m}^{-4}$ & 6 \\
\hline
\end{tabular}

absorption

(term in the RTE)
$\alpha_{\lambda}(\boldsymbol{x}, t) L_{\Omega, \lambda}(\boldsymbol{x}, t)$
with $\alpha_{\lambda}(\boldsymbol{x}, t)$ the space- and
time-dependent spectral linear
napierian absorption coefficient
and $L_{\Omega, \lambda}(\boldsymbol{x}, t)$ the incident spectral
radiance.

$\mathrm{W} \cdot \mathrm{sr}^{-1} \cdot \mathrm{m}^{-4} \quad 7$

\begin{abstract}
absorption coefficient
linear decadic

molar decadic

linear napierian

molar napierian

specific decadic

specific napierian
absorption
cross-section

$\sigma(\lambda)$

$a$

$\varepsilon$

$\alpha$

$\kappa$

$a *$

$\alpha^{*}$

amount of photons

amount

concentration of B

area

attenuance

Avogadro constant

bandgap energy

$$
n_{\mathrm{p}}
$$

$S$

$D$
$c,[\mathrm{~B}]$

$$
\begin{aligned}
& D=\lg \left(P_{\lambda}^{0} / P_{\lambda}\right)=-\lg T(\lambda) \\
& D_{\mathrm{e}}=\ln \left(P_{\lambda}^{0} / P_{\lambda}\right)=-\ln T(\lambda) \\
& \text { with } P_{\lambda}^{0} \text { and } P_{\lambda} \text { the spectral radiant } \\
& \text { power incident (superscript 0, zero) } \\
& \text { transmitted (no superscript), } \\
& \text { respectively. }
\end{aligned}
$$$$
\text { power incident (superscript } 0 \text {, zero) and }
$$

$$
\begin{aligned}
& a=A_{10} / l \\
& \varepsilon=a / c=A_{10} / c l \\
& \alpha=A_{\mathrm{e}} / l \\
& \kappa=\alpha / c=A_{\mathrm{e}} / c l \\
& a^{*}=a / \rho
\end{aligned}
$$$$
\sigma(\lambda)=\kappa(\lambda) / N_{\mathrm{A}}=\ln 10 \varepsilon(\lambda) / N_{\mathrm{A}}
$$$$
\text { with } \kappa(\lambda) \text { the molar napierian absorption }
$$$$
\text { coefficient. }
$$

$\begin{array}{lc}\mathrm{m}^{-1} & 8 \\ \mathrm{~m}^{2} \cdot \mathrm{mol}^{-1} & 9 \\ \mathrm{~m}^{-1} & 8 \\ \mathrm{~m}^{2} \cdot \mathrm{mol}^{-1} & 9 \\ \mathrm{~m}^{2} \cdot \mathrm{kg}^{-1} & 10 \\ \mathrm{~m}^{2} \cdot \mathrm{kg}^{-1} & 10\end{array}$

$\mathrm{m}^{2}$

(1)


(Continued)

\begin{tabular}{|c|c|c|c|c|}
\hline Name & Symbol & Definition & SI unit & Notes \\
\hline $\begin{array}{l}\text { Brunauer-Emmett- } \\
\text { Teller surface area }\end{array}$ & BET & & $\mathrm{m}^{2} \cdot \mathrm{kg}^{-1}$ & 17 \\
\hline $\begin{array}{l}\text { Brunauer-Emmett- } \\
\text { Teller surface area, } \\
\text { using } \mathrm{N}_{2} \text { for the } \\
\text { measurement }\end{array}$ & $\operatorname{BET}\left(\mathrm{N}_{2}\right)$ & & $\mathrm{m}^{2} \cdot \mathrm{kg}^{-1}$ & 17 \\
\hline $\begin{array}{l}\text { conversion } \\
\text { cross-section }\end{array}$ & & $\begin{array}{l}\sigma \Phi \\
\text { with } \sigma \text { the absorption cross-section and } \\
\Phi \text { the reaction quantum yield. }\end{array}$ & $\mathrm{m}^{2}$ & 18 \\
\hline $\begin{array}{l}\text { differential } \\
\text { photodesorption } \\
\text { quantum yield }\end{array}$ & $\Phi_{\mathrm{des}}$ & $\begin{array}{l}\Phi_{\mathrm{des}}=\frac{\mathrm{d} N_{\mathrm{des}} / \mathrm{d} t}{q_{\mathrm{p}, \lambda}} \\
\text { where } \mathrm{d} N_{\mathrm{des}} / \mathrm{d} t \text { is the photodesorption } \\
\text { rate and } q_{\mathrm{p}, \lambda} \text { is the absorbed spectral } \\
\text { photon flux. }\end{array}$ & 1 & \\
\hline $\begin{array}{l}\text { differential } \\
\text { quantum yield } \\
\text { for an homogeneous } \\
\text { system }\end{array}$ & $\Phi(\lambda)$ & $\begin{array}{l}\Phi(\lambda)=\frac{\mathrm{d} x / \mathrm{d} t}{q_{n, \mathrm{p}, \lambda}^{0}\left[1-10^{-A}\right]} \\
\text { where } \mathrm{d} x / \mathrm{d} t \text { is the rate of change of a } \\
\text { quantity } x \text { measuring the reaction and } \\
q_{n, \mathrm{p}, \lambda}^{0} \text { is the incident spectral photon } \\
\text { flux, amount basis. }\end{array}$ & 1 & \\
\hline $\begin{array}{l}\text { differential } \\
\text { quantum yield } \\
\text { with heterogeneous } \\
\text { light distribution }\end{array}$ & $\Phi(\lambda)$ & $\begin{array}{l}\text { For a divergent beam } \\
\Phi(\lambda)=\frac{\mathrm{d} x / \mathrm{d} t}{\left\langle L_{\mathrm{p}, \lambda}^{\mathrm{a}}(t)\right\rangle_{V}} \\
\text { where } x \text { is the number concentration of } \\
\text { a product formed or reactant depleted } \\
\text { and }\left\langle L_{\mathrm{p}, \lambda}^{\mathrm{a}}(t)\right\rangle_{V} \text { is the volume averaged } \\
\text { absorbed (spectral) photon flux density. }\end{array}$ & 1 & \\
\hline einstein & $\mathrm{E}$ & one mole of photons & $\mathrm{mol}^{-1}$ & 12 \\
\hline $\begin{array}{l}\text { efficiency (of a } \\
\text { reaction step) }\end{array}$ & $\eta$ & $\begin{array}{l}\eta=k_{\mathrm{i}} /\left(\Sigma_{\mathrm{i}} k_{\mathrm{i}}\right) \text { with } k_{\mathrm{i}} \text { the first-order rate } \\
\text { constants for all decay processes of the } \\
\text { decaying state. }\end{array}$ & 1 & \\
\hline electric conductivity & $\kappa$ & & $\mathrm{S} \cdot \mathrm{m}^{-1}$ & 19 \\
\hline
\end{tabular}


(Continued)

\begin{tabular}{|c|c|c|c|c|}
\hline Name & Symbol & Definition & SI unit & Notes \\
\hline extinction coefficient & $\beta(\lambda)$ & $\begin{array}{l}\beta(\lambda)=\alpha(\lambda)+\sigma(\lambda)=D_{\mathrm{e}}(\lambda) / l \\
\text { with } \alpha(\lambda) \text { the linear napierian absorption } \\
\text { coefficient and } \sigma(\lambda) \text { the linear napierian } \\
\text { scattering coefficient. }\end{array}$ & $\mathrm{m}^{-1}$ & 20 \\
\hline $\begin{array}{l}\text { Fermi level } \\
\text { energy }\end{array}$ & $E_{\mathrm{F}}$ & & $\mathrm{J} \cdot \mathrm{mol}^{-1}$ & 16 \\
\hline $\begin{array}{l}\text { fluence, } \\
\text { radiant energy } \\
\text { fluence }\end{array}$ & $F_{\mathrm{o}}, H_{\mathrm{o}}$ & $F_{\mathrm{o}}=H_{\mathrm{o}}=\int_{t} E_{\mathrm{o}} \mathrm{d} t=\mathrm{d} Q / \mathrm{d} S$ & $\mathrm{~J} \cdot \mathrm{m}^{-2}$ & 21,22 \\
\hline $\begin{array}{l}\text { fluence rate } \\
\text { (see irradiance) }\end{array}$ & $E_{\mathrm{o}}$ & $\begin{array}{l}E_{\mathrm{o}}=\mathrm{d} F_{\mathrm{o}} / \mathrm{d} t \\
E_{\lambda, \mathrm{o}}(\boldsymbol{x}, t)=\int_{\Omega} L_{\lambda}(\boldsymbol{x}, t, \Omega) \mathrm{d} \Omega\end{array}$ & $\mathrm{W} \cdot \mathrm{m}^{-2}$ & 22,23 \\
\hline $\begin{array}{l}\text { fraction of light } \\
\text { absorbed or } \\
\text { scattered }\end{array}$ & $f(\lambda)$ & $f(\lambda)=1-T(\lambda)$ & 1 & \\
\hline frequency (linear) & $v$ & $\begin{array}{l}v=c / \lambda \\
\text { with } c \text { the velocity of light and } \lambda \text { the } \\
\text { wavelength. }\end{array}$ & $\mathrm{Hz}$ & \\
\hline frequency (angular) & $\omega$ & $\omega=2 \pi v$ & $\mathrm{rad} \cdot \mathrm{s}^{-1}$ & \\
\hline \multicolumn{5}{|l|}{$\begin{array}{l}G \text { value } \\
\text { see radiation- } \\
\text { chemical yield }\end{array}$} \\
\hline $\begin{array}{l}\text { half-life of a } \\
\text { transient species }\end{array}$ & $\tau_{1 / 2}, t_{1 / 2}$ & $c\left(t=\tau_{1 / 2}\right)=c(t=0) / 2$ & $\mathrm{~s}$ & \\
\hline $\begin{array}{l}\text { initial rate of } \\
\text { photoadsorption }\end{array}$ & & $\begin{array}{l}\mathrm{d} n / \mathrm{d} t \\
\text { in heterogeneous systems } \\
\mathrm{d} p / \mathrm{d} t \text { in a gas/solid system } \\
\mathrm{d} c / \mathrm{d} t \text { in a liquid/solid system } \\
\text { In every case for } t \rightarrow 0 \text {. }\end{array}$ & $\begin{array}{l}\mathrm{mol} \cdot \mathrm{s}^{-1} \\
\mathrm{~Pa} \cdot \mathrm{s}^{-1} \\
\mathrm{~mol} \cdot \mathrm{L}^{-1} \cdot \mathrm{s}^{-1}\end{array}$ & 24 \\
\hline in-scattering & $\mathrm{Sc}_{\text {in }}$ & $\begin{array}{l}\mathrm{Sc}_{\text {in }}= \\
\frac{\sigma_{\lambda}(x, t)}{4 \pi} \int_{4 \pi} B_{\lambda}\left(\Omega^{\prime} \rightarrow \Omega\right) L_{\Omega, \lambda}(x, t) \mathrm{d} \Omega^{\prime} \\
B_{\lambda} \text { is the phase function for scattering } \\
\text { distribution and } L_{\Omega, \lambda} \text { is the incident } \\
\text { spectral radiance. }\end{array}$ & $\mathrm{W} \cdot \mathrm{sr}^{-1} \cdot \mathrm{m}^{-4}$ & 25 \\
\hline
\end{tabular}


(Continued)

\begin{tabular}{|c|c|c|c|c|}
\hline Name & Symbol & Definition & SI unit & Notes \\
\hline $\begin{array}{l}\text { irradiance } \\
\text { (radiant power } \\
\text { received) }\end{array}$ & E & $E=\int_{2 \pi} L_{\mathrm{e}} \cos \theta \mathrm{d} \Omega=\mathrm{d} P / \mathrm{d} S$ & $\mathrm{~W} \cdot \mathrm{m}^{-2}$ & 26 \\
\hline lifetime & $\tau$ & $\begin{array}{l}\tau=1 / k=1 /\left(\Sigma_{l} k_{i}\right) \text { with } k_{i} \text { the first-order } \\
\text { rate constants for all decay processes of } \\
\text { the decaying state. } \\
c(t=\tau)=c(t=0) / \mathrm{e}\end{array}$ & $\mathrm{s}$ & \\
\hline $\begin{array}{l}\text { local volumetric } \\
\text { rate of energy } \\
\text { absorption, LVREA }\end{array}$ & $L_{\lambda}^{\mathrm{a}}(\boldsymbol{x}, t)$ & $\begin{array}{l}L_{\lambda}^{\mathrm{a}}(\boldsymbol{x}, t)=\alpha_{\lambda}(\boldsymbol{x}, t) E_{\lambda, \mathrm{o}}(\boldsymbol{x}, t) \\
E_{\lambda, \mathrm{o}}(\boldsymbol{x}, t) \text { is the space- and time-dependent } \\
\text { spectral fluence rate incident from all } \\
\text { directions at } \boldsymbol{x}, \alpha_{\lambda}(\boldsymbol{x}, t) \text { is the } \\
\text { space- and time-dependent linear } \\
\text { napierian absorption coefficient. }\end{array}$ & $\mathrm{W} \cdot \mathrm{m}^{-4}$ & 4,27 \\
\hline $\begin{array}{l}\text { local volumetric } \\
\text { rate of photon } \\
\text { absorption, LVRPA }\end{array}$ & $L_{\mathrm{p}, \lambda}^{\mathrm{a}}(\boldsymbol{x}, t)$ & $\begin{array}{l}L_{\mathrm{p}, \lambda}^{\mathrm{a}}(\boldsymbol{x}, t)=\alpha_{\lambda}(\boldsymbol{x}, t) E_{\mathrm{p}, \lambda, \mathrm{o}}(\boldsymbol{x}, t) \\
\text { with } E_{\mathrm{p}, \lambda, \mathrm{o}}(\boldsymbol{x}, t)=\int_{4 \pi} L_{\mathrm{p}, \lambda}(\boldsymbol{x}, t) \mathrm{d} \Omega \\
\text { the spectral photon fluence rate incident } \\
\text { from all directions at } \boldsymbol{x}, \text { and } \alpha_{\lambda}(\boldsymbol{x}, t) \text { the } \\
\text { space- and time-dependent linear } \\
\text { napierian absorption coefficient. }\end{array}$ & $\mathrm{s}^{-1} \cdot \mathrm{m}^{-4}$ & 3,28 \\
\hline mass concentration & $\rho$ & $\rho=m / V$ & $\mathrm{~kg} \cdot \mathrm{m}^{-3}$ & 29 \\
\hline $\begin{array}{l}\text { number density } \\
\text { of entities, } \\
\text { number } \\
\text { concentration }\end{array}$ & $C, n$ & $C=n=N / V$ & $\mathrm{~m}^{-3}$ & \\
\hline $\begin{array}{l}\text { number of } \\
\text { photoelectrons }\end{array}$ & $N_{\mathrm{e}}$ & $\begin{array}{l}N_{\mathrm{e}}=g(x) \tau_{\mathrm{e}} \\
\text { with } g(x) \text { the rate of photogeneration of } \\
\text { carriers and } \tau_{\mathrm{e}} \text { the lifetime of the free } \\
\text { electrons. }\end{array}$ & 1 & \\
\hline $\begin{array}{l}\text { number of } \\
\text { photoholes }\end{array}$ & $N_{\mathrm{h}}$ & $\begin{array}{l}N_{\mathrm{h}}=g(x) \tau_{\mathrm{h}} \\
\text { with } g(x) \text { the rate of photogeneration of } \\
\text { carriers and } \tau_{\mathrm{h}} \text { the lifetime of the free } \\
\text { holes. }\end{array}$ & 1 & \\
\hline out-scattering & $\mathrm{Sc}_{\text {out }}$ & $\begin{array}{l}\mathrm{Sc}_{\text {out }}=\sigma_{\lambda}(\boldsymbol{x}, t) L_{\Omega, \lambda}(\boldsymbol{x}, t) \\
\sigma_{\lambda}(\boldsymbol{x}, t) \text { is the space- and time-dependent } \\
\text { napierian scattering coefficient and } \\
L_{\Omega, \lambda}(\boldsymbol{x}, t) \text { the incident space- and } \\
\text { time-dependent radiance. }\end{array}$ & & 25 \\
\hline
\end{tabular}


(Continued)

\begin{tabular}{llllc}
\hline Name & Symbol & \multicolumn{1}{c}{ Definition } & SI unit & Notes \\
\hline $\begin{array}{l}\text { photoadsorption } \\
\text { area number }\end{array}$ & $\gamma^{\mathrm{S}}$ & $\begin{array}{l}\gamma^{\mathrm{S}}=N_{\max } / S \\
\text { with } N_{\max } \text { the maximal number of } \\
\text { photoadsorbed entities and } S \text { the } \\
\text { surface area. }\end{array}$ & $\begin{array}{l}\mathrm{m}^{-2} \text { or } \\
\mathrm{mol} \cdot \mathrm{m}^{-2}\end{array}$ & 30 \\
& & &
\end{tabular}

photoadsorption

$\theta_{\mathrm{m}}$

$\theta_{\mathrm{m}}=N_{\max } / N^{\mathrm{ss}}$

capacity

with $N_{\max }$ the maximal number of photoadsorbed entities and $N^{\text {ss }}$ the number of surface sites.

photoadsorption

$\theta_{\mathrm{ph}}$

$\theta_{\mathrm{ph}}=N / N^{\mathrm{ss}}$

fraction

with $N$ the number of photoadsorbed

(photoadsorption

number)

entities and $N^{\mathrm{ss}}$ the number of surface

sites.

photoadsorption

efficiency

(of entities B)

photoadsorption

photonic yield

$P_{\mathrm{AE}} \quad P_{\mathrm{AE}}=\frac{\mathrm{d} N_{\mathrm{B}} / \mathrm{d} t}{\int_{\lambda_{1}}^{\lambda_{2}} q_{\mathrm{p}, \lambda}^{0} \mathrm{~d} \lambda}$

with $N_{\mathrm{B}}$ the number of adsorbed entities

$\mathrm{B}$ and the incident spectral photon flux, both at initial rates (for $t \rightarrow 0$ ).

$\mathrm{d} N_{\mathrm{B}}$

$\frac{\mathrm{d} t}{q_{\mathrm{p}, \lambda}^{0}}$

with $N_{\mathrm{B}}$ the number of adsorbed entities $\mathrm{B}$ and $q_{\mathrm{p}, \lambda}^{0}$ the incident spectral photon flux, both as initial rates (for $t \rightarrow 0$ ).

photoadsorption quantum yield

$$
\Phi_{\text {ads }} \quad \Phi_{\mathrm{ads}}=\frac{\mathrm{d} N_{\mathrm{B}} / \mathrm{d} t}{\mathrm{~d} N_{\mathrm{p}, \lambda} / \mathrm{d} t}
$$

with $N_{\mathrm{B}}$ the number of adsorbed entities B and $N_{\mathrm{p}, \lambda}$ the number of photons of a particular wavelength absorbed, both as initial rates (for $t \rightarrow 0$ ).

photodesorption quantum yield $\Phi_{\mathrm{des}} \quad \Phi_{\mathrm{des}}=\frac{\mathrm{d} N_{\mathrm{des}} / \mathrm{d} t}{q_{\mathrm{p}, \lambda}}$ 1 (differential)

with $N_{\text {des }}$ the maximal desorption rate and $q_{\mathrm{p}, \lambda}$ the spectral photon flux absorbed. 
(Continued)

\begin{tabular}{|c|c|c|c|c|}
\hline Name & Symbol & Definition & SI unit & Notes \\
\hline $\begin{array}{l}\text { photodesorption } \\
\text { quantum yield } \\
\text { (integral) }\end{array}$ & $\Phi_{\mathrm{des}}$ & $\begin{array}{l}\Phi_{\mathrm{des}}=\frac{N_{\mathrm{des}}}{N_{\mathrm{p}, \lambda}} \\
\text { with } N_{\mathrm{des}} \text { the number of enities desorbed } \\
\text { and } N_{\mathrm{p}, \lambda} \text { the number of photons of a } \\
\text { particular wavelength absorbed during } \\
\text { a given irradiation time. }\end{array}$ & & 1 \\
\hline photon fluence & $F_{\mathrm{p}, \mathrm{o}}$ & $F_{\mathrm{p}, \mathrm{o}}=\mathrm{d} N_{\mathrm{p}} / \mathrm{d} S$ & $\mathrm{~m}^{-2}$ & 22,31 \\
\hline photon fluence rate & $E_{\mathrm{p}, \mathrm{o}}$ & $\begin{array}{l}E_{\mathrm{p}, \mathrm{o}}=\mathrm{d} F_{\mathrm{p}, \mathrm{o}} / \mathrm{d} t \\
\text { For a divergent beam } \\
E_{\mathrm{p}, \lambda, \mathrm{o}}(\boldsymbol{x}, t)=\int_{\Omega} L_{\mathrm{p}, \lambda}(\boldsymbol{x}, t, \Omega) \mathrm{d} \Omega\end{array}$ & $\mathrm{m}^{-2} \cdot \mathrm{s}^{-1}$ & 22,31 \\
\hline $\begin{array}{l}\text { photon flux, } \\
\text { amount basis }\end{array}$ & $q_{n, \mathrm{p}}$ & $q_{n, \mathrm{p}}=q_{\mathrm{p}} / N_{\mathrm{A}}=\left(\mathrm{d} N_{\mathrm{p}} / t\right) / N_{\mathrm{A}}$ & $\mathrm{mol} \cdot \mathrm{s}^{-1}$ & 32 \\
\hline $\begin{array}{l}\text { photon flux, } \\
\text { number basis }\end{array}$ & $q_{\mathrm{p}}$ & $\mathrm{d} N_{\mathrm{p}} / \mathrm{d} t$ & $\mathrm{~s}^{-1}$ & 31,33 \\
\hline photon irradiance & $E_{\mathrm{p}}$ & $E_{\mathrm{p}}=\mathrm{d} q_{\mathrm{p}} / \mathrm{d} S$ & $\mathrm{~m}^{-2} \cdot \mathrm{s}^{-1}$ & 31,34 \\
\hline photon number & $N_{\mathrm{p}}$ & $N_{\mathrm{p}}=n_{\mathrm{p}} N_{\mathrm{A}}$ & 1 & \\
\hline \multirow[t]{2}{*}{ photon radiance } & $L_{\mathrm{p}}$ & $\begin{array}{l}\text { for a parallel beam } \\
L_{\mathrm{p}}=\left(\mathrm{d} q_{\mathrm{p}} / \mathrm{d} S\right) / \cos \theta \\
\text { for a } \underline{\text { divergent beam propagating in an }} \\
\text { elementary cone of solid angle } \Omega \\
\text { containing the beam direction } \theta \text { in a } \\
\text { three-dimensional space. }\end{array}$ & $\mathrm{m}^{-2} \cdot \mathrm{s}^{-1}$ & 35 \\
\hline & & $L_{\mathrm{p}, \Omega}=\lim _{(\Delta \Omega, \Delta S, \Delta t) \rightarrow 0} \frac{\Delta N_{\mathrm{P}}}{\Delta \Omega \Delta S \cos \theta \Delta t}$ & $\mathrm{~m}^{-2} \cdot \mathrm{s}^{-1} \cdot \mathrm{sr}^{-1}$ & \\
\hline \multirow[t]{2}{*}{ photonic efficiency } & $\xi$ & $\xi=\frac{\mathrm{d} n / \mathrm{d} t}{\int_{\lambda_{1}}^{\lambda_{2}} q_{n, \mathrm{p}, \lambda}^{0}}$ in chemical amount basis & 1 & 36 \\
\hline & & $\begin{array}{l}\xi=\frac{\mathrm{d} N / \mathrm{d} t}{\int_{\lambda_{1}}^{\lambda_{2}} q_{\mathrm{p}, \lambda}^{0}} \text { in number basis } \\
q_{n, \mathrm{p}, \lambda}^{0} \text { is the spectral photon } \\
\text { flux (amount basis) and } q_{\mathrm{p}, \lambda}^{0} \text { is the } \\
\text { spectral photon flux (number basis) } \\
\text { incident per time interval within a } \\
\text { defined wavelength range. }\end{array}$ & 1 & \\
\hline
\end{tabular}


(Continued)

\begin{tabular}{|c|c|c|c|c|}
\hline Name & Symbol & Definition & SI unit & Notes \\
\hline photonic yield & & $\begin{array}{l}\frac{\mathrm{d} n / \mathrm{d} t}{q_{n, \mathrm{p}, \lambda}^{0}} \text { in chemical amount basis } \\
\frac{\mathrm{d} N / \mathrm{d} t}{q_{\mathrm{p}, \lambda}^{0}} \text { in number basis } \\
q_{n, \mathrm{p}, \lambda}^{0} \text { is the spectral photon flux, chemical } \\
\text { amount basis incident per time interval, } \\
n \text { the amount of substance produced or } \\
\text { consumed, } q_{\mathrm{p}, \lambda}^{0} \text { the incident spectral } \\
\text { photon flux, number basis, and } N \text { the } \\
\text { number of entities produced or consumed. }\end{array}$ & 1 & 37 \\
\hline Planck constant & $\begin{array}{l}h \\
\hbar=h / 2 \pi\end{array}$ & $\begin{array}{l}6.62606896(33) \times 10^{-34} \\
1.054571628(53) \times 10^{-34}\end{array}$ & $\begin{array}{l}J \cdot \mathrm{s} \\
\mathrm{J} \cdot \mathrm{s}\end{array}$ & $\begin{array}{l}15 \\
15\end{array}$ \\
\hline $\begin{array}{l}\text { post-adsorption } \\
\text { memory coefficient }\end{array}$ & $K(t)$ & $\begin{array}{l}K(t)=\frac{N_{\text {post }}(t)}{N_{\text {phot }}(t)} \\
\text { with } N_{\text {post }}(t) \text { the number of entities } \\
\text { adsorbed after termination of } \\
\text { irradiation time, } t \text {, and } N_{\text {phot }}(t) \text { the } \\
\text { number of photoadsorbed molecules } \\
\text { during the same time. }\end{array}$ & & 38 \\
\hline pressure & $P, p$ & & $\mathrm{~Pa}$ & \\
\hline \multirow[t]{2}{*}{ quantum efficiency } & $\Phi(\Delta \lambda)$ & $\begin{array}{l}\Phi(\Delta \lambda)=\frac{\int_{\lambda_{1}}^{\lambda_{2}} \frac{\mathrm{d} x(\lambda)}{\mathrm{d} t} \mathrm{~d} \lambda}{\int_{\lambda_{1}}^{\lambda_{2}} q_{n, \mathrm{p}, \lambda}^{0}\left[1-10^{-A}\right] \mathrm{d} \lambda} \\
\text { for an homogeneous system and within } \\
\text { a defined wavelength range. } A \text { is the } \\
\text { decadic absorbance at wavelength } \lambda, x \\
\text { is a wavelength-dependent quantity } \\
\text { measuring the reaction process. } \\
\text { For a divergent beam: }\end{array}$ & 1 & 36,39 \\
\hline & & $\Phi(\Delta \lambda)=\frac{\int_{\lambda_{1}}^{\lambda_{2}}[\mathrm{~d} x(\lambda) / \mathrm{d} t] \mathrm{d} \lambda}{\int_{\lambda_{1}}^{\lambda_{2}}\left[\int_{V} \alpha_{\lambda}(\boldsymbol{x}, t) E_{n, \mathrm{p}, \lambda_{\mathrm{o}}}(\boldsymbol{x}, t) \mathrm{d} V\right] \mathrm{d} \lambda}$ & 1 & \\
\hline $\begin{array}{l}\text { quantum yield } \\
\text { (integral) }\end{array}$ & $\Phi$ & $\begin{array}{l}\Phi=(\text { number of events }) /(\text { number of } \\
\text { photons absorbed) for monochromatic } \\
\text { excitation }\end{array}$ & 1 & 40 \\
\hline
\end{tabular}


(Continued)

\begin{tabular}{|c|c|c|c|c|}
\hline Name & Symbol & Definition & SI unit & Notes \\
\hline $\begin{array}{l}\text { quantum yield } \\
\text { (average) of a } \\
\text { photocatalytic }\end{array}$ & $\Phi_{\mathrm{Ph} \rightarrow \text { cat }}$ & $\Phi_{\mathrm{Ph} \rightarrow \text { cat }}(\lambda)=\frac{\mathrm{d} x / \mathrm{d} t}{\left\langle L_{\mathrm{p}, \lambda}^{a}(t)\right\rangle_{V}}$ & 1 & 41 \\
\hline $\begin{array}{l}\text { process in } \\
\text { technical } \\
\text { photoreactors }\end{array}$ & & $\begin{array}{l}\left\langle L_{\mathrm{p}, \lambda}^{\mathrm{a}}(t)\right\rangle_{V} \text { is the absorbed (spectral) } \\
\text { photon flux density and } x \text { a measure of } \\
\text { concentration of product formation or } \\
\text { reactant consumed. }\end{array}$ & & \\
\hline radiance & $L_{\Omega}$ & $L_{\Omega}=\lim _{(\Delta \Omega, \Delta S, \Delta t) \rightarrow 0} \frac{\Delta P}{\Delta \Omega \Delta S \cos \theta}$ & $\mathrm{W} \cdot \mathrm{m}^{-2} \cdot \mathrm{sr}^{-1}$ & \\
\hline radiant energy & $Q, W$ & $Q=\int_{\lambda} Q_{\lambda} \mathrm{d} \lambda$ & $\mathrm{J}$ & \\
\hline radiant intensity & $I$ & $I=\mathrm{d} P / \mathrm{d} \Omega$ & $\mathrm{W} \cdot \mathrm{sr}^{-1}$ & \\
\hline $\begin{array}{l}\text { radiant power, } \\
\text { radiant energy } \\
\text { per time interval }\end{array}$ & $P$ & $P=\mathrm{d} Q / \mathrm{d} t$ & W & 42 \\
\hline $\begin{array}{l}\text { radiation chemical } \\
\text { yield }\end{array}$ & $G$ & $\begin{array}{l}\left(N_{\mathrm{B}} / Q_{\mathrm{e}}\right) \times 100 \\
N_{\mathrm{B}} \text { is the number of entities B formed } \\
\text { (or disappeared) } Q_{\mathrm{ev}} \text { is the energy in } \mathrm{eV} \text {. } \\
\text { Amount of substance B (in } \mu \text { mol) } \\
\text { produced per J of energy absorbed }\end{array}$ & $\mu \mathrm{mol} \cdot \mathrm{J}^{-1}$ & 43 \\
\hline $\begin{array}{l}\text { rate of } \\
\text { (photo)generation } \\
\text { of carriers }\end{array}$ & $g(x)$ & $\begin{array}{l}g(x)=\alpha E_{\mathrm{p}}(1-\rho) \exp (-\alpha x) \\
\alpha \text { is the linear napierian absorption } \\
\text { coefficient, } E_{\mathrm{p}} \text { the photon irradiance } \\
\text { and } \rho \text { the reflectance }\end{array}$ & $\begin{array}{l}\mathrm{m}^{-3} \cdot \mathrm{s}^{-1} \text { or } \\
\mathrm{mol}^{-1} \cdot \mathrm{m}^{-3} \cdot \mathrm{s}^{-1}\end{array}$ & 44 \\
\hline $\begin{array}{l}\text { rate of surface } \\
\text { recombination }\end{array}$ & $s$ & $\begin{array}{l}s=1 / \tau_{\mathrm{fc}} \\
\text { with } \tau_{\mathrm{fc}} \text { the lifetime of free carriers. }\end{array}$ & $\mathrm{s}^{-1}$ & 45 \\
\hline $\begin{array}{l}\text { reflectance, } \\
\text { reflectivity }\end{array}$ & $\rho$ & $\begin{array}{l}\rho=P_{\lambda}^{\text {refl }} / P_{\lambda}^{0} \\
\rho(\lambda)=\frac{\left(n_{1}-n_{2}\right)^{2}}{\left(n_{1}+n_{2}\right)^{2}} \\
\text { with } n_{1} \text { and } n_{2} \text { the refractive index of } \\
\text { each of the media. }\end{array}$ & 1 & 46 \\
\hline refractive index & $n$ & $\begin{array}{l}n=c_{0} / c \\
\text { with } c_{0} \text { and } c \text { the velocity of light in } \\
\text { vacuum and in the medium, respectively. }\end{array}$ & 1 & \\
\hline
\end{tabular}


(Continued)

\begin{tabular}{|c|c|c|c|c|}
\hline Name & Symbol & Definition & SI unit & Notes \\
\hline $\begin{array}{l}\text { relative photonic } \\
\text { efficiency }\end{array}$ & $\xi_{\text {rel }}$ & & 1 & 47 \\
\hline scattering albedo & & $\begin{array}{l}\sigma(\lambda) / \beta(\lambda) \\
\text { with } \sigma(\lambda) \text { the napierian scattering } \\
\text { coefficient and } \beta(\lambda) \text { the napierian } \\
\text { extinction coefficient, both at the same } \\
\text { wavelength. }\end{array}$ & 1 & \\
\hline \multicolumn{5}{|l|}{ scattering coefficient } \\
\hline $\begin{array}{l}\text { linear napierian } \\
\text { specific napierian }\end{array}$ & $\begin{array}{l}\sigma(\lambda) \\
\sigma^{*}(\lambda)\end{array}$ & $\begin{array}{l}\sigma(\lambda)=\left[D_{\mathrm{e}}(\lambda)-A_{\mathrm{e}}(\lambda)\right] / l \\
\sigma^{*}(\lambda)=\left[D_{\mathrm{e}}(\lambda)-A_{\mathrm{e}}(\lambda)\right] / \rho l \\
\text { with } \rho \text { the mass concentration. }\end{array}$ & $\begin{array}{l}\mathrm{m}^{-1} \\
\mathrm{~m}^{2} \cdot \mathrm{kg}^{-1}\end{array}$ & $\begin{array}{l}48 \\
10\end{array}$ \\
\hline solid angle & $\Omega$ & $\Omega=S / r^{2}$ & sr, 1 & 49 \\
\hline \multicolumn{5}{|l|}{ specific (spectral) } \\
\hline napierian & $\alpha *(\lambda)$ & $\alpha^{*}(\lambda)=\frac{\alpha(\lambda)}{\rho}$ & $\mathrm{m} \cdot \mathrm{kg}^{-1}$ & $\begin{array}{c}10,50, \\
51\end{array}$ \\
\hline decimal & $a^{*}(\lambda)$ & $\begin{array}{l}a *(\lambda)=\frac{a(\lambda)}{\rho} \\
\quad \text { with } \rho \text { the mass concentration. }\end{array}$ & & \\
\hline $\begin{array}{l}\text { specific napierian } \\
\text { (spectral) attenuance } \\
\text { coefficient }\end{array}$ & $\beta^{*}(\lambda)$ & $\begin{array}{l}\beta^{*}(\lambda)=\beta(\lambda) / \rho \\
\text { with } \rho \text { the mass concentration. } \\
\beta^{*}(\lambda)=\alpha^{*}(\lambda)+\sigma^{*}(\lambda)\end{array}$ & $\mathrm{m}^{2} \cdot \mathrm{kg}^{-1}$ & $\begin{array}{c}10,50, \\
51\end{array}$ \\
\hline $\begin{array}{l}\text { specific napierian } \\
\text { (spectral) scattering } \\
\text { coefficient }\end{array}$ & $\sigma^{*}(\lambda)$ & $\begin{array}{c}\sigma^{*}(\lambda)=\sigma(\lambda) / \rho=\frac{\left[D_{\mathrm{e}}(\lambda)-A_{\mathrm{e}}(\lambda)\right]}{\rho l} \\
\text { with } \rho \text { the mass concentration. }\end{array}$ & $\mathrm{m}^{2} \cdot \mathrm{kg}^{-1}$ & $\begin{array}{c}10,48, \\
51\end{array}$ \\
\hline $\begin{array}{l}\text { specific surface } \\
\text { area }\end{array}$ & $a^{\mathrm{s}}$ & $a^{\mathrm{s}}=S / \mathrm{m}$ & $\mathrm{m}^{2} \cdot \mathrm{kg}^{-1}$ & 52 \\
\hline $\begin{array}{l}\text { spectral distribution } \\
\text { (of a radiant, luminous } \\
\text { or photon quantity) }\end{array}$ & $X_{\lambda}(\lambda)$ & $X_{\lambda}(\lambda)=\mathrm{d} X(\lambda) / \mathrm{d} \lambda$ & {$[X] \cdot \mathrm{m}^{-1}$} & \\
\hline $\begin{array}{l}\text { spectral fluence } \\
\text { (in terms of } \\
\text { wavelength) }\end{array}$ & $F_{\lambda, \mathrm{o}}$ & $F_{\lambda, \mathrm{o}}=\mathrm{d} F_{\mathrm{o}} / \mathrm{d} \lambda$ & $\mathrm{J} \cdot \mathrm{m}^{-3}$ & 21,53 \\
\hline
\end{tabular}


(Continued)

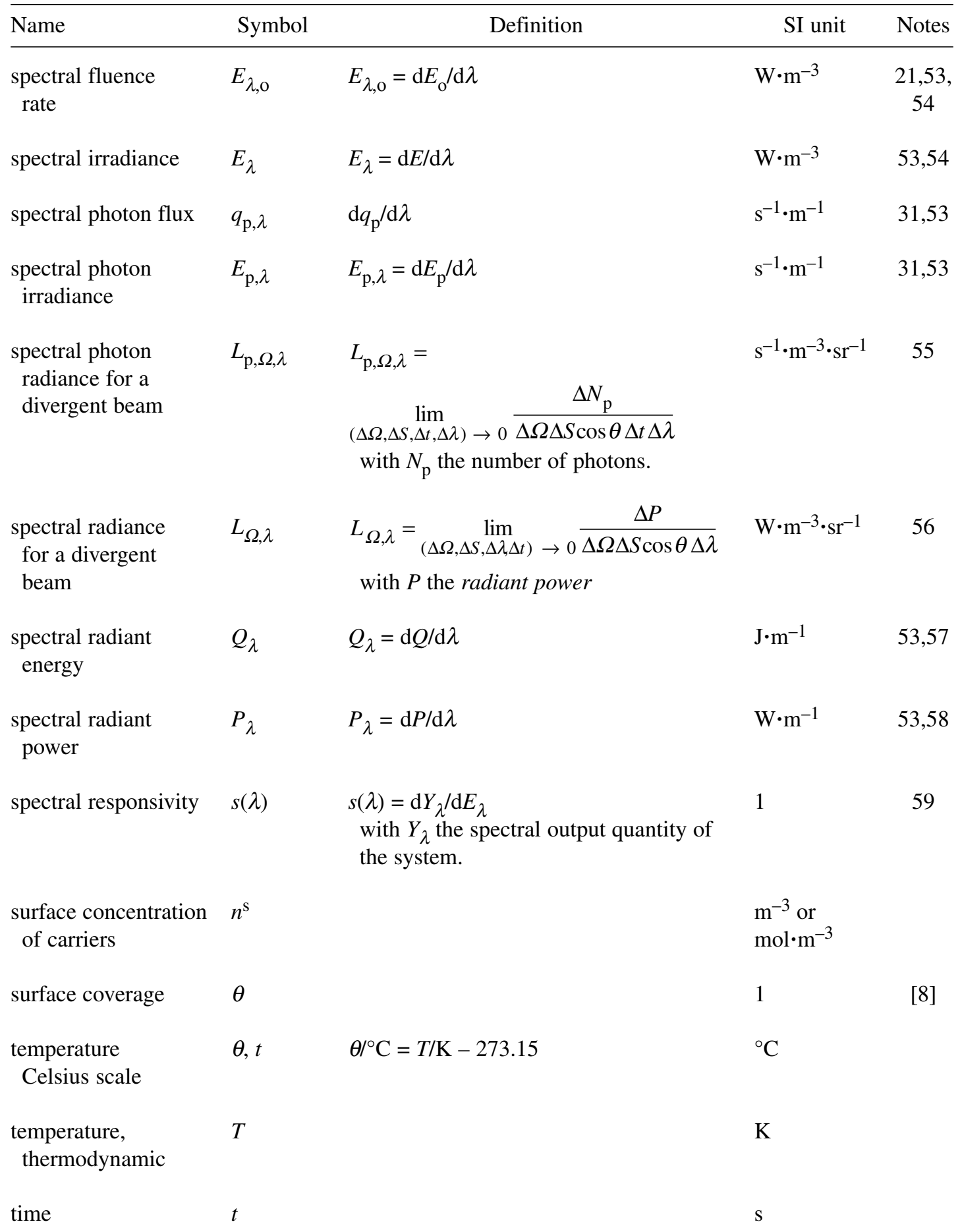


(Continued)

\begin{tabular}{|c|c|c|c|c|}
\hline Name & Symbol & Definition & SI unit & Notes \\
\hline transmittance & $T$ & $\begin{array}{l}T=P_{\lambda} / P_{\lambda}^{0} \\
\text { with } P_{\lambda}^{0} \text { and } P_{\lambda} \text { the spectral radiant power } \\
\text { incident (superscript } 0 \text {, zero) and } \\
\text { transmitted (no superscript), respectively. }\end{array}$ & 1 & 2 \\
\hline $\begin{array}{l}\text { turnover frequency } \\
\text { (TOF), } \\
\text { turnover rate (TOR) }\end{array}$ & $\tau_{\mathrm{F}}$ & $\begin{array}{l}\tau_{\mathrm{F}}=\frac{1}{N_{\mathrm{a}}} \frac{\mathrm{d} N}{\mathrm{~d} t} \\
\text { with } N_{\mathrm{a}} \text { the number of catalyst active sites } \\
\text { and } N \text { the number of catalytic events. }\end{array}$ & $\mathrm{s}^{-1}$ & 60 \\
\hline $\begin{array}{l}\text { turnover frequency } \\
\text { in heterogeneous } \\
\text { photocatalysis }\end{array}$ & $\tau_{\text {het }}$ & $\begin{array}{l}\tau_{\text {het }}=\frac{1}{S} \frac{\mathrm{d} N}{\mathrm{~d} t} \\
\text { with } S=\operatorname{BET}\left(\mathrm{N}_{2}\right)\end{array}$ & $\mathrm{s}^{-1} \cdot \mathrm{m}^{-2}$ & 61 \\
\hline $\begin{array}{l}\text { turnover frequency } \\
\text { in homogeneous } \\
\text { photocatalysis }\end{array}$ & $\tau_{\text {hom }}$ & $\begin{array}{l}\tau_{\text {hom }}=\frac{1}{C_{\mathrm{m}}} \frac{\mathrm{d} N}{\mathrm{~d} t} \\
\text { with } C_{\mathrm{m}} \text { the number concentration of the } \\
\text { photocatalyst. }\end{array}$ & $\mathrm{s}^{-1}$ & \\
\hline $\begin{array}{l}\text { turnover number } \\
\text { TON }\end{array}$ & & & 1 & \\
\hline volume & $V$ & & $\mathrm{~m}^{3}$ & \\
\hline wavelength & $\lambda$ & & $\mathrm{m}$ & \\
\hline $\begin{array}{l}\text { wavenumber in } \\
\text { vacuum }\end{array}$ & $\widetilde{v}$ & $\begin{array}{l}\widetilde{v}=v / c_{0}=1 / n \lambda \\
\text { with } n \text { the refractive index of the medium. }\end{array}$ & $\mathrm{m}^{-1}$ & \\
\hline
\end{tabular}

\section{NOTES}

(1) Entries in the table are consistent with terminology, symbols, and units given in [8] and sometimes incompatible with those in [9], and are slightly modified from those in [68], terms from which had been incorporated in [8]. Symbols individually listed in the Table may not be explained within a definition.

(2) If losses from reflection, scattering and luminescence are neglected, $T=P / P^{0}=I / I^{0}$, where superscript 0 (zero) indicates incident radiant power (or intensity) and no superscript transmitted radiant power (or intensity). Transmittance depends on pathlength, which should be specified. In common usage $A$ is given for $1 \mathrm{~cm}$ pathlength unless otherwise specified [7].

(3) Common unit is $\mathrm{s}^{-1} \cdot \mathrm{cm}^{-3} \cdot \mathrm{nm}^{-1}$ in photon number basis and einstein $\cdot \mathrm{s}^{-1} \cdot \mathrm{cm}^{-3} \cdot \mathrm{nm}^{-1}$ in chemical amount basis, although einstein (E) is not an accepted SI unit. Absorption coefficients are always spectral and the subscript $\lambda$ may be omitted. The wavelength should be specified, e.g., $\alpha(300 \mathrm{~nm})$.

(4) Common unit is $\mathrm{W} \cdot \mathrm{cm}^{-3} \cdot \mathrm{nm}^{-1}$. Absorption coefficients are always spectral and the subscript $\lambda$ may be omitted, but the wavelength should be specified, e.g., $\alpha(300 \mathrm{~nm})$. 
(5) Average (over total volume) of the absorbed local spectral photon flux density. $\boldsymbol{x}$ defines a position in a three-dimensional space and $\alpha^{*}$ is the specific napierian absorption coefficient. Absorption coefficients are always spectral and the subscript $\lambda$ may be omitted, but the wavelength should be specified, e.g., $\alpha(300 \mathrm{~nm})$. Common unit is $\mathrm{s}^{-1} \cdot \mathrm{cm}^{-3} \cdot \mathrm{nm}^{-1}$ in photon number basis and einstein $\cdot \mathrm{s}^{-1} \cdot \mathrm{cm}^{-3} \cdot \mathrm{nm}^{-1}$ in chemical amount basis.

(6) Common unit is $\mathrm{W} \cdot \mathrm{cm}^{-3} \cdot \mathrm{nm}^{-1}$.

(7) Term in the radiative transfer equation (RTE). Common unit is $\mathrm{W} \cdot \mathrm{cm}^{-3} \cdot \mathrm{sr}^{-1} \cdot \mathrm{nm}^{-1}$.

(8) In spectroscopy, usually defined in terms of the spectral radiant power, $P_{\lambda}$.

(9) Numerical values are often quoted in $\mathrm{mol}^{-1} \cdot \mathrm{dm}^{3} \cdot \mathrm{cm}^{-1}$. Note the lack of compactness in using two submultiples of length. All absorption coefficients depend on wavelength, or wavenumber, or frequency and may be quoted as $\varepsilon(\lambda)$, or as $\varepsilon(\widetilde{v})$, or as $\varepsilon(v)$.

(10) Absorption (as well as attenuance and scattering) coefficient with superscript* is utilised when the molecular weight of the irradiated substance is unknown. These coefficients are called specific coefficients. Common unit is $\mathrm{dm}^{3} \cdot \mathrm{g}^{-1} \cdot \mathrm{cm}^{-1}$. Note the lack of compactness in using two submultiples of length. Absorption coefficient is a spectral property. The subscript $\lambda$ may be omitted. The wavelength should be specified, e.g., $\alpha *(300 \mathrm{~nm})$.

(11) In spectroscopy, the net cross-section resulting from the sum of effects due to absorption and induced emission. A conversion equation in common units is $\sigma / \mathrm{cm}^{2}=\left(3.8236 \times 10^{-21} \mathrm{~mol}^{-1}\right) \times$ $\left(\varepsilon / \mathrm{mol}^{-1} \cdot \mathrm{dm}^{3} \cdot \mathrm{cm}^{-1}\right)$.

(12) Amount of photons is often given in the non-SI unit einstein [7].

(13) Usual units are $\mathrm{mol} \cdot \mathrm{dm}^{-3}$ or $\mathrm{mol} \cdot \mathrm{L}^{-1}$ or submultiples. Commonly the non-SI unit M (small cap) is used as an abbreviation for $\mathrm{mol} \cdot \mathrm{dm}^{-3}$. B designates an entity.

(14) Attenuance reduces to absorbance for a not reflected or scattered beam [7]. Measurements of attenuance combined with experiments with integrating spheres permit to separate scattering from absorption processes.

(15) 2006 value [69].

(16) Common non-SI unit is electron volt $(\mathrm{eV})=1.60217653(14) \times 10^{-19} \mathrm{~J}$; for one mole of electrons $1 \mathrm{eV}$ corresponds to $96.485 \mathrm{~kJ} \cdot \mathrm{mol}^{-1}$.

(17) The most common gas used is $\mathrm{N}_{2}$ with a molecular cross-section of $0.162 \mathrm{~nm}^{2}$, in which case the surface area is called BET $\left(\mathrm{N}_{2}\right)$. The properties of a photocatalyst are not directly proportional to their area, in view of the fact that light does not equally reach every part of the surface or particle. Common unit is $\mathrm{m}^{2} \cdot \mathrm{g}^{-1}$.

(18) Defined in [8]. Commonly given in $\mathrm{cm}^{2}$. In photocatalysis, a quantum yield $\Phi$ is difficult to determine. Photonic yield, i.e., in terms of incident radiation, is a more appropriate quantity.

(19) SI unit is siemens per $\mathrm{m}^{-1}[9]$.

(20) The term extinction should be reserved to scattering and dispersive media.

(21) For a beam not scattered or reflected by the sample a synonym is radiant exposure, $H$. When applied to the total radiant energy incident from all directions the symbol $H_{\mathrm{o}}$ or $F_{\mathrm{o}}$ is used.

(22) The symbols related to radiant energy incident from all directions have a subscript o (the letter o), not to be confused with supescript 0 (zero) for incident radiation (prior to absorption, footnote 1).

(23) For a parallel and normally incident beam a synonym is irradiance. When applied to the total radiant energy incident from all directions the symbol $E_{\mathrm{o}}$ is used.

(24) For photocatalytic reactions initial rates must be measured, i.e., for $t \rightarrow 0$.

(25) One of the terms of the RTE. Definition valid for multiple, independent, and elastic scattering. $x$ is the spatial coordinate in a three-dimensional space along the propagation of the beam, $t$ is the time coordinate, $\Omega$ is the direction of the rays, $\sigma_{\lambda}(x, t)$ is the space- and time-dependent napierian linear scattering coefficient, $L_{\Omega, \lambda}(\boldsymbol{x}, t)$ is the incident space- and time-dependent radiance. Each of the terms in the RTE may be expressed in photon terms.

(26) The corresponding term for a beam incident from all directions is fluence rate $\left(E_{\mathrm{o}}\right)$.

(27) Same as absorbed local spectral radiance.

(C) 2011, IUPAC

Pure Appl. Chem., Vol. 83, No. 4, pp. 931-1014, 2011 
(28) Same as absorbed local spectral photon flux density.

(29) Defined in [8]. Common unit is $\mathrm{g} \cdot \mathrm{cm}^{-3}$.

(30) In photocatalysis, the area-related parameters do not characterise the catalyst as they do in heterogeneous dark catalysis. The properties of a photocatalyst are not directly proportional to their area, in view of the fact that light does not equally reach every part of a surface or particle.

(31) These quantities, defined on a number basis, can be expressed on a chemical amount basis by dividing by the Avogadro constant, e.g., photon flux (chemical amount basis) $q_{n, \mathrm{p}}=q_{\mathrm{p}} / N_{\mathrm{A}}$. If distinction needs to be made between quantities based on chemical amount and number, then symbols such as $q_{n, \mathrm{p}}$ and $q_{\mathrm{p}}$ can be used.

(32) Common unit is einstein $\cdot \mathrm{s}^{-1}$. A term not in accordance with the usual definition of flux [9].

(33) As defined in [8], called photon flux in [59], a term not in accordance with the usual definition of flux [9].

(34) The corresponding term for a beam incident from all directions is photon fluence rate $\left(E_{\mathrm{p}, \mathrm{o}}\right)$.

(35) The photon quantities, defined on a number basis, can be expressed on a chemical amount basis by dividing by the Avogadro constant, e.g., photon flux (chemical amount basis) $q_{n, \mathrm{p}}=q_{\mathrm{p}} / N_{\mathrm{A}}$. Should distinction be made between quantities based on chemical amount and number, then symbols such as $q_{n, \mathrm{p}}$ and $q_{\mathrm{p}}$ may be used. The relationship between a photon quantity (e.g., photon flux $q_{\mathrm{p}}$ ) and a wavelength-dependent radiometric quantity [e.g., radiant power $P(\lambda)$ ] integrated over a wavelength range $\lambda_{1}-\lambda_{2}$ is given by $q_{\mathrm{p}}=1 /(h c) \int_{\lambda_{1}}^{\lambda_{2}} P(\lambda) \lambda \mathrm{d} \lambda$. Thus, to calculate a photon flux over a wavelength interval, the spectral distribution of the radiant power is necessary. Note the relationship between spectral, radiometric, and photon terms as developed in the Introduction.

(36) Superscript 0 (zero) means that the incident number of photons must be used. Efficiencies in heterogeneous photocatalysis are measured within a defined wavelength interval (polychromatic excitation).

(37) Quantum and photonic yields are for monochromatic excitation.

(38) $K$ is a function of the time of pre-irradiation.

(39) This definition applies especially for polychromatic excitation. In homogeneous photochemistry and when the quantum yield is independent of excitation wavelength in the spectral region used, quantum efficiency is identical to quantum yield.

(40) Strictly, the term quantum yield applies only for monochromatic excitation $(\lambda+d \lambda)$.

(41) Needed for large photochemical reactors with heterogeneous distribution of radiation and large scattering.

(42) Definition from [9]; synonym with radiant energy power, radiant energy flux defined in [58]. The alternative symbol $\Phi$ is not recommended in connection with photochemistry or photocatalysis, because $\Phi$ is reserved for quantum yield.

(43) $G$ values have been traditionally expressed in $(100 \mathrm{eV})^{-1}$. Using the coherent (sub) SI unit $10^{-7} \mathrm{~mol} \cdot \mathrm{J}^{-1}$ affords very similar $G$ values (within $4 \%$ ) as those in $(100 \mathrm{eV})^{-1}$, as $(100 \mathrm{eV})^{-1}=$ $1.036 \times 10^{-7} \mathrm{~mol} \cdot \mathrm{J}^{-1}$.

(44) SI unit of $g(x)$ is $\mathrm{m}^{-3} \cdot \mathrm{s}^{-1}$ for $E_{\mathrm{p}}$ in photon number basis and $\mathrm{mol} \cdot \mathrm{m}^{-3} \cdot \mathrm{s}^{-1}$ for $E_{n, \mathrm{p}}$ in chemical amount basis.

(45) Inverse of the lifetime of free carriers in the bulk of the crystalline particle.

(46) Superscript 0 (zero) indicates incident radiant power and refl reflected radiant power.

(47) Ratio of the photonic efficiency of a chemical process to that of a standard process, under otherwise identical conditions. At a constant photon flux, $\xi_{\text {rel }}$ is the ratio of initial reaction rates.

(48) The scattering coefficients should be carefully calculated independent of the absorption coefficient. The subscript $\lambda$ is omitted and the respective unit is not taken into account. The wavelength should be specified, e.g., $\sigma(300 \mathrm{~nm})$.

(49) The steradian is an SI supplementary unit, but is dimensionless, so has SI unit 1. 
(50) Common unit is $\mathrm{cm}^{2} \cdot \mathrm{g}^{-1} \cdot \mathrm{nm}^{-1}$. Absorption coefficient is always a spectral property; the subscript $\lambda$ may be omitted. The wavelength should be specified, e.g., $\alpha(300 \mathrm{~nm})$. Mass concentration is $\rho=m / V$.

(51) The specific napierian attenuance coefficient reduces to specific napierian absorption coefficient for a non-reflecting or scattering sample. The subscript $\lambda$ is omitted and the respective unit is not taken into account. The wavelength should be specified, e.g., $\beta(300 \mathrm{~nm})$. Mass concentration is $\rho=m / V$.

(52) Commonly expressed in $\mathrm{cm}^{2} \cdot \mathrm{g}^{-1}$.

(53) Other physical quantities $X$ such as irradiance, photon flux, photon fluence, photon fluence rate and radiant intensity may be used to derive the corresponding "spectral" quantity (relative to wavelength) by $X_{\lambda}=\mathrm{d} X / \mathrm{d} \lambda$. Analogous quantities relative to frequency or to wavenumber may also be defined. $[X]$ indicates the unit of the quantity $X[9]$.

(54) Common unit is $\mathrm{W} \cdot \mathrm{m}^{-2} \cdot \mathrm{nm}^{-1}$. Analogous quantities relative to frequency or to wavenumber may also be defined.

(55) Common unit is $\mathrm{s}^{-1} \cdot \mathrm{m}^{-2} \cdot \mathrm{sr}^{-1} \cdot \mathrm{nm}^{-1}$. This quantity can be expressed on a chemical amount basis by dividing $L_{\mathrm{p}, \Omega, \lambda}$ by the Avogadro constant. The name then is "spectral photon radiance, chemical amount basis". SI unit is $\mathrm{mol} \cdot \mathrm{s}^{-1} \cdot \mathrm{m}^{-3} \cdot \mathrm{sr}^{-1}$; common unit is einstein $\cdot \mathrm{s}^{-1} \cdot \mathrm{m}^{-2} \cdot \mathrm{sr}^{-1} \cdot \mathrm{nm}^{-1}$. Analogous quantities relative to frequency or to wavenumber may also be defined.

(56) Common unit is $\mathrm{W} \cdot \mathrm{m}^{-2} \cdot \mathrm{sr}^{-1} \cdot \mathrm{nm}^{-1}$. Analogous quantity relative to frequency or to wavenumber may also be defined.

(57) Common unit is $\mathrm{J} \cdot \mathrm{nm}^{-1}$. Analogous quantity relative to frequency or to wavenumber may also be defined.

(58) Common unit is $\mathrm{W} \cdot \mathrm{nm}^{-1}$. Analogous quantity relative to frequency or to wavenumber may also be defined.

(59) Relative biological or chemical photoresponse per incident radiant power as a function of wavelength.

(60) Common unit is $\mathrm{s}^{-1}$ (active site) $)^{-1}$.

(61) Also called the area turnover frequency.

\section{MEMBERSHIP OF SPONSORING BODIES}

Membership of the IUPAC Physical and Biophysical Chemistry Division Committee for the period 2010-2011 is as follows:

President: A. J. McQuillan (New Zealand); Vice President: K. Yamanouchi (Japan); Secretary: R. Marquardt (France); Past President: M. J. Rossi (Switzerland); Titular Members: J. H. Dymond (UK); A. Friedler (Israel); R. Guidelli (Italy); J.-G. Hou (China); B. D. Sykes (Canada); A. K. Wilson (USA); Associate Members: V. Barone (Italy); K. Bartik (Belgium); A. R. H. Goodwin (USA); V. Mišković-Stanković (Serbia); G. R. Moore (UK); M. Rico (Spain); National Representatives: K. Bhattacharyya (India); S.-J. Kim (Korea); V. Yu. Kukushkin (Russia); A. J. Mahmood (Bangladesh); O. V. Mamchenko (Ukraine); A. W. Mombrú Rodríguez (Uruguay); F. H. Quina (Brazil); N. Soon (Malaysia); V. Tsakova (Bulgaria); M. Witko (Poland).

Membership of the IUPAC Organic and Biomolecular Chemistry Division Committee for the period 2010-2011 is as follows:

President: G. J. Koomen (Netherlands); Vice President: K. N. Ganesh (India); Secretary: M. J. Garson (Australia); Past President: P. R. Tundo (Italy); Titular Members: N. A. Al-Awadi (Kuwait); M. A. Brimble (New Zealand); S. Fukuzumi (Japan); A. Griesbeck (Germany); C.-C. Liao (Taiwan); F. E. McDonald (USA); Associate Members: A. Brandi (Italy); M. C. Cesa (USA); M. I. Choudhary (Pakistan); B. Han (China); T. M. Krygowski (Poland); N. E. Nifantiev (Russia); National 
Representatives: V. Dimitrov (Bulgaria); J. A. R. Hasanen (Egypt); Md. A. Hashem (Bangladesh); H. Jacobs (Jamaica); S. H. Kang (Korea); G. Seoane (Uruguay); D. M. Sladić (Serbia); E. Uggerud (Norway); R. Villalonga Santana (Spain); Z. Zakarria (Malaysia).

The working party also included: D. Bahnemann (Institut für Technische Chemie, University of Hannover, Hannover, Germany); J. R. Bolton (Bolton Photosciences Inc., Edmonton, AB, Canada); L. K. Koopal (Laboratory of Physical Chemistry and Colloid Science, Wageningen University, Wageningen, The Netherlands); V. K. Ryabchuk (V.A. Fock Institute of Physics, St. Petersburg State University, St. Petersburg, Russia); E. Savinov (Boreskov Institute of Catalysis, Novosibirsk, Russia) ${ }^{\dagger}$.

In addition to the members of the working party, the following scientists collaborated with this Glossary: O. M. Alfano, M. Anpo, V. Augugliaro, C. Bohne, S. Esplugas, E. Oliveros, C. von Sonntag, R. G. Weiss, M. Schiavello ${ }^{\dagger}$.

\section{REFERENCES}

1. K. I. Zamaraev. In Studies in Surface Science and Catalysis, Vol. 101, part A, J. W. Hightower, W. N. Delgass, E. Iglesia, A. T. Bell (Eds.), p. 35, Elsevier, Amsterdam (1996).

2. R. K. Clayton. Photosynthesis, Cambridge University Press, Cambridge, UK (1980).

3. N. Serpone, A. V. Emeline. Int. J. Photoenergy 4, 91 (2002).

4. N. Serpone, A. Salinaro. Pure Appl. Chem. 71, 303 (1999).

5. V. N. Parmon. Catalysis Today 39, 137 (1997).

6. C. Minero, D. Vione. Appl. Catal., B 67, 257 (2006).

7. S. E. Braslavsky. Pure Appl. Chem. 79, 293 (2007).

8. IUPAC. Compendium of Chemical Terminology, $2^{\text {nd }}$ ed. (the "Gold Book"). Compiled by A. D. McNaught and A. Wilkinson. Blackwell Scientific Publications, Oxford (1997). XML on-line corrected version: doi:10.1351/goldbook (2006-) created by M. Nic, J. Jirat, B. Kosata; updates compiled by A. Jenkins.

9. IUPAC. Quantities, Units and Symbols in Physical Chemistry, $3^{\text {rd }}$ ed. (the "Green Book"). Prepared for publication by E. R. Cohen, T. Cvitaš, J. G. Frey, B. Holmström, K. Kuchitsu, R. Marquardt, I. Mills, F. Pavese, M. Quack, J. Stohner, H. L. Strauss, M. Takami, A. J. Thor, RSC Publishing, Cambridge, UK (2007).

10. V. N. Parmon, Y. I. Aristov. In Radiation Catalytic Processes in Dispersed Media, V. N. Parmon (Ed.), p. 6, Nauka, Novosibirsk (1992) (in Russian).

11. (a) V. Gold. Pure Appl. Chem. 55, 1281 (1983); see also (b) P. Müller. Pure Appl. Chem. 66, 1077 (1994).

12. International Commission on Illumination (CIE). International Lighting Vocabulary, Geneva, Switzerland (1987).

13. R. L. Burwell. Pure Appl. Chem. 46, 71 (1976).

14. M. Boudart, G. Djega-Mariadassou. Kinetics of Heterogeneous Catalytic Reactions, p. 6, Princeton University Press, Princeton (1984).

15. H. J. Kuhn, S. E. Braslavsky, R. Schmidt. Pure Appl. Chem. 76, 2105 (2004).

16. (a) A. E. Cassano, O. M. Alfano. Catalysis Today 58, 167 (2000); (b) R. J. Brandi, O. M. Alfano, A. E. Cassano. Environ. Sci. Technol. 34, 2623 (2000); (c) R. J. Brandi, O. M. Alfano, A. E. Cassano. Environ. Sci. Technol. 34, 2631 (2000).

17. (a) V. Augugliaro, L. Palmisano, M. Schiavello. AIChE J. 37, 1096 (1991); (b) V. Augugliaro, V. Loddo, L. Palmisano, M. Schiavello. In Photochemie Konzepte, Methoden, Experimente, D. Wöhrle, M. W. Tausch, W. D. Stohrer (Eds.), pp. 459-465, Wiley-VCH, Weinheim (1999).

${ }^{\dagger}$ Deceased. 
18. L. Bürgi, H. Sirringhaus, R. H. Friend. Appl. Phys. Lett. 80, 2913 (2002).

19. J. Barber. Chem. Soc. Rev. 38, 185 (2009).

20. D. Gust, T. A. Moore, A. L. Moore. Acc. Chem. Res. 34, 40 (2001).

21. A. J. Bard, R. Memming, B. Miller. Pure Appl. Chem. 63, 569 (1991).

22. J. D. Livingston. Electronic Properties of Engineering Materials, Wiley-VCH, Weinheim (1999).

23. K. Brennan. Introduction to Semiconductor Devices, Cambridge University Press, Cambridge, UK (2005).

24. A. V. Emeline, X. Zhang, M. Jin, T. Murakami, A. Fujishima. J. Phys. Chem. B 110, 7409 (2006).

25. S. Brunauer, P. H. Emmett, E. Teller. J. Am. Chem. Soc. 60, 309 (1938).

26. See, e.g., (a) D. Hufschmidt, D. Bahnemann, J. J. Testa, C. A. Emilio, M. I. Litter. J. Photochem. Photobiol., A 148, 223 (2002); (b) Y. H. Hsien, C. F. Chang, Y. H. Chen, S. F. Cheng. Appl. Catal., $B$ 31, 241 (2001).

27. R. Coekelbergs, A. Crucq, J. Decot, L. Degols, M. Randoux, L. Timmerman. J. Phys. Chem. Solids 26, 1983 (1965).

28. A. Emeline, G. Kataeva, A. Panasuk, V. Ryabchuk, N. Sheremetyeva, N. Serpone. J. Phys. Chem. $B$ 109, 5175 (2005).

29. M.-A. White. Properties of Materials, Oxford University Press, Oxford (1999).

30. A. J. Bard, L. R. Faulkner. Electrochemical Methods. Fundamentals and Applications, John Wiley, New York (1980).

31. <http://www.iupac.org/reports/periodic_table/index.html>.

32. A. V. Emeline, S. Polikhova, N. Andreev, V. Ryabchuk, N. Serpone. J. Phys. Chem. B 106, 5956 (2002).

33. H. P. Wagner, V. R. Gangilenka, A. DeSilva, H. Schmitzer, R. Scholz, T. U. Kampen. Phys. Rev. $B$ 73, 125323 (2006).

34. L. Smart, E. A. Moore. Solid State Chemistry: An Introduction, $3^{\text {rd }}$ ed., CRC Press, Taylor \& Francis, Boca Raton (2005).

35. V. Ryabchuk. Int. J. Photoenergy 6, 95 (2004).

36. R. Bonneau, J. Wirz, A. D. Zuberbühler. Pure Appl. Chem. 69, 979 (1997).

37. (a) J. G. Kirkwood. J. Chem. Phys. 2, 351 (1934); (b) C. J. F. Böttcher. Theory of Electrical Polarization, p. 145, Elsevier, Amsterdam (1973).

38. P. Wardman. J. Phys. Chem. Ref. Data 18, 1637 (1989).

39. G. J. Kavarnos. Fundamentals of Photoinduced Electron Transfer, VCH, New York (1993).

40. M. Pharr, G. Humphreys. In Physically Based Rendering: From Theory to Implementation, Series of Interactive 3D Technology, M. Kaufmann (Ed.), Elsevier (2004).

41. A. E. Cassano, C. A. Martin, R. J. Brandi, O. M. Alfano. Ind. Eng. Chem. Res. 34, 2155 (1995).

42. M. A. Fox, M. T. Dulay. Chem. Rev. 93, 341 (1993).

43. A. V. Emeline, V. K. Ryabchuk, N. Serpone. J. Phys. Chem. B 109, 18515 (2005).

44. S. A. Polikhova, N. S. Andreev, A. V. Emeline, V. K. Ryabchuk, N. Serpone. J. Phys. Chem. B 108, 2354 (2004).

45. M. L. Satuf, R. J. Brandi, A. E. Cassano, O. M. Alfano. Ind. Eng. Chem. Res. 44, 6643 (2005).

46. C. Kormann, D. W. Bahnemann, M. R. Hoffmann. Environ. Sci. Technol. 25, 494 (1991).

47. L. L. Basov, Y. K. Dolgikh, L. K. Polzik, Y. P. Solonitsyn. React. Kinet. Catal. Lett. 21, 277 (1982).

48. J. Chen, C.-S. Poon. Building Environ. 44, 1899 (2009).

49. M. Szklarczyk, J. O’M. Bockris. Appl. Phys. Lett. 42, 1035 (1963).

50. J. O’M. Bockris, Z. S. Minevski. Electrochim. Acta 39, 1471 (1994).

51. M. K. Nazeeruddin, F. De Angelis, S. Fantacci, A. Selloni, G. Viscardi, P. Liska, S. Ito, B. Takeru, M. Grätzel. J. Am. Chem. Soc. 127, 16835 (2005).

52. A. J. Nozik. Faraday Discuss. Chem. Soc. 70, 7 (1980). 
53. (a) A. J. Bard, M. Stratmann. Encyclopedia of Electrochemistry, 6, Semiconductor Electrodes and Photoelectrochemistry, S. Licht (Ed.), John Wiley, New York (2002); (b) <http://nsl.caltech.edu/ ressemiphoto.html>.

54. R. Van Grieken, M. De Bruin. Pure Appl. Chem. 66, 2513 (1994).

55. IUPAC. Chapter 16 in Compendium of Analytical Nomenclature, $3^{\text {rd }}$ ed. (the "Orange Book"). Prepared for publication by J. Inczédy, T. Lengyel, A. M. Ure, Blackwell Science, Oxford (1998). $<\mathrm{http}: / /$ media.iupac.org/publications/analytical_compendium/>.

56. T. Watanabe, T. Takizawa, K. Honda. J. Phys. Chem. 81, 1845 (1977).

57. M. B. Rubin, S. E. Braslavsky. Photochem. Photobiol. Sci. 9, 670 (2010).

58. ISO 31-6:1992(E). Handbook on Quantities and Units, Part 6: Light and Related Electromagnetic Radiations, International Organization for Standardization (ISO), Geneva, Switzerland (1992).

59. C. von Sonntag. The Chemical Basis of Radiation Biology, Taylor \& Francis, London (1987).

60. C. von Sonntag. Free-Radical-Induced DNA Damage and its Repair. A Chemical Perspective, Springer Verlag, Berlin (2006).

61. M. N. Ozisic. Radiative Transfer and Interaction with Conduction and Convection, John Wiley, New York (1973).

62. D. T. Sawyer, J. S. Valentine. Acc. Chem. Res. 14, 393 (1981).

63. P. R. Gogate, A. B. Pandit. AIChE J. 50, 1051 (2004).

64. Y. P. Solonitsyn, G. N. Kuzmin, A. L. Shurygin, V. M. Yurkin. Kinet. Catal. 17, 1092 (1976). Note the error in eq. 2 (in the English translation), defining the specific capacity $\theta_{\infty}$. It should correctly be: $\theta_{\infty}=Q_{\infty} /\left(m S_{0}\right)$, with $m$ the mass of adsorbent, $S_{0}$ the specific surface (called $a^{\mathrm{s}}$ in this Glossary) and $Q_{\infty}$ the maximum number of molecules adsorbed at infinite irradiation time.

65. A. V. Emeline, N. V. Sheremetyeva, N. V. Khomchenko, G. N. Kuzmin, V. K. Ryabchuk, W. Y. Teoh, R. Amal. J. Phys. Chem. C 113, 4566 (2009).

66. K. S. W. Sing, D. H. Everett, R. A. W. Haul, L. Moscou, R. A. Pierotti, J. Rouquerol, T. Siemieniewska. Pure Appl. Chem. 57, 603 (1985).

67. K. Demeestere, J. Dewulf, H. Van Langenhove. Crit. Rev. Environ. Sci. Technol. 37, 489 (2007).

68. J. W. Verhoeven. Pure Appl. Chem. 68, 2223 (1996).

69. <http://physics.nist.gov/cuu/Constants/index.html>.

Republication or reproduction of this report or its storage and/or dissemination by electronic means is permitted without the need for formal IUPAC permission on condition that an acknowledgment, with full reference to the source, along with use of the copyright symbol $\odot$, the name IUPAC, and the year of publication, are prominently visible. Publication of a translation into another language is subject to the additional condition of prior approval from the relevant IUPAC National Adhering Organization. 
Pure Appl. Chem., Vol. 83, No. 5, p. 1215, 2011.

doi:10.1351/PAC-REC-09-09-36_errata

(c) 2011 IUPAC: Publication date (Web): 13 April 2011

\section{Errata}

Glossary of terms used in photocatalysis and radiation catalysis (IUPAC Recommendations 2011). (S. E. Braslavsky, A. M. Braun, A. E. Cassano, A. V. Emeline, M. I. Litter, L. Palmisano, V. N. Parmon, N. Serpone). Pure Appl. Chem. 83, 931-1014 (2011); doi:10.1351/PAC-REC-09-09-36.

The following errors appeared in the Recommendations cited above:

p. 948: Entry einstein: in the first line below the entry, "mol" should be changed to "mole".

p. 955: Entry Gibbs energy of photoinduced electron transfer: in the second and third equations, "/J" should be removed from the left-hand side.

p. 995: Entry turnover frequency: in the note 3 equation, the numerator " $\mathrm{d} N$ " should be changed to " $\mathrm{d}(N / V)$ ".

p. 1001: Table entry number density of entities, number concentration: the symbol should be " $C$ " and the definition should be " $C=N / V$ ".

p. 1006: Table entry specific surface area: in the definition, " $a^{\mathrm{s}}=S / \mathrm{m}$ " should be changed to " $a \mathrm{~s}=S / m$ ".

p. 1008: Table entry turnover frequency in homogeneous catalysis: the numerator " $\mathrm{d} N$ " should be changed to " $\mathrm{d}(N / V)$ ".

p. 1008: Table entry turnover number: "TON" should be replaced with "(TON)". 